February 2006

hep-th/0602093

\title{
Quantization of models with non-compact quantum group symmetry. Modular XXZ magnet and lattice sinh-Gordon model.
}

\author{
ANDREI G. BYTSKo ${ }^{1}$ and JÖRG TESChNER ${ }^{2}$ \\ ${ }^{1}$ Steklov Mathematics Institute, Fontanka 27, 191023, St. Petersburg, Russia \\ ${ }^{2}$ DESY Theory Group, Notkestrasse 85, D-22603, Hamburg, Germany
}

\begin{abstract}
We define and study certain integrable lattice models with non-compact quantum group symmetry (the modular double of $\mathcal{U}_{q}(\mathfrak{s l}(2, \mathbb{R}))$ ) including an integrable lattice regularization of the sinh-Gordon model and a non-compact version of the XXZ model. Their fundamental Rmatrices are constructed in terms of the non-compact quantum dilogarithm. Our choice of the quantum group representations naturally ensures self-adjointness of the Hamiltonian and the higher integrals of motion. These models are studied with the help of the separation of variables method. We show that the spectral problem for the integrals of motion can be reformulated as the problem to determine a subset among the solutions to certain finite difference equations (Baxter equation and quantum Wronskian equation) which is characterized by suitable analytic and asymptotic properties. A key technical tool is the so-called Q-operator, for which we give an explicit construction. Our results allow us to establish some connections to related results and conjectures on the sinh-Gordon theory in continuous space-time. Our approach also sheds some light on the relations between massive and massless models (in particular, the sinh-Gordon and Liouville theories) from the point of view of their integrable structures.
\end{abstract}

\section{Contents}

$1 \quad$ Introduction 3

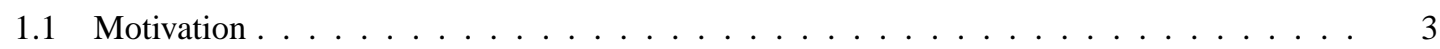

1.2 Lattice $\operatorname{sinh-Gordon}$ and the modular XXZ magnet . . . . . . . . . . . . . . 4

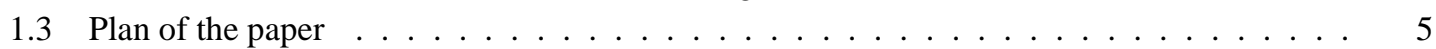

2 Modular XXZ magnet 6

2.1 Ouantum group symmetry $\mathcal{U}_{\alpha}(\mathfrak{s} \mathfrak{l}(2, \mathbb{R})) \ldots \ldots \ldots \ldots$

2.2 Representations $\mathcal{P}_{c}-$ algebra of observables $\ldots \ldots \ldots \ldots \ldots$

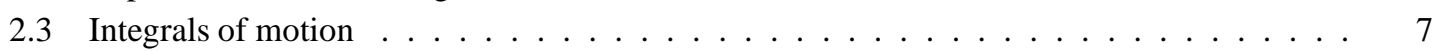

2.4 Fundamental R-operator and Hamiltonian . . . . . . . . . . . . . . . . . . . 8

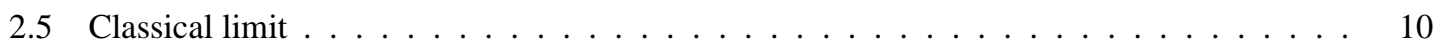

2.6 Comparison with similar models $\ldots \ldots \ldots \ldots \ldots \ldots$

\footnotetext{
${ }^{1}$ Supported in part by a Humboldt Stiftung fellowship, INTAS grant YS-03-55-962, the Russian Foundation for Fundamental Research grant 05-01-00922, and a grant from the Russian Science Support Foundation

${ }^{2}$ Part of this work was supported by a DFG Heisenberg fellowship
} 
\begin{tabular}{lll}
\hline Lattice sinh-Gordon model & 11
\end{tabular}

3.1 Definition of the model . . . . . . . . . . . . . . . . . . . . . . . 11

3.2 Fundamental R-operator and Hamiltonian . . . . . . . . . . . . . . . . . . . . . . 11

3.3 Relation with the continuum theory . . . . . . . . . . . . . . . . . . . 13

3.4 Failure of the algebraic Bethe ansatz $\ldots \ldots \ldots \ldots \ldots$

\begin{tabular}{|lll}
4 & O-operator and Baxter equation & 15
\end{tabular}

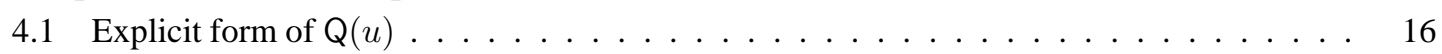

4.2 Analvtic properties of eigenvalues of $\mathrm{Q}(u) \ldots \ldots \ldots \ldots \ldots \ldots \ldots$

4.3 Self-duality and quantum Wronskian relation $\ldots \ldots \ldots \ldots$

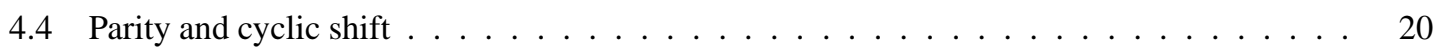

5 Separation of variables $\quad 21$

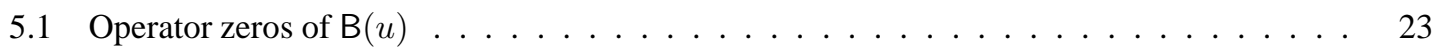

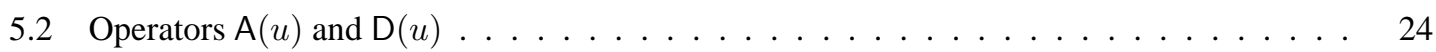

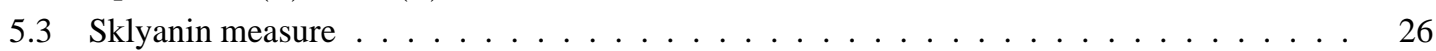

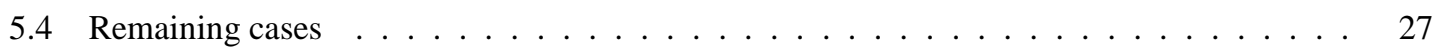

6 Concluding remarks - outlook 29

6.1 On the Baxter equations . . . . . . . . . . . . . . . . . . . . . . . . . . . . . 29

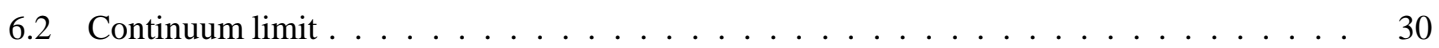

6.3 Connection with lattice Liouville model . . . . . . . . . . . . . . . . . 32

\begin{tabular}{|l|l|}
\hline A Special functions & 34 \\
\hline
\end{tabular}

A.1 Double Gamma function . . . . . . . . . . . . . . . . . . . . . . . . . . . . . . . . .

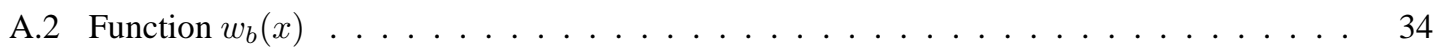

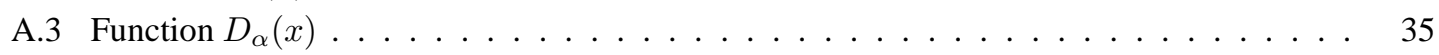

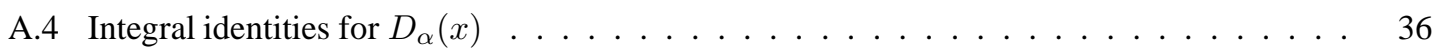

B Positivity versus self-duality of the representations $\mathcal{P}$

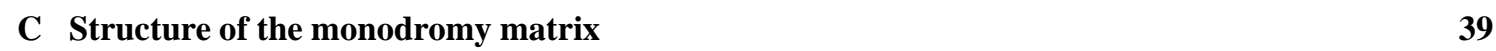

C.1 Expansions in the spectral parameter . . . . . . . . . . . . . . . . . . . . . 39

C.2 Ouantum determinant . . . . . . . . . . . . . . . . . . . . . 41

D Construction of the fundamental R-operator $\mathrm{R}(u)$

D.1 Clebsch-Gordan maps . . . . . . . . . . . . . . . . . . . . . . 44

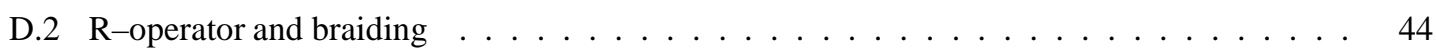

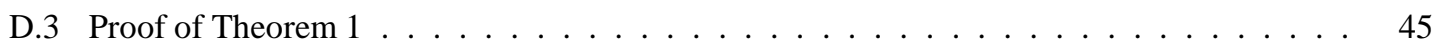

D.4 Clebsch-Gordan and Racah-Wigner coefficients for $\mathcal{P}$. . . . . . . . . . . . . . . . 48

D.5 Proof of Proposition $4 \ldots \ldots \ldots$. . . . . . . . . . . . . . . . 49

E Construction of the O-operator $Q(u)$

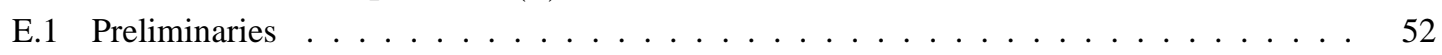

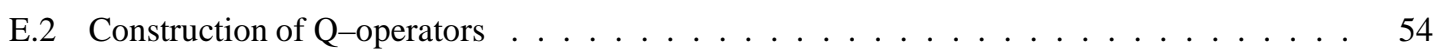

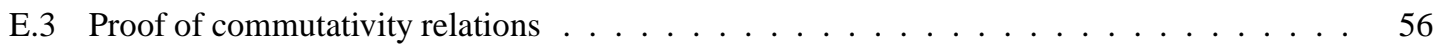




\section{Introduction}

\subsection{Motivation}

One of the main motivations for studying integrable lattice models is their role as a lattice regularization of quantum field theories in continuous space-time. Integrable nonlinear sigma models are of particular interest, and recently there has been a growing interest in nonlinear sigma models with non-compact target spaces. This interest is motivated by possible applications to string theory on curved space-times in general, and to gauge theories via the AdS-CFT correspondence in particular.

However, the quantization and the solution of such non-compact nonlinear sigma models still represents a major challenge for the field of integrable models. Compared to the better understood nonlinear sigma models with compact target spaces one may expect important qualitative differences, which make it problematic to apply the known techniques from the compact cases to the sigma models with non-compact target spaces. This point is exemplified by the relation between the Wess-Zumino-Novikov-Witten (WZNW) models associated to compact and non-compact symmetric spaces respectively. The solution of the latter is possible [T1], but it is considerably more difficult than the solution of WZNW models associated to compact groups.

In more general sigma models one can not hope to find the powerful Kac-Moody symmetries of the WZNW models but the integrable structure may still survive. In order to enter the next level of complexity one may therefore try to exploit the integrability of some of these models. Turning to a new class of models it is always advisable to look for the simplest member which still exhibits most of the new qualitative features. In the case of the conformal WZNW models it has turned out that Liouville theory already displays many of the relevant differences which distinguish the noncompact WZNW models from rational conformal field theories [T2]. Moving outside of the class of sigma models soluble thanks to Kac-Moody or similarly powerful chiral symmetries it seems natural to look for a useful counterpart of Liouville theory within this larger class of models.

A natural candidate for such a model exists: the sinh-Gordon model. Indeed, there is some evidence $[\mathrm{ZZ}] \mathrm{Lu}]$ that the sinh-Gordon model can be seen as a "deformation" of Liouville theory which preserves its integrable structure when the conformal symmetry is lost. While there certainly exists a good basis for the study of the sinh-Gordon model in infinite volume $-\mathrm{S}$-matrix and the form factors are known [VG $\mathrm{FMS}$ KMu, BL, Le] and the basic ingredients of the QISM approach were developed [S1] - there does not seem to exist a sytematic approach to the quantization and solution of the sinh-Gordon model in finite spatial volume yet. Part of the problem is due to the usual divergencies and ordering problems of quantum (field) theory. But the other part of the problem seems to be closely related to the non-compactness of the target space in the sinh-Gordon model.

Our main motivation behind the present project was therefore to find an integrable lattice regularization for the sinh-Gordon model. This not only tames the usual short distance singularities, it will also allow us to take care of the troubles from non-compactness of the target space in a mathematically well-defined framework. One particular feature that directly follows from the non-compactness of the target space will be the failure of the usual algebraic Bethe ansatz method [F1] for the model at hand. This failure means that we will have to use the more general separation of variables method [S2, S3, Sm1] instead. 


\subsection{Lattice sinh-Gordon and the modular XXZ magnet}

A quantum integrable system is a quantum system $(\mathcal{H}, \mathcal{A}, \mathrm{H})$, with Hilbert space $\mathcal{H}$, algebra of observables $\mathcal{A}$, Hamiltonian $\mathrm{H}$, in which there exists a set $\mathcal{Q}=\left\{\mathrm{T}_{0}, \mathrm{~T}_{1}, \ldots\right\}$ of self-adjoint operators such that

$$
\begin{aligned}
& \text { (A) }\left[\mathrm{T}, \mathrm{T}^{\prime}\right]=0 \quad \forall \mathrm{T}, \mathrm{T}^{\prime} \in \mathcal{Q}, \\
& \text { (B) }[\mathrm{T}, \mathrm{H}]=0 \quad \forall \mathrm{T} \in \mathcal{Q}, \\
& \text { (C) if }[\mathrm{T}, \mathrm{O}]=0 \text { for all } \mathrm{T} \in \mathcal{Q}, \text { then } \mathrm{O}=\mathrm{O}(\mathcal{Q}) \text {. }
\end{aligned}
$$

Property (C) expresses completeness of the set $\mathcal{Q}$ of integrals of motion. It is equivalent to the statement that the spectrum of $\mathcal{Q}$ is non-degenerate, i.e., that simultaneous eigenstates of $\mathrm{T}_{k}$, $k \in \mathbb{Z}^{\geq 0}$ are uniquely determined by the tuple of their eigenvalues. We will consider the so-called one-dimensional lattice models for which one has

$$
\mathcal{H}=\mathcal{K}^{\otimes \mathrm{N}}, \quad \mathcal{A}=\mathcal{B}^{\otimes \mathrm{N}},
$$

with one copy of Hilbert space $\mathcal{K}$ and algebra of local observables $\mathcal{B}$ being associated to each of the $\mathrm{N}$ sites of a one-dimensional lattice.

The quantum inverse scattering method (QISM) [FST, [F1] goes a long way towards the construction of large classes of quantum integrable models of this type. In this framework one usually characterizes $\mathcal{K}$ as a representation of a Hopf algebra $\mathfrak{U}$ of "symmetries", and $\mathcal{B}$ is generated from the operators which represent the elements of $\mathfrak{U}$ on $\mathcal{K}$. It is clear that the representation theoretic properties of $\mathcal{K}$ will influence the physical properties of the resulting integrable model decisively. Good control over these properties will be crucial in the construction and solution of such models.

In general it is a highly nontrivial problem to find the "right" representation $\mathcal{K}$ which leads to a useful lattice regularization of a particular quantum field theory. We will here propose a particular choice for $\mathcal{K}$ which will lead to a lattice model with particularly nice properties, and which will be shown to yield the sinh-Gordon Hamiltonian density in the continuum limit of the corresponding classical lattice model. The representations in question will be representations of the non-compact real form $\mathcal{U}_{q}(\mathfrak{s l}(2, \mathbb{R}))$ of $\mathcal{U}_{q}\left(\mathfrak{s l}_{2}\right)$ which have been studied in [PT1 [F3, PT2, BT].

The non-compactness of the target space will be reflected in the infinite-dimensionality of the representation $\mathcal{K}$. It is furthermore worth noting that the same representations were previously found to reflect a key internal structure of Liouville theory [PT1 T2]. In view of the existing evidence $[\mathrm{ZZ}, \overline{\mathrm{Lu}}]$ for the connection between Liouville theory and the sinh-Gordon model, it is quite natural that the same class of representations appears in our lattice version of the sinhGordon model as well.

The corresponding representations possess a remarkable duality - they are simultaneously representations of $\mathcal{U}_{q}\left(\mathfrak{s l}_{2}\right)$ and $\mathcal{U}_{\tilde{q}}\left(\mathfrak{s l}_{2}\right)$, where $q=e^{i \pi b^{2}}$ and $\tilde{q}=e^{i \pi b^{-2}}$. One may therefore view them [F3] as representations of the modular double $\mathcal{U}_{q}\left(\mathfrak{s l}_{2}\right) \otimes \mathcal{U}_{\tilde{q}}\left(\mathfrak{s l}_{2}\right)$ (see also [KLS, BT]). The parameter $b$ turns out to be proportional to the coupling constant $\beta$ of the sinh-Gordon model. The self-duality of our representations will be directly related to the self-duality of the sinh-Gordon model under $b \rightarrow b^{-1}$ which was previously observed in its scattering theory. The importance of this self-duality for our analysis can hardly be over-emphasized.

It turns out that there is a close relative of our lattice sinh-Gordon model which is simpler in some respects. This integrable lattice model can be seen as a non-compact counterpart of the 
XXZ model with spins in infinite-dimensional representations of the modular double. We will refer to this model as the modular XXZ magnet. As some technical issues are simpler in the case of the modular XXZ magnet, we will first construct the latter model before we turn to the lattice sinh-Gordon model. In any case, it seems to us that the study of the modular XXZ magnet is of interest in its own right. We note in particular that despite the different underlying representation theory, our model has many structural similarities with the non-compact XXX type magnet based on infinite-dimensional highest weight representations of $\mathfrak{s l}_{2}$ which was studied in [DKM, KM]. The latter model plays an important role in high energy QCD [Li, [FK1].

\subsection{Plan of the paper}

To make our paper accessible to a reasonably wide audience, we presented the general description of our approach, the main definitions and results in the main body of the paper and collected more technical developments in Appendices. The article is organized as follows.

In Section 2 we define the modular XXZ magnet in terms of the representations $\mathcal{P}_{s}$ describe its Hilbert space of states, derive the corresponding fundamental $\mathrm{R}$-operator $\mathrm{R}(u)$, and discuss the construction of its Hamiltonian and the set of integrals of motion $\mathcal{Q}$.

The same is done for the lattice sinh-Gordon model in Section 3. We show that the Hamiltonian density of the sinh-Gordon model is recovered in the continuum limit of the corresponding classical model. We also show that the algebraic Bethe ansatz fails due to the non-compactness of the target space in the sinh-Gordon model.

An important first step towards the solution of these models is taken in Section 4. We refine the spectral problem for the integrals of motion $\mathrm{T}_{k}$ by constructing the $\mathrm{Q}$-operator $\mathrm{Q}(u)$ which is related to the $\mathrm{T}_{k}$ via the so-called Baxter equation. Analyzing the properties of $\mathrm{Q}(u)$, we derive a set of conditions for its eigenvalues $q_{t}(u)$ which can be seen as quantization conditions and which replace the usual Bethe ansatz equations in our models. The self-duality of our representations furthermore allows us to derive the so-called quantum Wronskian relation for the sinh-Gordon model with odd $\mathrm{N}$, which encodes valuable additional information about the spectrum.

In order to show that the conditions found in Section 4 are also sufficient to characterize the spectrum we apply the separation of variables approach to our models in Section 5.

Section 6 contains concluding remarks on the conditions which characterize the spectrum of our models, the continuum limit, and the relation with the lattice and continuum versions of Liouville theory. We observe in particular that our results are consistent with the results and conjectures of $[\mathrm{Za}] \mathrm{Lu}]$ on the continuum sinh-Gordon model in a nontrivial way.

Appendices contain necessary technical details. Appendix A collects the relevant information on the special functions that we use. Appendix B discusses the precise mathematical nature of the self-duality $b \rightarrow b^{-1}$ of the representations that we use. Appendic $\mathrm{C}$ contains some important technical results on the structure of the monodromy matrix. Appendix D is devoted to the construction of the fundamental R-operator, the key object for the construction of local integrals of motion for the lattice models. Appendix E contains details on the construction of the Q-operator Q $(u)$.

Acknowledgements. We thank S. Derkachov, L. Faddeev, S. Lukyanov, N. Reshetikhin, and especially F. Smirnov for stimulating discussions. A.B. is grateful to J.T. and R. Schrader for hospitality during his visits to the Institute for Theoretical Physics, FU-Berlin and to V. Schomerus for hospitality during a visit to DESY, Hamburg. 


\section{Modular XXZ magnet}

In this section we will begin to develop the QISM for the modular XXZ magnet - an XXZ type non-compact spin chain, which has $\mathcal{U}_{q}(\mathfrak{s l}(2, \mathbb{R}))$ as a quantum symmetry.

\subsection{Quantum group symmetry $\mathcal{U}_{q}(\mathfrak{s t}(2, \mathbb{R}))$}

Let $q=e^{i \gamma}, \gamma=\pi b^{2}, b \in(0,1)$. We will also use the notation $Q=b+b^{-1}$.

The quantum group $\mathcal{U}_{q}\left(\mathfrak{s l}_{2}\right)$ is a Hopf algebra with generators $E, F, K, K^{-1}$ satisfying the relations

$$
K E=q E K, \quad K F=q^{-1} F K, \quad[E, F]=\frac{1}{q-q^{-1}}\left(K^{2}-K^{-2}\right)
$$

and equipped with the following co-product:

$$
\begin{aligned}
& \Delta(E)=E \otimes K+K^{-1} \otimes E, \\
& \Delta(F)=F \otimes K+K^{-1} \otimes F, \quad \Delta(K)=K \otimes K .
\end{aligned}
$$

The relevant real form of $\mathcal{U}_{q}\left(\mathfrak{s l}_{2}\right)$ is $\mathcal{U}_{q}(\mathfrak{s l}(2, \mathbb{R}))$, which is defined by the following star-structure:

$$
K^{*}=K, \quad E^{*}=E, \quad F^{*}=F .
$$

The center of $\mathcal{U}_{q}(\mathfrak{s l}(2, \mathbb{R}))$ is generated by the $q$-Casimir element:

$$
C=(2 \sin \gamma)^{2} F E-q K^{2}-q^{-1} K^{-2}+2, \quad C^{*}=C .
$$

\subsection{Representations $\mathcal{P}_{s}$ - algebra of observables}

A one-parameter family of unitary representations $\mathcal{P}_{s}$ of $\mathcal{U}_{q}(\mathfrak{s l}(2, \mathbb{R}))$ can be constructed from a pair of self-adjoint operators $\mathrm{p}$ and $\mathrm{x}$ on $L^{2}(\mathbb{R})$ which satisfy $[\mathrm{p}, \mathrm{x}]=(2 \pi i)^{-1}$ as follows:

$$
\begin{aligned}
& \pi_{s}(E) \equiv \mathrm{E}_{s}=e^{+\pi b \times} \frac{\cosh \pi b(\mathrm{p}-s)}{\sin \gamma} e^{+\pi b \times}, \\
& \pi_{s}(F) \equiv \mathrm{F}_{s}=e^{-\pi b \times} \frac{\cosh \pi b(\mathrm{p}+s)}{\sin \gamma} e^{-\pi b \times},
\end{aligned}
$$

For this representation

$$
\mathrm{C}_{s} \equiv \pi_{s}(C)=4 \cosh ^{2} \pi b s .
$$

It is remarkable and important that the operators $\mathrm{E}_{s}, \mathrm{~F}_{s}$ and $\mathrm{K}_{s}$ are positive self-adjoint. Indeed, the representations $\mathcal{P}_{s}$ are the only "reasonable" class of representations of $\mathcal{U}_{q}(\mathfrak{s l}(2, \mathbb{R}))$ which have this property. This property will play a key role in much of the following developments. It will in particular ensure seld-adjointness of operators such as the Hamiltonian and the integrals of motion. It is also the mathematical basis for the self-duality of the representations $\mathcal{P}_{s}$, as shown in Appendix B(see, in particular, eq. (B.5)).

The lattice model that we are about to define will have one of the representations $\mathcal{P}_{s}$ attached to each site of the one-dimensional lattice. This means that we take

$$
\mathcal{H}=\left(L^{2}(\mathbb{R})\right)^{\otimes \mathrm{N}}
$$


as the Hilbert space of our model, and let

$$
\widehat{\mathcal{A}}=\left(\pi_{s}(\mathcal{U})\right)^{\otimes \mathrm{N}}, \quad \mathcal{U} \equiv \mathcal{U}_{q}(\mathfrak{s l}(2, \mathbb{R}))
$$

be a set of generators for our algebra of observables. Note that the operators in $\widehat{\mathcal{A}}$ are all unbounded, but there exists a basis for $\widehat{\mathcal{A}}$ whose elements are positive self-adjoint (see Appendix $\mathbb{B}$ ). The latter fact allows us to construct large classes of non-polynomial operator functions of the generators in $\widehat{\mathcal{A}}$ via standard functional calculus for self-adjoint operators and/or pseudo-differential operator calculus.

\subsection{Integrals of motion}

As the next step we shall introduce our main ansatz for the set $\mathcal{Q}$ of integrals of motion using the usual scheme of the QISM. To this aim let us assemble the generators of $\widehat{\mathcal{A}}$ into the following L-matrix acting on $\mathbb{C}^{2} \otimes \mathcal{P}_{s}$ :

$$
L^{\mathrm{xxz}}(u)=\left(\begin{array}{cc}
e^{\pi b u} \mathrm{k}_{s}-e^{-\pi b u} \mathrm{k}_{s}^{-1} & i e^{\pi b u} \mathrm{f}_{s} \\
i e^{-\pi b u} \mathrm{e}_{s} & e^{\pi b u} \mathrm{k}_{s}^{-1}-e^{-\pi b u} \mathrm{k}_{s}
\end{array}\right), \quad u \in \mathbb{C} .
$$

In the definition of $L^{\mathrm{xxz}}(u)$ we have used the rescaled generators $\mathrm{e}_{s}, \mathrm{f}_{s}, \mathrm{k}_{s}$ which are defined by

$$
\mathrm{e}_{s}=(2 \sin \gamma) \mathrm{E}_{s}, \quad \mathrm{f}_{s}=(2 \sin \gamma) \mathrm{F}_{s}, \quad \mathrm{k}_{s}=\mathrm{K}_{s} .
$$

Occasionally we will omit the superscript xxz for the sake of brevity. The defining relations (2) and (3) of $\mathcal{U}_{q}(\mathfrak{s l}(2, \mathbb{R}))$ are equivalent to

$$
\begin{aligned}
R_{12}(u) L_{13}(u+v) L_{23}(v) & =L_{23}(v) L_{13}(u+v) R_{12}(u), \\
(\operatorname{id} \otimes \Delta) L^{ \pm} & =L_{13}^{ \pm} L_{12}^{ \pm},
\end{aligned}
$$

where $L^{ \pm}$arise in the decomposition

$$
L(u)=e^{\pi b u} L^{+}-e^{-\pi b u} L^{-},
$$

and the auxiliary R-matrix is given by

$$
R(u)=\left(\begin{array}{cccc}
\sinh \pi b(u+i b) & & & \\
& \sinh \pi b u & i \sin \pi b^{2} e^{\pi b u} & \\
& i \sin \pi b^{2} e^{-\pi b u} & \sinh \pi b u & \\
& & & \sinh \pi b(u+i b)
\end{array}\right) .
$$

Out of the L-matrices we may then construct the monodromy $\mathrm{M}(u)$,

$$
\mathrm{M}(u) \equiv\left(\begin{array}{ll}
\mathrm{A}(u) & \mathrm{B}(u) \\
\mathrm{C}(u) & \mathrm{D}(u)
\end{array}\right) \equiv L_{\mathrm{N}}(u) \cdot \ldots \cdot L_{2}(u) \cdot L_{1}(u) .
$$

Of particular importance is the one-parameter family of operators:

$$
\mathrm{T}(u)=\operatorname{tr}(\mathrm{M}(u))=\mathrm{A}(u)+\mathrm{D}(u) .
$$

The trace in 17 is taken over the auxiliary space, which is $\mathbb{C}^{2}$ for the models we consider. 
Lemma 1. The operators $\mathrm{T}_{m}$ which appear in the expansion

$$
\mathbf{T}(u)=e^{\pi b \mathrm{~N} u} \sum_{m=0}^{\mathrm{N}}\left(-e^{-2 \pi b u}\right)^{m} \mathrm{~T}_{m},
$$

are positive self-adjoint and mutually commuting, $\left[\mathrm{T}_{m}, \mathrm{~T}_{n}\right]=0$.

Commutativity of $\mathrm{T}_{m}$ follows by the standard argument from the relation (12); the proof of their positivity and self-adjointness is given in Appendix [C]

Definition 1. Let us define the set of commuting charges as $\mathcal{Q}=\left\{\mathrm{T}_{0}, \mathrm{~T}_{1}, \ldots, \mathrm{T}_{\mathrm{N}}\right\}$.

Remark 1. Self-adjointness of the operators $\mathrm{T}_{m}, m=0, \ldots, \mathrm{N}$ ensures the existence of a joint spectral decomposition for the family $\mathcal{Q}$.

Let us emphasize that the crucial positivity of the operators $T_{m}$ is a direct consequence of the fact that the generators $E, F$ and $K$ of $\mathcal{U}_{q}(\mathfrak{s l}(2, \mathbb{R}))$ are represented by positive operators in the representations $\mathcal{P}_{s}$. This makes clear why these representations are particularly well-suited for defining non-compact analogues of the XXZ spin chains. We will later make a similar observation in the lattice sinh-Gordon model.

\subsection{Fundamental R-operator and Hamiltonian}

Our next aim is to construct a local Hamiltonian which commutes with the elements of $\mathcal{Q}$. We will adapt the approach from [FTT] to the case at hand. The main ingredient of this approach is the so-called fundamental $\mathrm{R}$-operator corresponding to (10). This operator, $\mathrm{R}_{s_{2} s_{1}}^{\mathrm{XXz}}(u)$, acts on $\mathcal{P}_{s_{2}} \otimes \mathcal{P}_{s_{1}}$ and is supposed to satisfy the commutation relations

$$
\mathrm{R}_{23}(u) L_{13}(u+v) L_{12}(v)=L_{12}(v) L_{13}(u+v) \mathrm{R}_{23}(u) .
$$

For our purposes it will be sufficient ${ }^{1}$ to deal with $\mathrm{R}(u) \equiv \mathrm{R}_{s s}^{\mathrm{xxz}}(u)$ acting on $\mathcal{P}_{s} \otimes \mathcal{P}_{s}$.

Definition 2. Let the operator $\mathrm{R}(u)$ be defined by the formula

$$
\mathrm{R}(u)=\mathrm{P} w_{b}(u+\mathrm{s}) w_{b}(u-\mathrm{s})=\mathrm{P} D_{u}(\mathrm{~s}),
$$

where $\mathrm{P}$ is the operator which just permutes the two tensor factors in $\mathcal{P}_{s} \otimes \mathcal{P}_{s}$, and $\mathrm{s}$ is the unique positive self-adjoint operator such that

$$
4 \cosh ^{2} \pi b \mathrm{~s}=\left(\pi_{s} \otimes \pi_{s}\right) \Delta(C)
$$

The special functions $w_{b}(x)$ and $D_{\alpha}(x)$ are defined in Appendix $₫$

Theorem 1. The operator $\mathrm{R}(u)$ satisfies the equation (19) where $L(u)$ is given by (10).

The proof of this theorem is given in Appendix D where the construction of the operator $\mathrm{R}_{s_{2} s_{1}}^{\mathrm{XXz}}(u)$ is presented for the general case, $s_{1} \neq s_{2}$, see equation (D.14).

\footnotetext{
${ }^{1}$ The general solution $\mathrm{R}_{s_{2} s_{1}}^{\mathrm{XXz}}(u)$ is needed if we wish to construct an inhomogeneous spin chain, for instance the one with alternating spins (see, e.g., $|\mathrm{BD}|$ ).
} 
The operator $\mathrm{R}(u)$ has the following further properties

$$
\begin{aligned}
\text { regularity } & \mathrm{R}(0)=\mathrm{P}, \\
\text { reflection property } & \mathrm{R}(-u)=\mathrm{P} \mathrm{R}^{-1}(u) \mathrm{P}, \\
\text { unitarity } & \mathrm{R}^{*}(u)=\mathrm{R}^{-1}(u) \quad \text { for } u \in \mathbb{R},
\end{aligned}
$$

which follow from the properties of $w_{b}(u)$ and $D_{\alpha}(x)$ listed in Appendix $\mathrm{A}$

The regularity condition (22) allows us to apply the standard recipe [FTT] of the QISM in order to construct a Hamiltonian with local (nearest neighbour) interaction of sites:

$$
\begin{aligned}
\mathrm{H}^{\mathrm{xxz}} & =\frac{i}{\pi b} \mathrm{U}^{-1}\left[\frac{\partial}{\partial u} \operatorname{tr}_{a}\left(\mathrm{R}_{a \mathrm{~N}}(u) \cdot \ldots \cdot \mathrm{R}_{a 2}(u) \cdot \mathrm{R}_{a 1}(u)\right)\right]_{u=0} \\
& =\left.\sum_{n=1}^{\mathrm{N}} \frac{i}{\pi b} \partial_{u} D_{u}\left(\mathrm{~s}_{n, n+1}\right)\right|_{u=0}=\sum_{n=1}^{\mathrm{N}} H_{n, n+1}^{\mathrm{xxz}} .
\end{aligned}
$$

We are using the following notation: We identify $\mathrm{s}_{\mathrm{N}, \mathrm{N}+1} \equiv \mathrm{s}_{\mathrm{N}, 1}$, the cyclic shift operator $\mathrm{U}$ is defined by $\mathrm{U} f\left(x_{1}, x_{2}, \ldots, x_{\mathrm{N}}\right)=f\left(x_{2}, \ldots, x_{\mathrm{N}}, x_{1}\right)$, and the subscript $a$ stands for an auxiliary copy of the space $\mathcal{P}_{s}$. The trace operation is defined for an operator $\mathrm{O}: \mathcal{P}_{s} \mapsto \mathcal{P}_{s}$ in the usual way: if the integral kernel of $\mathrm{O}$ in the momentum representation is given by $O\left(k \mid k^{\prime}\right)$, then $\operatorname{tr} \mathrm{O}=$ $\int_{-\infty}^{\infty} d k O(k \mid k)$. According to this definition we have $\operatorname{tr}_{a} \mathrm{P}_{a b}=1_{b}$.

Substituting the integral representation (A.20) for $D_{u}(x)$ into 25), we obtain the following local Hamiltonian density

$$
H_{n, n+1}^{\mathrm{xxz}}=-\frac{1}{\pi} \int_{\mathbb{R}+i 0} d t \frac{\cos \left(2 b t \mathrm{~s}_{n, n+1}\right)}{\sinh t \sinh b^{2} t} .
$$

It may then be shown in the usual manner [FTT] that $\mathrm{H}$ commutes with the trace of the monodromy matrix, $\mathrm{T}(u)$, which means that

$$
\left[\mathrm{H}, \mathrm{T}_{k}\right]=0, \text { for } k=0, \ldots, \mathrm{N}
$$

As in any quantum mechanical system, the fundamental problem that we would like to solve is the problem to determine the spectrum of $\mathrm{H}$. However, thanks to the commutativity (27) it seems promising to first solve the following

Auxiliary Spectral Problem: Find the spectrum of the operator $\mathrm{T}(u)$, i.e., the joint spectral decomposition for the family of operators $\mathcal{Q}=\left\{\mathrm{T}_{0}, \ldots, \mathrm{T}_{\mathrm{N}}\right\}$.

Simple counting of the degrees of freedom suggests that the spectrum of $\mathcal{Q}$ may be simple, i.e., that an eigenstate $\Psi_{t}$ of $\mathrm{T}(u)$,

$$
\mathrm{T}(u) \Psi_{t}=t(u) \Psi_{t}
$$

is uniquely characterized by the eigenvalue $t(u)=e^{\pi b \mathrm{~N} u} \sum_{m=0}^{\mathrm{N}}\left(-e^{-2 \pi b u}\right)^{m} t_{m}$. This would imply that $\mathrm{H}=\mathrm{H}(\mathcal{Q})$, so that the solution to the Auxiliary Spectral Problem also yields the spectral decomposition of $\mathrm{H}$. 


\subsection{Classical limit}

Let us discuss the classical limit of the quantum Hamiltonian (26). So far we have been working in the units where the Planck constant $\hbar$ was chosen to be unity. In order to recover it explicitly, we have to make the following rescaling

$$
b^{2} \rightarrow \hbar b^{2}, \quad \mathrm{p} \rightarrow \hbar^{-\frac{1}{2}} \mathrm{p}, \quad \mathrm{x} \rightarrow \hbar^{-\frac{1}{2}} \mathrm{x}, \quad \mathrm{s} \rightarrow \hbar^{-\frac{1}{2}} \mathrm{~s}, \quad s \rightarrow \hbar^{-\frac{1}{2}} s,
$$

so that we have $q=e^{i \hbar \gamma}, \gamma=\pi b^{2}$. The operators $\mathrm{e}_{s}, \mathrm{f}_{s}, \mathrm{k}_{s}$ are not affected by the procedure (28). In the limit $\hbar \rightarrow 0$ they become classical variables e, $\mathrm{f}, \mathrm{k}$ with the following Poisson brackets obtained by the correspondence principle, $[,] \rightarrow-i \hbar\{$,

$$
\{e, k\}=\gamma k e, \quad\{f, k\}=-\gamma k f, \quad 2 \gamma\{e, f\}=k^{2}-k^{-2} .
$$

Using the asymptotics (computed by means of contour integration)

$$
\begin{aligned}
\lim _{\hbar \rightarrow 0} \frac{\hbar}{2} \int_{\mathbb{R}+i 0} d t \frac{e^{-i t z}}{\sinh t \sinh \hbar t} & =\frac{1}{2} \int_{\mathbb{R}+i 0} d t \frac{e^{-i t z}}{t \sinh t} \\
& =\sum_{n \geq 1}^{\infty} \frac{(-1)^{n} e^{\pi n z}}{n}=-\log \left(1+e^{\pi z}\right)
\end{aligned}
$$

we obtain from (26) the corresponding classical lattice Hamiltonian density,

$$
\begin{aligned}
& H_{n, n+1}^{\mathrm{xxz}, \mathrm{cl}}= \lim _{h \rightarrow 0} \hbar H_{n, n+1}^{\mathrm{xxz}}=\frac{1}{\gamma} \log \left(4 \cosh ^{2} \pi b \mathrm{~s}_{n, n+1}^{\mathrm{cl}}\right)=\frac{1}{\gamma} \log \left(\mathrm{C}_{n, n+1}^{\mathrm{cl}}\right) \\
&=\frac{1}{\gamma} \log \left(\left(\mathrm{e}_{n} \mathrm{f}_{n+1}+\mathrm{f}_{n} \mathrm{e}_{n+1}\right) \mathrm{k}_{n}^{-1} \mathrm{k}_{n+1}\right. \\
&\left.\quad+2 \mathrm{k}_{n}^{-2} \mathrm{k}_{n+1}^{2}+2 \cosh (2 \pi b s)\left(\mathrm{k}_{n}^{-2}+\mathrm{k}_{n+1}^{2}\right)+2\right) .
\end{aligned}
$$

Here $C_{n, n+1}^{c l}$ is the classical limit of the tensor Casimir operator given by (21).

\subsection{Comparison with similar models}

L-matrix (10) and R-matrix (15) are suitable for the usual XXZ model as well. The only (but essential) difference is that in the latter case matrix coefficients of the L-matrix act on a highest weight module of $\mathcal{U}_{q}\left(\mathfrak{s l}_{2}\right)$. We also remark that (10) differs from the most commonly used "standard" L-matrix in that it contains extra factors $e^{ \pm \pi b u}$ in the off-diagonal elements. The "standard" L-matrix does not satisfy (14) but is symmetric (if the matrix transposition ${ }^{T}$ is combined with the operator transposition ${ }^{t}$ such that $\mathrm{f}_{s}^{t}=\mathrm{e}_{s}$ and $\mathrm{k}_{s}^{t}=\mathrm{k}_{s}$ ) and corresponds to the symmetric auxiliary R-matrix (34).

Let $\psi_{b}(x)$ denote the logarithmic derivative of the function $S_{b}(x)$ defined by A.15. Properties A.16-A.18 show that $S_{b}(x)$ can be regarded as a b-analogue of the gamma function. The Hamiltonian density (26) rewritten in terms of $\psi_{b}(x)$ looks as follows

$$
H_{n, n+1}^{\mathrm{xxz}}=\frac{2}{\pi b} \psi_{b}\left(\frac{Q}{2}+i \mathrm{~s}\right)=\frac{2}{\pi b} \psi_{b}\left(\frac{Q}{2}-i \mathrm{~s}\right)=\frac{1}{\pi b}\left(\psi_{b}\left(\frac{Q}{2}+i \mathrm{~s}\right)+\psi_{b}\left(\frac{Q}{2}-i \mathrm{~s}\right)\right) .
$$

Equivalence of these expressions is due to A.18. The last of them resembles the form of the Hamiltonian density of the non-compact XXX magnet [DKM] expressed in terms of the ordinary $\psi$-function. 
The special function $w_{b}(u)$ is closely related (cf. Eq. (D.7) to the non-compact quantum dilogarithm $g_{b}(u)$. Counterparts of (20) and (D.14) for the compact XXZ magnet look similar in terms of the q-gamma function which, in turn, is closely related to the compact analogue of $g_{b}(u)$ given by $s_{q}(t)=\prod_{n=0}^{\infty}\left(1+t q^{2 n+1}\right)$.

It is also worth noticing that the $\mathrm{R}$-operator (20) resembles the fundamental $\mathrm{R}$-operator $r(\mathrm{~s}, \lambda)$ found in [FV] for a simpler L-matrix related to the Volterra model [V1]. The main difference is that the operator argument $\mathrm{s}$ of $r(\mathrm{~s}, \lambda)$ has a much simpler structure in terms of the variables $\mathrm{p}$ and $x$. It would be interesting to clarify the connection between these two R-operators.

\section{Lattice sinh-Gordon model}

\subsection{Definition of the model}

In this section we will begin to develop the QISM for a lattice version of the sinh-Gordon model, which has $\mathcal{U}_{q}(\mathfrak{s l}(2, \mathbb{R}))$ as a quantum symmetry. We are going to keep much of the set-up from Section 2] but we will now be using the following L-matrix acting on $\mathbb{C}^{2} \otimes \mathcal{P}_{s}$

$$
L^{\mathrm{SG}}(u)=\frac{1}{i} e^{-\pi b s}\left(\begin{array}{cc}
i \mathrm{e}_{s} & e^{\pi b u} \mathrm{k}_{s}^{-1}-e^{-\pi b u} \mathrm{k}_{s} \\
e^{\pi b u} \mathrm{k}_{s}-e^{-\pi b u} \mathrm{k}_{s}^{-1} & i \mathrm{f}_{s}
\end{array}\right) .
$$

L-matrix (33) satisfies the intertwining relation (12) where the auxiliary R-matrix is now given by

$$
R(u)=\left(\begin{array}{cccc}
\sinh \pi b(u+i b) & & & \\
& \sinh \pi b u & i \sin \gamma & \\
& i \sin \gamma & \sinh \pi b u & \\
& & & \sinh \pi b(u+i b)
\end{array}\right)
$$

This R-matrix possesses the following symmetry

$$
\left[R(u), \sigma_{a} \otimes \sigma_{a}\right]=0,
$$

where $\sigma_{a}, a=1,2,3$, are the Pauli matrices. We may then proceed the same way as in Section 2 to define the operator $\mathrm{T}(u)$ and the family $\mathcal{Q}=\left\{\mathrm{T}_{0}, \ldots, \mathrm{T}_{\mathrm{N}}\right\}$ of commuting observables. We again find (see Appendix C) that the corresponding operators $\mathrm{T}_{m}, m=0, \ldots, \mathrm{N}$ are positive self-adjoint as a direct consequence of the positivity of $\mathrm{e}_{s}, \mathrm{f}_{s}, \mathrm{k}_{s}$. The existence of a joint spectral decomposition for the family $\mathcal{Q}$ is thereby ensured by the spectral theorem (cf. Remark 1 ).

\subsection{Fundamental R-operator and Hamiltonian}

Now our aim is to find the fundamental $\mathrm{R}$-operator $\mathrm{R}_{s_{2} s_{1}}^{\mathrm{SG}}(u)$ corresponding to $\mathrm{L}$-matrix (33). Fortunately, it turns out that it can be constructed from the R-operator of the XXZ chain in a simple way. To demonstrate this, we first introduce an automorphism $\theta$ such that

$$
\theta(\mathrm{p})=-\mathrm{p}, \quad \theta(\mathrm{x})=-\mathrm{x} .
$$

It is useful to notice that $\theta$ can be realzied as an inner automorphism, $\theta(\mathrm{O})=\Omega \mathrm{O} \Omega^{-1}$, where $\Omega$ is the parity operator whose action in the momentum representation is defined by $(\Omega f)(k)=f(-k)$. 
Notice that $\Omega$ is unitary and satisfies $\Omega^{-1}=\Omega$. Observe that for the representation $\mathcal{P}_{s}$ we have (cf. [6)

$$
\theta(\mathrm{e})=\mathrm{f}, \quad \theta(\mathrm{f})=\mathrm{e}, \quad \theta(\mathrm{k})=\mathrm{k}^{-1} .
$$

Definition 3. Let the operator $\mathrm{R}_{s_{2} s_{1}}^{\mathrm{SG}}(u)$ be defined by the formula

$$
\mathrm{R}_{s_{2} s_{1}}^{\mathrm{SG}}(u)=(\mathrm{k} \otimes \mathrm{k})^{-i \frac{u}{2 b}} \cdot(\Omega \otimes 1) \cdot \mathrm{R}_{s_{2} s_{1}}^{\mathrm{XXZ}}(u) \cdot(1 \otimes \Omega) \cdot(\mathrm{k} \otimes \mathrm{k})^{-i \frac{u}{2 b}},
$$

where $\mathrm{R}_{s_{2} s_{1}}^{\mathrm{Xxz}}(u)$ is given by (20) if $s_{1}=s_{2}$ and by (D.14) otherwise.

Proposition 1. The operator $\mathrm{R}_{s_{2} s_{1}}^{\mathrm{SG}}(u)$ satisfies the equation (19) where $L(u)$ is given by (33).

Proof. It will be convenient to consider $\check{\mathrm{R}}_{12}^{\mathrm{SG}}(u) \equiv \mathrm{P}_{21} \mathrm{R}_{s_{2} s_{1}}^{\mathrm{SG}}(u): \mathcal{P}_{s_{2}} \otimes \mathcal{P}_{s_{1}} \rightarrow \mathcal{P}_{s_{1}} \otimes \mathcal{P}_{s_{2}}$ and analogously defined $\check{\mathrm{R}}_{12}^{\mathrm{XXz}}(u) \equiv \mathrm{P}_{21} \mathrm{R}_{s_{2} s_{1}}^{\mathrm{XXz}}(u)$. Equation (19) for $\mathrm{R}_{s_{2} s_{1}}^{\mathrm{SG}}(u)$ is then equivalent to

$$
\check{\mathrm{R}}_{23}^{\mathrm{SG}}(u) L_{13}^{\mathrm{SG}}(u+v) L_{12}^{\mathrm{SG}}(v)=L_{13}^{\mathrm{SG}}(v) L_{12}^{\mathrm{SG}}(u+v) \check{\mathrm{R}}_{23}^{\mathrm{SG}}(u) .
$$

Let us also note that, by using $(\mathrm{k} \otimes \mathrm{k})^{-1} \check{\mathrm{R}}_{12}^{\mathrm{xxz}}(u)(\mathrm{k} \otimes \mathrm{k})=\check{\mathrm{R}}_{12}^{\mathrm{xxz}}(u)$, we may rewrite the expression for $\check{\mathrm{R}}_{12}^{\mathrm{SG}}(u)$ which follows from 38 as

$$
\begin{aligned}
& \check{\mathrm{R}}_{12}^{\mathrm{SG}}(u)=(\mathrm{id} \otimes \theta)\left(\check{\mathrm{R}}_{12}^{\prime}(u)\right), \\
& \check{\mathrm{R}}_{12}^{\prime}(u) \equiv(\mathrm{k} \otimes 1)^{-i \frac{u}{b}} \cdot \check{\mathrm{R}}_{12}^{\mathrm{XXz}}(u) \cdot(1 \otimes \mathrm{k})^{i \frac{u}{b}} .
\end{aligned}
$$

The key to the proof of the Proposition will then be the following relation between the L-matrices $L^{\mathrm{SG}}(u)$ and $L^{\mathrm{XXZ}}(u)$

$$
L^{\mathrm{SG}}(u)=-i e^{-\pi b s} \sigma_{1} \mathrm{k}^{-i \frac{u}{b}} L^{\mathrm{xxz}}(u) \mathrm{k}^{i \frac{u}{b}} \equiv \sigma_{1} L^{\prime}(u) .
$$

Inserting (41) into (39), we get an expression which contains $\sigma_{1} L_{13}^{\prime}(u) \sigma_{1}$. Observe that (cf. (37)

$$
\sigma_{1} L^{\prime}(u) \sigma_{1}=(i d \otimes \theta) L^{\prime}(u) .
$$

Therefore, by using $\theta\left(\mathrm{O}_{1} \mathrm{O}_{2}\right)=\theta\left(\mathrm{O}_{1}\right) \theta\left(\mathrm{O}_{2}\right)$, one finds that 39 is equivalent to

$$
\check{\mathrm{R}}_{23}^{\prime}(u) L_{13}^{\prime}(u+v) L_{12}^{\prime}(v)=L_{13}^{\prime}(v) L_{12}^{\prime}(u+v) \check{\mathrm{R}}_{23}^{\prime}(u),
$$

which is now easily reduced to the Theorem 1 (its general case for $\mathrm{R}_{s_{2} s_{1}}^{\mathrm{XXz}}(u)$ ).

It is easy to see that properties (22)-(24) of the R-operator of the modular XXZ magnet hold for $\mathrm{R}(u) \equiv \mathrm{R}_{s s}^{\mathrm{SG}}(u)$ as well. Therefore, using the regularity of $\mathrm{R}(u)$, we can construct a Hamiltonian with nearest neighbour interaction of sites by using the same recipe that we used to derive (25). This yields

$$
\begin{aligned}
H_{n, n+1}^{\mathrm{SG}} & =\left.\frac{i}{\pi b} \partial_{u} \check{\mathrm{R}}_{n, n+1}^{\mathrm{SG}}(u)\right|_{u=0} \stackrel{40}{=}(i d \otimes \theta) H_{n, n+1}^{\mathrm{xxz}}+\frac{1}{\gamma} \log \left(\mathrm{k}_{n} \mathrm{k}_{n+1}\right) \\
& =-\frac{1}{\pi} \int_{\mathbb{R}+i 0} d t \frac{\cos \left(2 b t \hat{\mathbf{s}}_{n, n+1}\right)}{\sinh t \sinh b^{2} t}+\frac{1}{\gamma} \log \left(\mathrm{k}_{n} \mathrm{k}_{n+1}\right)
\end{aligned}
$$

where $\hat{\mathrm{s}}=(1 \otimes \Omega) \mathrm{s}(1 \otimes \Omega)$ is the unique positive self-adjoined operator on $\mathcal{P}_{s} \otimes \mathcal{P}_{s}$ such that

$$
4 \cosh ^{2} \pi b \hat{\mathrm{s}}=\left(\pi_{s} \otimes\left(\theta \circ \pi_{s}\right)\right) \Delta(C) .
$$




\subsection{Relation with the continuum theory}

To begin with, we may first compute the classical limit, $\hbar \rightarrow 0$, of (44) in the same way as we derived (31). Using (37), we obtain

$$
\begin{aligned}
H_{n, n+1}^{\mathrm{SG}, \mathrm{cl}}= & \frac{1}{\gamma} \log \left(\mathrm{e}_{n} \mathrm{e}_{n+1}+\mathrm{f}_{n} \mathrm{f}_{n+1}+2\left(\mathrm{k}_{n}^{-1} \mathrm{k}_{n+1}^{-1}+\mathrm{k}_{n} \mathrm{k}_{n+1}\right)\right. \\
& \left.+2 \cosh (2 \pi b s)\left(\mathrm{k}_{n}^{-1} \mathrm{k}_{n+1}+\mathrm{k}_{n} \mathrm{k}_{n+1}^{-1}\right)\right) .
\end{aligned}
$$

In order to establish the relation with the sinh-Gordon model it will be convenient to change variables as follows:

$$
2 \pi b \mathrm{p}_{n}=-\beta \Phi_{n}, \quad 4 \pi b \mathrm{x}_{n}=\beta\left(\frac{1}{2} \Pi_{n}-\Phi_{n}\right), \quad \beta=b \sqrt{8 \pi} .
$$

The variables $\Phi_{n}$ and $\Pi_{n}$ will then turn out to correspond to the (discretized) sinh-Gordon field and its conjugate momentum, respectively. The classical field and momentum variables defined in (47) satisfy the Poisson-bracket relations $\left\{\Pi_{n}, \Phi_{m}\right\}=\delta_{n m}$. The Hamiltonian (46) now looks as follows

$$
\begin{aligned}
H_{n, n+1}^{\mathrm{SG}, \mathrm{cl}} & =\frac{1}{\gamma} \log \frac{4}{\mu}\left(\frac{1}{2} \cosh \frac{\beta}{4}\left(\Pi_{n}+\Pi_{n+1}\right)+\frac{1+\mu^{2}}{2} \cosh \frac{\beta}{2}\left(\Phi_{n}-\Phi_{n+1}\right)\right. \\
& +\frac{\mu}{2} \cosh \beta\left(\Phi_{n}-\frac{1}{4}\left(\Pi_{n}+\Pi_{n+1}\right)\right)+\frac{\mu}{2} \cosh \beta\left(\Phi_{n+1}-\frac{1}{4}\left(\Pi_{n}+\Pi_{n+1}\right)\right) \\
& \left.+\mu \cosh \frac{\beta}{2}\left(\Phi_{n}+\Phi_{n+1}\right)+\frac{\mu^{2}}{4} \cosh \beta\left(\Phi_{n}+\Phi_{n+1}-\frac{1}{4}\left(\Pi_{n}+\Pi_{n+1}\right)\right)\right)
\end{aligned}
$$

where $\mu=e^{-2 \pi b s}$. In order to define the relevant limit leading to the continuous sinh-Gordon model, let us combine the limit of vanishing lattice spacing $\mathrm{N} \rightarrow \infty, \Delta \rightarrow 0(R=\mathrm{N} \Delta / 2 \pi$ is kept fixed) with the limit where the representation parameter $s$ goes to infinity in such a way that the mass parameter $m$ defined via

$$
\frac{1}{4} m \Delta=e^{-\pi b s} .
$$

stays finite. In addition we shall assume the standard correspondence between lattice and continuous variables:

$$
\Pi_{n} \rightarrow \Pi(x) \Delta, \quad \Phi_{n} \rightarrow \Phi(x), \quad x=n \Delta .
$$

We then find the following limiting expression for the Hamiltonian density:

$$
\sum_{n} \frac{1}{\Delta} H_{n, n+1}^{\mathrm{SG}, \mathrm{cl}} \rightarrow \mathrm{const}+\int_{0}^{2 \pi R} d x\left(\frac{1}{2} \Pi^{2}+\frac{1}{2}\left(\partial_{x} \Phi\right)^{2}+\frac{m^{2}}{\beta^{2}} \cosh \beta \Phi\right)
$$

thus recovering the continuous sinh-Gordon model.

It is also instructive to see what happens to the L-matrix in this limit. In the classical continuous limit, i.e., when $m$ in (49) is kept fixed and $\hbar, \Delta \rightarrow 0$, Eqs. (28) and (50) show that L-matrix (33) becomes

$$
L^{\mathrm{SG}}\left(\frac{u}{\pi b}\right) \rightarrow\left(\begin{array}{ll}
1 & 0 \\
0 & 1
\end{array}\right)+\Delta U^{\mathrm{SG}}(u)+O\left(\Delta^{2}\right),
$$

where $U^{\mathrm{SG}}(u)$ is the well-known $U$-matrix from the Lax pair for the classical continuous sinhGordon model [KBI, S1],

$$
U^{\mathrm{SG}}(u)=\left(\begin{array}{cc}
\frac{\beta}{4} \Pi(x) & \frac{m}{2 i} \sinh \left(u-\frac{\beta}{2} \Phi(x)\right) \\
\frac{m}{2 i} \sinh \left(u+\frac{\beta}{2} \Phi(x)\right) & -\frac{\beta}{4} \Pi(x)
\end{array}\right) .
$$


Remark 2. The classical lattice Hamiltonian density (46) resembles that found in [Ta] for the lattice sine-Gordon model. However, relation between the quantum Hamiltonians is less clear because the fundamental $\mathrm{R}$-operator proposed in [Ta] is represented as a product of $\mathrm{R}$-operators of the type $r(\mathrm{~s}, \lambda)$ which we mentioned at the end of Subsection 2.6 Possibly, recent results on factorization of R-operators [DKK] will help to clarify the connection between our construction and that used in [Ta, FV]

\subsection{Failure of the algebraic Bethe ansatz}

For the sake of clarity it may be worthwhile explaining in some detail why the algebraic Bethe ansatz is not suitable for the solution of the lattice sinh-Gordon model.

To begin with, let us observe that the L-matrix (33) has no pseudo-vacuum state, i.e., a vector $\Psi$ such that $L_{21}^{\mathrm{SG}}(u) \Psi=0$. Indeed, this would require that $\mathrm{k}_{n} \Psi=0=\mathrm{k}_{n}^{-1} \Psi$ for all $n=1, \ldots, \mathrm{N}$. Such a vector does not exist.

For the sine-Gordon model, one circumvents this difficulty by considering the composite L-matrix, $\mathcal{L}(u)$, which is product of two L-matrices [FST] [K]. For $\gamma=\pi \frac{m}{n}, n, m \in \mathbb{N}$, exponential operators $e^{i \beta \Phi_{n}}, e^{i \beta \Pi_{n}}$ admit finite-dimensional representations. In this case there exists a vector $\Psi$ that is annihilated by $\mathcal{L}_{21}(u)$. This makes it possible to apply the algebraic Bethe ansatz technique. Let us therefore consider the analogous construction for the sinh-Gordon model. Let $\mathcal{L}^{\mathrm{SG}}(u)=L_{2}^{\mathrm{SG}}(u) L_{1}^{\mathrm{SG}}(u+\varpi)$, where we introduced the shift by the constant $\varpi \in \mathbb{R}$ in order to increase the generality of our consideration. We then have

$$
\mathcal{L}_{21}^{\mathrm{SG}}(u)=i e^{\pi b u}\left(\mathrm{k} \otimes \mathrm{e}+e^{\pi b \varpi} \mathrm{f} \otimes \mathrm{k}\right)-i e^{-\pi b u}\left(\mathrm{k}^{-1} \otimes \mathrm{e}+e^{-\pi b \varpi} \mathrm{f} \otimes \mathrm{k}^{-1}\right) .
$$

The requirement that a vector $\Psi$ is annihilated by $\mathcal{L}_{21}^{\mathrm{SG}}(u)$ is equivalent to the two equations

$$
\left(\mathrm{k}^{ \pm 1} \otimes \mathrm{e}+e^{ \pm \pi b \varpi} \mathrm{f} \otimes \mathrm{k}^{ \pm 1}\right) \Psi=0 .
$$

We claim that there does not exist a reasonable (even in the distributional sense) state $\Psi$ with such properties. Indeed, note that

$$
\mathrm{k} \otimes \mathrm{e}+e^{\pi b \varpi} \mathrm{f} \otimes \mathrm{k}=\left(\mathrm{k}^{i \frac{\varpi}{b}} \Omega \otimes 1\right) \cdot\left(\mathrm{k}^{-1} \otimes \mathrm{e}+\mathrm{e} \otimes \mathrm{k}\right) \cdot\left(\mathrm{k}^{i \frac{\varpi}{b}} \Omega \otimes 1\right)^{-1}
$$

where $\Omega$ is the parity operation introduced in Subsection 3.2 The operator $\left(k^{i \frac{w}{b}} \Omega \otimes 1\right)$ is unitary, which allows us to conclude that $\mathrm{k} \otimes \mathrm{e}+e^{\pi b \varpi} \mathrm{f} \otimes \mathrm{k}$ and $\mathrm{k}^{-1} \otimes \mathrm{e}+\mathrm{e} \otimes \mathrm{k}$ have the same spectrum. However, the latter operator represents $(2 \sin \gamma) \Delta(E)$ on $\mathcal{P}_{s} \otimes \mathcal{P}_{s}$ (cf. eq. (3)). Unitarity of the Clebsch-Gordan maps (see Appendix D.1) implies that this operator has the same spectrum as $\mathrm{e}_{s}$. The unitary transformation used in the proof of Lemma 4 in Appendix B maps $\mathrm{e}_{s}$ to $e^{2 \pi b \mathrm{x}}$. It is now clear that all these operators do not have an eigenfunction with eigenvalue zero, as would be necessary to construct a Bethe vacuum.

Remark 3. Keeping in mind that the sinh-Gordon variables are just linear combinations of $\mathrm{p}$ and $\mathrm{x}$, cf. (47), we now see quite clearly that the failure of the Bethe ansatz is connected with the fact that the target space (the space in which the fields take their values) is non-compact. We expect this to be a general lesson. 


\section{Q-operator and Baxter equation}

As an important first step towards the solution of the Auxiliary Spectral Problem we shall now find necessary conditions for a function $t(u)$ to be eigenvalue of the operator $\mathrm{T}(u)$. In order to do this we are going to construct an operator $\mathrm{Q}(u)$ which satisfies the following properties:

(i) $\mathrm{Q}(u)$ is a normal operator, $\mathrm{Q}(u) \mathrm{Q}^{*}(v)=\mathrm{Q}^{*}(v) \mathrm{Q}(u)$,

(ii) $\mathrm{Q}(u) \mathrm{Q}(v)=\mathrm{Q}(v) \mathrm{Q}(u)$,

(iii) $\mathrm{Q}(u) \mathrm{T}(u)=\mathrm{T}(u) \mathrm{Q}(u)$,

(iv) $\mathrm{Q}(u) \mathrm{T}(u)=(a(u))^{\mathrm{N}} \mathrm{Q}(u-i b)+(d(u))^{\mathrm{N}} \mathrm{Q}(u+i b)$.

The first and the second property imply that all operators $\mathrm{Q}(u), u \in \mathbb{C}$ can be simultaneously diagonalized and their eigenvectors form a complete systems of states in the Hilbert space. The third and the fourth property imply that $\mathrm{T}(u)$ will be diagonal whenever $\mathrm{Q}(u)$ is. One may therefore consider the spectral problem for $\mathrm{Q}(u)$ as a refinement of the spectral problem for $\mathrm{T}(u)$.

Let us now consider an eigenstate $\Psi_{t}$ for $\mathrm{T}(u)$ with eigenvalue $t(u), \mathrm{T}(u) \Psi_{t}=t(u) \Psi_{t}$. Thanks to property (iii) above we may assume that it is an eigenstate for $\mathrm{Q}(u)$ as well,

$$
\mathrm{Q}(u) \Psi_{t}=q_{t}(u) \Psi_{t}
$$

It follows from property (iv) that the eigenvalue $q_{t}(u)$ must satisfy the so-called Baxter equation

$$
t(u) q_{t}(u)=(a(u))^{\mathrm{N}} q_{t}(u-i b)+(d(u))^{\mathrm{N}} q_{t}(u+i b) .
$$

We will construct the operator $\mathrm{Q}(u)$ explicitly — see Subsection 4.1 This will allow us to determine the analytic and - for the lattice sinh-Gordon model with N odd (the SGo-model) - the asymptotic properties that the eigenvalues $q_{t}(u)$ must have, namely

$$
\begin{aligned}
& \text { (i) } q_{t}(u) \text { is meromorphic in } \mathbb{C} \text {, with poles of maximal order } \mathrm{N} \text { in } \Upsilon_{-s} \cup \bar{\Upsilon}_{s}, \\
& \text { where } \Upsilon_{s}=\left\{s+i\left(\frac{Q}{2}+n b+m b^{-1}\right), \quad n, m \in \mathbb{Z}^{\geq 0}\right\}, \quad \bar{\Upsilon}_{s} \equiv\left(\Upsilon_{s}\right)^{*}, \\
& \text { (ii) } q_{t}^{\text {SGo }}(u) \sim\left\{\begin{array}{ll}
\exp \left(+\pi i \mathrm{~N}\left(s+\frac{i}{2} Q\right) u\right) \text { for }|u| \rightarrow \infty, & |\arg (u)|<\frac{\pi}{2}, \\
\exp \left(-\pi i \mathrm{~N}\left(s+\frac{i}{2} Q\right) u\right) \text { for }|u| \rightarrow \infty, & |\arg (u)|>\frac{\pi}{2} .
\end{array}\right]
\end{aligned}
$$

The derivation of these properties is discussed in Subsection 4.2. This means that there is the following necessary condition for a polynomial $t(u)$ to be eigenvalue of the operator $\mathrm{T}(u): t(u)$ can only be an eigenvalue of $\mathrm{T}(u)$ if there exists a meromorphic function $q_{t}(u)$ with singular behavior and asymptotic behavior given in (58) which is related to $t(u)$ by the Baxter equation (57).

The problem to classify the solutions to this condition is of course still rather nontrivial. However, previous experience from other integrable models suggests that the Baxter equation supplemented by the analytic and asymptotic properties 58 is indeed a useful starting point for the determination of the spectrum of the model, see also our Subsections 6.1 and 6.2 for some further remarks. We will discuss in the next section how the separation of variables method may allow us to show that the conditions above are also sufficient for $t(u)$ to be an eigenvalue of $\mathrm{T}(u)$.

Convention: We will use the superscripts $\mathrm{SG}$ and $\mathrm{xxz}$ to distinguish analogous operators within the two models we consider. However, we will simply omit these superscripts in any equation which holds in the two cases alike. 


\subsection{Explicit form of $Q(u)$}

Let us now describe explicitly the Q-operators for the models that we introduced in Sections 2 and 3 For this purpose we will work in the representation where the operators $\mathrm{x}_{r}, r=1, \ldots, \mathrm{N}$ are diagonal. This representation will be called the Schrödinger representation for the Hilbert space of a lattice model. Let $\mathbf{x} \equiv\left(x_{1}, \ldots, x_{\mathrm{N}}\right), \mathbf{x}^{\prime} \equiv\left(x_{1}^{\prime}, \ldots, x_{\mathrm{N}}^{\prime}\right)$. We will denote the integral kernel of the operator $\mathrm{Q}(u)$ in the Schrödinger representation by $Q_{u}\left(\mathbf{x}, \mathbf{x}^{\prime}\right)$. We will also use the following notations

$$
\sigma \equiv s+\frac{i}{2} Q, \quad \bar{\sigma} \equiv s-\frac{i}{2} Q,
$$

where $s$ stands for the spin of the representation $\mathcal{P}_{s}$.

Definition 4. Let the $Q$-operators $Q_{ \pm}^{b}(u), b=\mathrm{xxz}, \mathrm{sG}$, be defined in the Schrödinger representation by the following kernels

$$
\begin{aligned}
& Q_{+; u}^{b}\left(\mathbf{x}, \mathbf{x}^{\prime}\right)= \\
& =\left(D_{-s}(u)\right)^{\mathrm{N}} \prod_{r=1}^{\mathrm{N}} D_{\frac{1}{2}(\bar{\sigma}-u)}\left(x_{r}-x_{r}^{\prime}\right) D_{\frac{1}{2}(\bar{\sigma}+u)}\left(x_{r-1}-\varepsilon_{b} x_{r}^{\prime}\right) D_{-s}\left(x_{r}-\varepsilon_{b} x_{r-1}\right), \\
& Q_{-; u}^{b}\left(\mathbf{x}, \mathbf{x}^{\prime}\right)=\prod_{r=1}^{\mathrm{N}} D_{\frac{1}{2}(u-\sigma)}\left(x_{r}-x_{r}^{\prime}\right) D_{-\frac{1}{2}(u+\sigma)}\left(x_{r}-\varepsilon_{b} x_{r-1}^{\prime}\right) D_{s}\left(x_{r}^{\prime}-\varepsilon_{b} x_{r-1}^{\prime}\right),
\end{aligned}
$$

where $\varepsilon_{\mathrm{xxz}}=1, \varepsilon_{\mathrm{SG}}=-1$, and, in the sinh-Gordon case, $s$ is related to the parameters $m, \Delta$ as in (49).

Theorem 2. Let $\mathrm{T}^{\mathrm{b}}(u), b=\mathrm{xxz}, \mathrm{Sg}$ be the transfer-matrices corresponding to the L-matrices (10) and (33).

(i) The operators $Q_{ \pm}^{b}(u)$ satisfy all relations in (55).

(ii) The Baxter equation (55-iv) holds for $\mathrm{Q}_{ \pm}^{b}(u)$ with the following coefficients

$$
\begin{aligned}
a^{\mathrm{XXZ}}(u) & =2 \sinh \pi b(u-\sigma), \quad d^{\mathrm{XXz}}(u)=2 \sinh \pi b(u+\sigma), \\
d^{\mathrm{SG}}(u) & =a^{\mathrm{SG}}(-u)=e^{\pi b\left(u+i \frac{b}{2}\right)}+\left(\frac{m \Delta}{4}\right)^{2} e^{-\pi b\left(u+i \frac{b}{2}\right)}
\end{aligned}
$$

(iii) The operators $Q_{ \pm}^{b}(u)$ satisfy the relation

$$
\mathrm{Q}_{+}^{b}(u) \mathrm{Q}_{-}^{b}(v)=\mathrm{Q}_{-}^{b}(v) \mathrm{Q}_{+}^{b}(u) .
$$

The proof of this Theorem is given in Appendix $\mathrm{E}$ It is worth noting that $\mathrm{Q}_{-}^{b}(u)$ and $\mathrm{Q}_{+}^{b}(u)$ are related by hermitian conjugation as follows.

$$
\mathrm{Q}_{-}^{b}(u)=\left(D_{-s}(u)\right)^{\mathrm{N}}\left(\mathrm{Q}_{+}^{b}(\bar{u})\right)^{*} .
$$

This allows us to mostly focus on $\mathrm{Q}^{b}(u) \equiv \mathrm{Q}_{+}^{b}(u)$, but it is nevertheless sometimes useful to consider $\mathrm{Q}_{-}^{b}(u)$ as well. The corresponding eigenvalues $q_{t}^{ \pm}(u)$ are consequently related as

$$
q_{t}^{-}(u)=\left(D_{-s}(u)\right)^{\mathrm{N}} \overline{q_{t}^{+}(\bar{u})} .
$$

Relation (65) will imply that $q_{t}^{+}$and $q_{t}^{-}$have the same analytic and asymptotic properties (58). 
Remark 4. It is sometimes useful to observe (see Subsection E.2) that the operator $Q^{b}(u)$ can be factorized as follows:

$$
Q^{b}(u)=Y^{b}(u) \cdot Z,
$$

The operators $Y^{b}(u)$ and $Z$ in $(66)$ are represented by the kernels

$$
\begin{aligned}
Y_{u}^{\mathrm{b}}\left(\mathbf{x}, \mathbf{x}^{\prime}\right) & =\prod_{r=1}^{\mathrm{N}} D_{\frac{1}{2}(u-\sigma)}\left(x_{r}-\varepsilon_{b} x_{r+1}^{\prime}\right) D_{-\frac{1}{2}(u+\sigma)}\left(x_{r}-x_{r}^{\prime}\right), \\
Z\left(\mathbf{x}, \mathbf{x}^{\prime}\right) & =\left(w_{b}\left(i \frac{Q}{2}-2 s\right)\right)^{\mathrm{N}} \prod_{r=1}^{\mathrm{N}} D_{\bar{\sigma}}\left(x_{r}-x_{r}^{\prime}\right),
\end{aligned}
$$

respectively, where $\varepsilon_{\mathrm{xxz}}=1, \varepsilon_{\mathrm{SG}}=-1$.

Remark 5. The relations (55) do not define the Q-operator $\mathrm{Q}(u)$ uniquely. For instance, for a given $\mathrm{Q}(u)$, relations (55) are also fulfilled for $\mathrm{Q}^{\prime}(u)=(\varphi(u))^{\mathrm{N}} \mathrm{O} \mathrm{Q}(u)$, where $\varphi(u)$ is a scalar function and $\mathrm{O}$ is a unitary operator that commutes with $\mathrm{Q}(u)$ and $\mathrm{T}(u)$. The coefficients in Baxter equation (55-iv) for $\mathrm{Q}(u)$ and $\mathrm{Q}^{\prime}(u)$ are related via

$$
a^{\prime}(u)=\frac{\varphi(u)}{\varphi(u-i b)} a(u), \quad d^{\prime}(u)=\frac{\varphi(u)}{\varphi(u+i b)} d(u) .
$$

Thus, there is no canonical way to fix these coefficients. However, their combination $a(u) d(u-i b)$ remains invariant; its value is related to the quantum determinant if the latter can be defined for the L-matrix of the model in question (see Appendix C.2).

\subsection{Analytic properties of eigenvalues of $\mathrm{Q}(u)$}

We now turn to the derivation of the analytic properties of eigenvalues of $\mathrm{Q}(u)$. More precisely we shall prove the following:

\section{Theorem 3.}

(i) The operators $\mathrm{Q}_{ \pm}^{\mathrm{xxz}}(u)$ and $\mathrm{Q}_{ \pm}^{\mathrm{SG}}(u)$ are meromorphic functions of $u$ in $\mathbb{C}$ with poles of maximal order $\mathrm{N}$ contained in $\Upsilon_{-s} \cup \bar{\Upsilon}_{s}$.

(ii) Denote $\mathrm{Q}_{ \pm}^{\mathrm{SGo}}(u) \equiv \mathrm{Q}_{ \pm}^{\mathrm{SG}}(u)$ for $\mathrm{N}$ odd. This operator has the following asymptotic behavior

$$
\mathrm{Q}_{+}^{\mathrm{SGo}}(u) \sim \begin{cases}\mathrm{Q}_{+\infty} \exp (+i \pi \mathrm{N} \sigma u) & \text { for }|u| \rightarrow \infty,|\arg (u)|<\frac{\pi}{2}, \\ \mathrm{Q}_{-\infty} \exp (-i \pi \mathrm{N} \sigma u) & \text { for }|u| \rightarrow \infty,|\arg (u)|>\frac{\pi}{2},\end{cases}
$$

where $\mathrm{Q}_{ \pm \infty}$ are commuting unitary operators related to each other as follows

$$
Q_{-\infty}=\Omega Q_{+\infty}
$$

Here $\Omega$ is the parity operator (its action in the Schrödinger representation is given by (82)).

(iii) $\mathrm{Q}_{-}^{\mathrm{SGo}}(u)$ has asymptotic behavior of the same form (70) with $\mathrm{Q}_{ \pm \infty}$ replaced by $\mathrm{Q}_{ \pm \infty}^{*}$. 
Proof. In order to prove part (i) of the Theorem it clearly suffices to consider the corresponding statements for the eigenvalues $q_{t}^{ \pm}(u)$. Let us first explain why the properties of $q_{t}^{+}(u)$ and $q_{t}^{-}(u)$ described in the theorem are the same. Given that the theorem holds for $q_{t}^{+}(u)$, we infer that poles of $\overline{q_{t}^{+}(\bar{u})}$ are contained in $\Upsilon_{s} \cup \bar{\Upsilon}_{-s}$. But, since they are of maximal order $\mathrm{N}$, they cancel in 65) against the N-th order zeroes of $\left(D_{-s}(u)\right)^{\mathrm{N}}$ (see properties of $D_{\alpha}(x)$ in Appendix A.3). Thus, the only possible poles of $q_{t}^{-}(u)$ are those of $\left(D_{-s}(u)\right)^{\mathrm{N}}$, i.e., they are of maximal order $\mathrm{N}$ and contained in $\Upsilon_{-s} \cup \bar{\Upsilon}_{s}$.

The proof of part (i) will be exactly analogous for the cases of the XXZ magnet and the sinhGordon model. Only the latter case will therefore be discussed explicitly. We will study the equation (56), which is equivalent to

$$
\int_{\mathbb{R}^{2 \mathrm{~N}}} d \mathbf{x} d \mathbf{x}^{\prime} \Phi(\mathbf{x}) Q_{+; u}^{\mathrm{SG}}\left(\mathbf{x}, \mathbf{x}^{\prime}\right) \Psi_{t}\left(\mathbf{x}^{\prime}\right)=q_{t}^{+}(u)\left\langle\Phi \mid \Psi_{t}\right\rangle
$$

for some test-function $\Phi(\mathbf{x}) \in \mathcal{T}_{s}^{\otimes \mathrm{N}}$, where $\mathcal{T}_{s}$ is the space of test-functions which is canonically associated to the representations $\mathcal{P}_{s}$ as shown in Appendix $\mathrm{B}$ In order to find the analytic properties of $q_{t}^{+}(u)$ let us use $\mathrm{Q}_{+}^{\mathrm{SG}}(u)=\mathrm{Y}^{\mathrm{SG}}(u) \mathrm{Z}$ to represent the left hand side of $(72)$ as $\left\langle\Phi^{\prime} \mid \mathrm{Z} \Psi_{t}\right\rangle$, where

$$
\Phi^{\prime}\left(\mathbf{x}^{\prime}\right) \equiv \int_{\mathbb{R}^{\mathrm{N}}} d \mathbf{x} \Phi(\mathbf{x}) Y_{u}^{\mathrm{SG}}\left(\mathbf{x}, \mathbf{x}^{\prime}\right)
$$

With the help of the Paley-Wiener theorems one easily finds that the condition $\Phi(\mathbf{x}) \in \mathcal{T}_{s}^{\otimes N}$ implies that $\Phi(\mathbf{x})$ is entire analytic w.r.t. each variable $x_{k}$ and decays exponentially as

$$
|\Phi(\mathbf{x})| \sim e^{-\pi Q\left|x_{k}\right|} \quad \text { for }\left|x_{k}\right| \rightarrow \infty .
$$

The kernel $Y_{u}^{\mathrm{SG}}\left(\mathbf{x}, \mathbf{x}^{\prime}\right)$ has the same asymptotics w.r.t. its $x_{k}$ variables, as seen from equation 67 and relation A.25. Therefore the convergence of the integrals does not represent any problem. Combined with the observation that the left hand side of $(73)$ is the convolution of two meromorphic functions we conclude that the only source of singular behavior is the possibility that the contours of integration in (73) may become pinched between poles of the integrand approaching the contour from the upper and lower half planes, respectively. With the help of A.11 one easily compiles a list of the relevant poles of the kernel $Y_{u}^{\mathrm{SG}}\left(\mathbf{x}, \mathbf{x}^{\prime}\right)$ as given in 67):

$$
\begin{array}{cl}
\text { Upper half plane } \mathbb{H}_{-}: & \text {(1) } x_{r} \in x_{r}^{\prime}-\frac{1}{2}(u+\sigma)+\Upsilon_{0}, \\
& \text { (2) } x_{r} \in-x_{r+1}^{\prime}+\frac{1}{2}(u-\sigma)+\Upsilon_{0}, \\
\text { Lower half plane } \mathbb{H}_{+}: \quad & \left(1^{\prime}\right) x_{r} \in x_{r}^{\prime}+\frac{1}{2}(u+\sigma)-\Upsilon_{0}, \\
& \left(2^{\prime}\right) x_{r} \in-x_{r+1}^{\prime}-\frac{1}{2}(u-\sigma)-\Upsilon_{0} .
\end{array}
$$

Pinching of the contour between poles from the upper and lower half planes would produce the following series of poles:

$$
\begin{aligned}
& \left(11^{\prime}\right) \quad u+s \in+\Upsilon_{0}, \quad\left(12^{\prime}\right) \quad x_{r}^{\prime}+x_{r+1}^{\prime}-s \in-\Upsilon_{0}, \\
& \left(22^{\prime}\right) \quad u-s \in-\Upsilon_{0}, \quad\left(21^{\prime}\right) \quad x_{r}^{\prime}+x_{r+1}^{\prime}+s \in+\Upsilon_{0} .
\end{aligned}
$$

We observe in particular that none of the poles of $\Phi^{\prime}\left(\mathbf{x}^{\prime}\right)$ happens to lie on the real axis, which represents the contour of integration for each of the integrals over the variables $x_{k}^{\prime}$ in (72). Taking into 
account the exponential decay of $Y_{u}^{\mathrm{SG}}\left(\mathbf{x}, \mathbf{x}^{\prime}\right)$ for $\left|x_{k}^{\prime}\right| \rightarrow \infty$, we may conclude that the integration over $\mathrm{x}^{\prime}$ in (72) converges nicely. It follows that the left hand side of (72) defines a meromorphic function of $u$ with poles listed on the 1.h.s. of (75).

In order to verify part (ii) of the theorem let us note that $Y_{u}^{\mathrm{SG}}\left(\mathbf{x}, \mathbf{x}^{\prime}\right)$ has the asymptotic behavior

$$
Y_{u}^{\mathrm{SG}}\left(\mathbf{x}, \mathbf{x}^{\prime}\right) \sim e^{ \pm \pi i \mathrm{~N} \sigma u} \prod_{r=1}^{\mathrm{N}} e^{\mp 2 \pi i x_{r}\left(x_{r+1}^{\prime}+x_{r}^{\prime}\right)} \quad \text { for }|u| \rightarrow \infty,\left\{\begin{array}{l}
|\arg (u)|<\frac{\pi}{2} \\
|\arg (u)|>\frac{\pi}{2}
\end{array}\right.
$$

as follows straightforwardly from (A.26). In order to check that the integral obtained by exchanging the limit for $u \rightarrow \pm \infty$ with the integrations in (72) is convergent let us note that performing the integration over the variables $x_{r}$ yields the Fourier transformation $\tilde{\Phi}(\mathbf{k})$ of $\Phi(\mathbf{x})$, with argument $\mathbf{k} \equiv\left(x_{\mathbf{1}}^{\prime}+x_{2}^{\prime}, \ldots, x_{\mathrm{N}}^{\prime}+x_{\mathbf{l}}^{\prime}\right)$. Note that the change of variables $\mathbf{k}=\mathbf{k}\left(\mathbf{x}^{\prime}\right)$ is invertible for $\mathrm{N}$ odd. We may therefore represent the integration over $\mathbf{x}^{\prime}$ by an integration over $\mathbf{k}$. The nice asymptotic properties of $\tilde{\Phi}(\mathbf{k})$ which follow from our requirement $\Phi \in\left(\mathcal{T}_{s}\right)^{\otimes \mathrm{N}}$ ensure the convergence of the resulting integrals. Part (ii) of the theorem therefore follows from (76) and (66).

The proof of part (iii) of the theorem is immediate, if 64 and $\mathrm{A} .25$ are taken into account.

Remark 6. Let us comment on the nature of the problems which prevented us to determine the asymptotics of the Q-operators in the remaining cases. In both remaining cases one must observe that the change of variables $\mathbf{k}=\mathbf{k}\left(\mathbf{x}^{\prime}\right)$ is not invertible, which implies divergence of the integral over $\mathbf{x}^{\prime}$. This is closely related to the fact that the leading asymptotics of $\mathrm{T}(u)$ for $|u| \rightarrow \infty$ introduces a quasi-momentum $p_{0}$ which has purely continuous spectrum (see equations [C.13), and (C.15) in Appendix C]. It follows that $\mathrm{T}(u)$ can not have any normalizable eigenstate. Instead one should work with the spectral representation for $\mathrm{p}_{\mathrm{o}}, \mathcal{H} \simeq \int_{\mathbb{R}} d p_{\mathrm{o}} \mathcal{H}_{p_{\mathrm{o}}}$ where the elements of $\mathcal{H}_{p_{\mathfrak{o}}}$ are represented by wave-functions of the form $\Psi_{p_{\mathfrak{o}}}(\mathbf{x})=e^{2 \pi i p_{\mathfrak{o}} x_{\mathfrak{o}}} \Psi\left(x_{\mathrm{N}-1}-x_{\mathfrak{o}}, \ldots, x_{1}-x_{\mathfrak{o}}\right)$. This seems to complicate the analysis considerably. We nevertheless expect results similar to [58-ii) to hold for the remaining cases as well.

\subsection{Self-duality and quantum Wronskian relation}

The explicit form (60)- 61) of the Q-operators along with the properties of $D_{\alpha}(x)$ listed in Appendix $\mathrm{A} .3$ show that $\mathrm{Q}_{ \pm}^{b}(u)$ are self-dual with respect to the replacement $b \rightarrow b^{-1}$. Therefore, the Q-operators also satisfy the dual Baxter equations,

$$
\widetilde{\mathbf{T}}^{b}(u) \cdot \mathrm{Q}_{ \pm}^{b}(u)=\left(\tilde{a}^{b}(u)\right)^{\mathrm{N}} \mathrm{Q}_{ \pm}^{b}\left(u-i b^{-1}\right)+\left(\tilde{d}^{b}(u)\right)^{\mathrm{N}} \mathrm{Q}_{ \pm}^{b}\left(u+i b^{-1}\right),
$$

where $\widetilde{T}^{b}(u), b=\mathrm{xxz}, \mathrm{sG}$, denote the transfer-matrices corresponding to the L-matrices (10) and (33) with $b$ replaced by $b^{-1}$. These are the transfer-matrices of the modular XXZ magnet and lattice sinh-Gordon model with $\mathcal{U}_{\tilde{q}}(\mathfrak{s} l(2, \mathbb{R}))$ symmetry, where $\tilde{q}=e^{i \pi b^{-2}}$. The coefficients $\tilde{a}(u)$, $\tilde{d}(u)$ in (77) are similarly obtained from those in (62) by the replacement $b \rightarrow b^{-1}$. In the sinhGordon case the mass $m_{b^{-1}}$ is related to the representation parameter $s$ via $\frac{1}{4} m_{1 / b} \Delta=e^{-\pi b^{-1} s}$.

This self-duality has remarkable consequences, which we shall work out explicitly for the case of the sinh-Gordon model with odd N. We will take advantage of the freedom pointed out in Remark 5 to renormalize the operator $\mathrm{Q}^{\mathrm{SG}}(u)$ as follows:

$$
\check{\mathrm{Q}}(u) \equiv \mathrm{Q}_{+\infty}^{*} e^{-i \frac{\pi}{2} \mathrm{~N}\left(u^{2}+\sigma^{2}+\delta_{+}^{2}\right)} \mathbf{Q}_{+}^{S G}(u),
$$


where $\delta_{+}=\frac{1}{2}\left(b+b^{-1}\right)$ and $\mathrm{Q}_{+\infty}$ is the unitary operator which appears in the asymptotics (70). The Baxter equation for $\mathrm{Q}(u)$ will then take the following form:

$$
\begin{gathered}
\mathrm{T}^{S G}(u) \cdot \check{\mathrm{Q}}(u)=(\check{a}(u))^{\mathrm{N}} \check{\mathrm{Q}}(u-i b)+(\check{d}(u))^{\mathrm{N}} \check{\mathrm{Q}}(u+i b), \\
\text { where } \check{d}(u)=\check{a}(-u)=1+\left(\frac{m \Delta}{4}\right)^{2} e^{-\pi b(2 u+i b)} .
\end{gathered}
$$

The normalization of the operator $\check{\mathrm{Q}}(u)$ was for later convenience chosen in such a way that $\check{\mathrm{Q}}(u) \sim e^{ \pm \pi i \sigma u-i \frac{\pi}{2} \mathrm{~N}\left(u^{2}+\sigma^{2}+\delta_{+}^{2}\right)} \cdot 1$ for $\operatorname{Re}(u) \rightarrow \pm \infty$.

Theorem 4. The operator $\mathrm{Q}(u)$ fulfills the following quantum Wronskian relation:

$$
\check{\mathrm{Q}}\left(u+i \delta_{+}\right) \check{\mathrm{Q}}\left(u-i \delta_{+}\right)-\check{\mathrm{Q}}\left(u+i \delta_{-}\right) \check{\mathrm{Q}}\left(u-i \delta_{-}\right)=W_{\mathrm{N}}(u) \cdot 1 .
$$

where $W_{\mathrm{N}}(u)=e^{-i \pi \mathrm{N}\left(u^{2}+\sigma^{2}\right)}\left(D_{\sigma}(u)\right)^{-\mathrm{N}}$ and $\delta_{ \pm} \equiv \frac{1}{2}\left(b^{-1} \pm b\right)$.

Proof. Let W $(u)$ be the left hand side of 80 . A straightforward calculation, using the Baxter equation and its dual form, shows that $\mathrm{W}(u)$ satisfies the following two functional relations:

$$
\mathrm{W}\left(u+\frac{i}{2} b^{ \pm 1}\right)=\left(e^{2 \pi b^{ \pm 1} u} \frac{\cosh \pi b^{ \pm 1}(u-\sigma)}{\cosh \pi b^{ \pm 1}(u+\sigma)}\right)^{\mathrm{N}} \mathrm{W}\left(u-\frac{i}{2} b^{ \pm 1}\right)
$$

A solution to both functional relations is given by the expression on the right hand side of 80 . If $b$ is irrational it suffices to notice that $\mathrm{W}(u)$ is meromorphic in order to conclude that the solution to the system 81 must be unique up to multiplication by an operator which does not depend on $u$. This freedom can be fixed by comparing the asymptotics of both sides for $\operatorname{Re}(u) \rightarrow \pm \infty$ using equations (70), (71) (for considering the asymptotics as $\operatorname{Re}(u) \rightarrow-\infty$, it is helpful to notice that $\Omega^{2}=1$ ). In order to cover the case of rational $b$ let us notice that both sides of the relation 80 , can be analytically continued from irrational values of $b$ to the case where $b$ is rational.

From the proof of this theorem it is clear that the main ingredient is the self-duality of our representations $\mathcal{P}_{s}$. We therefore expect that a similar result will hold for the remaining cases as well. However, at present we do not control the asymptotics of the Q-operators sufficiently well in these cases.

\subsection{Parity and cyclic shift}

Let us consider the cyclic shift and parity operators $U$ and $\Omega$ defined respectively by

$$
\mathrm{U} f\left(x_{1}, x_{2}, \ldots, x_{\mathrm{N}}\right)=f\left(x_{2}, \ldots, x_{\mathrm{N}}, x_{1}\right), \quad \Omega f\left(x_{1}, \ldots, x_{\mathrm{N}}\right)=f\left(-x_{1}, \ldots,-x_{\mathrm{N}}\right) .
$$

These operators commute with each other and also with $Q_{ \pm}^{b}(u)$ (as can be easily seen from (60)(61). Hence they must commute with $\mathrm{T}_{ \pm}^{b}(u)$, as can also be verified directly ${ }^{2}$. It follows that eigenstates $\Psi_{t}$ may be assumed to be simultaneously eigenstates of $U$ and $\Omega$,

$$
\Omega \Psi_{t}= \pm \Psi_{t}, \quad \mathrm{U} \Psi_{t}=e^{2 \pi i \frac{m}{\mathrm{~N}}} \Psi_{t}, \quad m=1, \ldots, \mathrm{N} .
$$

\footnotetext{
${ }^{2}$ Indeed, for $\mathrm{U}$ this is obvious from the definition 17 and for $\Omega$ it follows from the observation that $\Omega L^{\mathrm{xxz}}(u) \Omega=$ $\sigma_{1} e^{-\pi b u \sigma_{3}} L^{\mathrm{Xxz}}(u) e^{\pi b u \sigma_{3}} \sigma_{1}$ and $\Omega L^{\mathrm{SG}}(u) \Omega=\sigma_{1} L^{\mathrm{SG}}(u) \sigma_{1}$.
} 
It is therefore useful to observe that $U$ and $\Omega$ can be recovered from $Q_{ \pm}^{b}(u)$ as follows. First one may notice that the integral kernel (60) simplifies for the special values $u= \pm \sigma$ thanks to the relation A.30. Explicitely, we have

$$
\mathrm{Q}_{+}^{b}(\sigma)=\left(w_{b}\left(2 s+\frac{i}{2} Q\right)\right)^{-\mathrm{N}} \cdot 1, \quad \mathrm{Q}_{+}^{b}(-\sigma)=\left(w_{b}\left(2 s+\frac{i}{2} Q\right)\right)^{-\mathrm{N}} \cdot \mathrm{U}-1 \cdot \Omega^{b},
$$

where $\Omega^{b}$ is defined in $($ E.32). Combining this observation with 83 , we conclude that

$$
q_{t}^{+}(\sigma)=\left(w_{b}\left(2 s+\frac{i}{2} Q\right)\right)^{-\mathrm{N}}, \quad q_{t}^{+}(-\sigma)= \pm e^{2 \pi i \frac{m}{\mathrm{~N}}}\left(w_{b}\left(2 s+\frac{i}{2} Q\right)\right)^{-\mathrm{N}}
$$

where the - sign in the second expression can occur only in the sinh-Gordon model.

Secondly let us observe that eqs. [65] and (85) imply that $u= \pm \bar{\sigma}$ are poles of order $\mathrm{N}$ for $q_{t}^{-}(u)$. Indeed, using 61, (A.30, and , we find

$$
\mathrm{Q}_{-}^{\mathrm{b}}(\bar{\sigma}+\epsilon) \sim\left(\frac{i}{2 \pi \epsilon}\right)^{\mathrm{N}} \cdot 1, \quad \mathrm{Q}_{-}^{\mathrm{b}}(-\bar{\sigma}+\epsilon) \sim\left(\frac{1}{2 \pi i \epsilon}\right)^{\mathrm{N}} \cdot \Omega^{\mathrm{b}} \cdot \mathrm{U}
$$

as $\epsilon \rightarrow 0$. This implies

$$
q_{t}^{-}(\bar{\sigma}+\epsilon) \sim\left(\frac{i}{2 \pi \epsilon}\right)^{\mathrm{N}}, \quad q_{t}^{-}(-\bar{\sigma}+\epsilon) \sim \pm e^{2 \pi i \frac{m}{\mathrm{~N}}}\left(\frac{1}{2 \pi i \epsilon}\right)^{\mathrm{N}}
$$

where the - sign in the second expression can occur again only in the sinh-Gordon model.

\section{Separation of variables}

In the previous section we have identified necessary conditions for a function $t(u)$ to be an eigenvalue of $\mathrm{T}(u)$. If we were able to show that these conditions are also sufficient, we would have arrived at a useful reformulation of the Auxiliary Spectral Problem.

A promising approach to this problem is offered by the separation of variables method pioneered by Sklyanin [S2] [S3]. The basic idea is to introduce a representation for the Hilbert space $\mathcal{H}$ of the model in which the off-diagonal element of the monodromy matrix $\mathrm{M}(u)$, the operator $\mathrm{B}(u)$, is diagonal.

For simplicity of exposition let us temporarily restrict attention to the case of the sinh-Gordon model with $\mathrm{N}$ odd. The operator $\mathrm{B}(u)$ has the following form:

$$
\mathrm{B}(u)=-i^{\mathrm{N}} e^{\mathrm{N} \pi b(u-s)} \sum_{m=0}^{\mathrm{N}}(-)^{m} e^{-2 m \pi b u} \mathrm{~B}_{m} .
$$

By Lemma [5] the operators $\mathrm{B}_{m}, m=0, \ldots, \mathrm{N}$ are positive self-adjoint. Basic for the separation of variables method is the validity of the following conjecture.

Conjecture 1. The joint spectrum of the family of operators $\left\{\mathrm{B}_{m} ; m=0, \ldots, \mathrm{N}\right\}$ is simple. This means that eigenstates of $\mathrm{B}(u)$ are uniquely parameterized by the corresponding eigenvalue $b(u)$.

This conjecture can be supported by counting the degrees of freedom. However, it is not easy to provide a rigorous proof (see also Remark 7 below). 
The function $e^{-\pi b \mathrm{~N} u} b(u)$ is a polynomial in the variable $\lambda=e^{-2 \pi b u}$. It can conveniently be represented in the following form

$$
b(u)=-(2 i)^{\mathrm{N}} e^{-\pi b \mathrm{~N} s} \prod_{k=1}^{\mathrm{N}} \sinh \pi b\left(u-y_{k}\right) .
$$

The variables $y_{k}, k=1, \ldots, \mathrm{N}$ are uniquely defined up to permutations once we adopt the convention that $\operatorname{Im}\left(y_{k}\right) \in\left(-\frac{1}{2 b}, \frac{1}{2 b}\right]$. This means that the representation for the Hilbert space $\mathcal{H}$ in which $\mathrm{B}(u)$ is diagonal may be described by wave-functions $\Psi(\mathbf{y}), \mathbf{y}=\left(y_{1}, \ldots, y_{\mathrm{N}}\right)$. This representation for the vectors in $\mathcal{H}$ will subsequently be referred to as the SOV representation.

We will then show that the Auxiliary Spectral Problem, T $(u) \Psi_{t}=t(u) \Psi_{t}$, gets transformed into the system of Baxter equations

$$
t\left(y_{k}\right) \Psi(\mathbf{y})=\left[\left(a\left(y_{k}\right)\right)^{\mathrm{N}} \mathrm{T}_{k}^{-}+\left(d\left(y_{k}\right)\right)^{\mathrm{N}} \mathrm{T}_{k}^{+}\right] \Psi(\mathbf{y}), \quad k=1, \ldots, \mathrm{N},
$$

where the operators $T_{k}$ are shift operators defined as

$$
\mathrm{T}_{k}^{ \pm} \Psi(\mathbf{y}) \equiv \Psi\left(y_{1}, \ldots, y_{k} \pm i b, \ldots, y_{\mathrm{N}}\right) .
$$

The coefficients in front of the shift operators in (90) depend only on a single variable $y_{k}$, which is the crucial simplification that is gained by working in the representation where $\mathrm{B}(u)$ is diagonal.

The key observation to be made at this point is that the same finite difference equation (90) was found in the previous Section 4 in connection with the necessary conditions for a function $t(u)$ to represent a point in the spectrum. It now remains to observe that any function $q_{t}(u)$ that fulfills the necessary conditions (57), (58) can be used to construct

$$
\Psi_{t}(\mathbf{y})=\prod_{k=1}^{\mathrm{N}} q_{t}\left(y_{k}\right)
$$

The fact that (92) defines an eigenstate of $\mathrm{T}(u)$ is verified by comparing (90) with (57). The main point that needs to be verified is whether the function $\Psi_{t}(\mathbf{y})$ actually represents an element of $\mathcal{H}$, i.e. whether it has finite norm. The scalar product of vectors in $\mathcal{H}$ can be represented in the form

$$
\left\langle\Psi_{2} \mid \Psi_{1}\right\rangle=\int_{\mathbb{Y}} d \mu(\mathbf{y})\left\langle\Psi_{2} \mid \mathbf{y}\right\rangle\left\langle\mathbf{y} \mid \Psi_{1}\right\rangle
$$

We clearly need to know both the range $\mathbb{Y}$ of values $\mathbf{y}$ that we need to integrate over, as well as the measure $d \mu(\mathbf{y})$ of integration to be used. We will be able to determine the measure $d \mu(\mathbf{y})$ provided that the following conjecture is true.

Conjecture 2. The functions $b(u)$ of the product form (89) that describe the spectrum of $\mathrm{B}(u)$ have only real roots, i.e., $y_{k} \in \mathbb{R}$ for $k=1, \ldots, N$.

We will discuss the status of this conjecture after having explained its consequences. Conjecture 2 directly implies that $\mathbb{Y}=\mathbb{R}^{\mathrm{N}}$ in 93 . Assuming the validity of Conjecture 2 we will show in Proposition 2 that the measure $d \mu(\mathbf{y})$ can be represented in the following explicit form

$$
d \mu(\mathbf{y})=\prod_{k=1}^{\mathrm{N}} d y_{k} \prod_{l<k} 4 \sinh \pi b\left(y_{k}-y_{l}\right) \sinh \pi b^{-1}\left(y_{k}-y_{l}\right) .
$$


Knowing explicitly how to represent the scalar product of $\mathcal{H}$ in the SOV representation finally allows us to check that any solution of the necessary conditions (57), (58) defines an eigenvector $\left|\Psi_{t}\right\rangle$ of $\mathrm{T}(u)$ via (92). In other words: The conditions (57), (58) are not only necessary but also sufficient for $t(u)$ to be an eigenvalue of a vector $\left|\Psi_{t}\right\rangle \in \mathcal{H}$.

Remark 7. Our claim that the conditions (57), 58] are also sufficient for a function $t(u)$ to represent a point in the spectrum of $\mathrm{T}(u)$ does not seem to depend very strongly on the validity of the Conjecture 2 In this sense the conjecture mainly serves us to simplify the exposition.

In any case it is a problem of fundamental importance for the separation of variable method to determine the spectrum of $\mathrm{B}(u)$ precisely. Even in simpler models which have been studied along similar lines like the Toda chain [ $[\mathrm{KL}]$ or the XXX spin chains [DKM] there does not seem to exist a rigorous proof of the analogous statements. The explicit construction of the eigenfunctions of $\mathrm{B}(u)$, which may proceed along similar lines as followed for the Toda chain in $[\mathrm{KL}]$ or for the XXX chain in [DKM], should provide us with the basis for a future proof of Conjecture 2] or some modification thereof.

Remark 8. Within the Separation of Variables method the Auxiliary Spectral Problem gets transformed into the separated Baxter equations 90 . However, these finite difference equations will generically have many solutions that do not correspond to eigenstates of $\mathrm{T}(u)$.

In order to draw a useful analogy let us compare the situation with the spectral problem for a differential operator like $\mathrm{h}=-\partial_{y}^{2}+V(y)$. One generically has two linearly independent solutions to the second order differential equation like $\left(-\partial_{y}^{2}+V(y)\right) \psi=\mathcal{E} \psi$ for any choice of $\mathcal{E}$. The spectrum of $h$ is determined by restricting attention to the subset of square-integrable solutions within the set of all solutions to the eigenvalue equation.

From this point of view we may identify the conditions (58) on analyticity and asymptotics of the function $q_{t}(u)$ as the quantization conditions which single out the subset which constitutes the spectrum of $\mathrm{T}(u)$ among the set of all solutions of 90).

\subsection{Operator zeros of $\mathrm{B}(u)$}

The adaption of Sklyanin's observation to the case at hand is based on the following observations. First, by Lemma 5 the operators $B_{m}$ introduced in (88) are positive self-adjoint. Taking into account the mutual commutativity (which follows from (100),

$$
\left[\mathrm{B}_{m}, \mathrm{~B}_{n}\right]=0
$$

leads us to conclude that the family of operators $\left\{\mathrm{B}_{m} ; m=1, \ldots \mathrm{N}\right\}$ can be simultaneously diagonalized $^{3}$.

Conjecture 1 implies that the spectral representation for the family $\left\{\mathrm{B}_{m} ; m=1, \ldots \mathrm{N}\right\}$ can be written in the form

$$
|\Psi\rangle=\int_{\mathbb{R}_{+}^{N}} d \nu(\mathbf{b})|\mathbf{b}\rangle\langle\mathbf{b} \mid \Psi\rangle
$$

where $|\mathbf{b}\rangle$ is a (generalized) eigenvector of $\mathrm{B}_{m}$ with eigenvalue $b_{m}$, and we have assembled the eigenvalues into the vector $\mathbf{b}=\left(b_{1}, \ldots, b_{\mathrm{N}}\right)$.

\footnotetext{
${ }^{3}$ By Lemma 6 we have $B_{0}=\left(B_{N}\right)^{-1}$.
} 
It now turns out to be particularly useful to parameterize the polynomial of eigenvalues $b(u)$ in terms of its roots. This representation may always be written as follows

$$
b(u) \equiv b(u \mid \mathbf{y}) \equiv-(2 i)^{\mathrm{N}} e^{-\pi b \mathrm{~N} s} \prod_{k=1}^{\mathrm{N}} \sinh \pi b\left(u-y_{k}\right),
$$

where $\mathbf{y}=\left(y_{1}, \ldots, y_{\mathrm{N}}\right)$. The variables $y_{k}$ are either real or they come in pairs related by complex conjugation. The variables $y_{k}$ are uniquely defined up to permutations if one requires that $\operatorname{Im}\left(y_{k}\right) \in\left(-\frac{1}{2 b}, \frac{1}{2 b}\right]$. We will assume that $y_{k} \in \mathbb{R}$ according to Conjecture 2 It then follows that the spectral representation 96 can be rewritten as

$$
|\Psi\rangle=\int_{\mathbb{R}^{\mathrm{N}}} d \mu(\mathbf{y})|\mathbf{y}\rangle\langle\mathbf{y} \mid \Psi\rangle
$$

However, points $\mathbf{y}, \mathbf{y}^{\prime}$ in $\mathbb{R}^{N}$ which are obtained from each other by the permutation $y_{k} \leftrightarrow y_{l}$ will correspond to the same eigenstate of $\mathrm{B}(u)$. This means that the spectral representation for $\mathrm{B}(u)$ can be used to define an isomorphism

$$
\mathcal{H} \simeq L^{2}\left(\mathbb{R}^{\mathrm{N}} ; d \mu\right)^{\mathrm{Symm}}
$$

where $L^{2}\left(\mathbb{R}^{\mathrm{N}} ; d \mu\right)^{\text {Symm }}$ is the subspace within $L^{2}\left(\mathbb{R}^{\mathrm{N}} ; d \mu\right)$ which consists of totally symmetric wave-functions.

Despite the fact that $\mathcal{H}$ is isomorphic only to a subspace in $L^{2}\left(\mathbb{R}^{\mathrm{N}} ; d \mu\right)$ it will turn out to be useful to extend the definition of the operators $\mathrm{A}(u), \mathrm{B}(u), \mathrm{C}(u), \mathrm{D}(u)$ from $L^{2}\left(\mathbb{R}^{\mathrm{N}} ; d \mu\right)^{\mathrm{Symm}}$, where it is canonically defined via (99) to $L^{2}\left(\mathbb{R}^{\mathrm{N}} ; d \mu\right)$. As a first step let us introduce the operators $\mathrm{y}_{k}$ which act as $\mathrm{y}_{k}|\mathbf{y}\rangle=y_{k}|\mathbf{y}\rangle$. Substituting $y_{k} \rightarrow \mathrm{y}_{k}$ in (97) leads to a representation of the operator $\mathrm{B}(u)$ in terms of its operators zeros $\mathrm{y}_{k}$.

\subsection{Operators $\mathrm{A}(u)$ and $\mathrm{D}(u)$}

Monodromy matrices of the modular XXZ magnet and the lattice sinh-Gordon model satisfy the exchange relations (12), where the R-matrix is given by (15) and (34), respectively. Among these relations we have, in particular, the following

$$
\begin{aligned}
& {[\mathrm{B}(u), \mathrm{B}(v)]=0, \quad[\mathrm{~A}(u), \mathrm{A}(v)]=0, \quad[\mathrm{D}(u), \mathrm{D}(v)]=0,} \\
& \sinh \pi b(u-v+i b) \mathrm{B}(u) \mathrm{A}(v) \\
& =\sinh \pi b(u-v) \mathrm{A}(v) \mathrm{B}(u)+R_{23}^{b}(u-v) \mathrm{B}(v) \mathrm{A}(u), \\
& \sinh \pi b(u-v+i b) \mathrm{B}(v) \mathrm{D}(u) \\
& =\sinh \pi b(u-v) \mathrm{D}(u) \mathrm{B}(v)+R_{32}^{b}(u-v) \mathrm{B}(u) \mathrm{D}(v), \\
& R_{23}^{\mathrm{Xxz}}(u)=R_{32}^{\mathrm{xxz}}(-u)=i e^{\pi b u} \sin \gamma, \quad R_{23}^{\mathrm{SG}}(u)=R_{32}^{\mathrm{SG}}(u)=i \sin \gamma .
\end{aligned}
$$

We are now going to show that there is an essentially unique representation of the commutation relations (100)- (102) on wave-functions $\Psi(\mathbf{y})$ which is such that $\mathrm{B}(u)$ is represented as operator of multiplication by $b(u) \equiv b(u \mid \mathbf{y})$, cf. (97). 
To this aim let us consider for $k \geq 1$ the following distributions:

$$
\langle\mathbf{y}| \mathrm{A}\left(y_{k}\right) \equiv \lim _{u \rightarrow y_{k}}\langle\mathbf{y}| \mathrm{A}(u), \quad\langle\mathbf{y}| \mathrm{D}\left(y_{k}\right) \equiv \lim _{u \rightarrow y_{k}}\langle\mathbf{y}| \mathrm{D}(u)
$$

These distributions, which will be defined on suitable dense subspaces of $L^{2}\left(\mathbb{R}^{\mathrm{N}}, d \mu\right)$, can be regarded as the result of action on $\langle\mathbf{y}|$ by operators $\mathrm{A}\left(\mathrm{y}_{k}\right)$ and $\mathrm{D}\left(\mathrm{y}_{k}\right)$ with operator arguments substituted into (C.3), (C.5), (C.6), (C.8) from the left. The commutation relations (101)-(102) imply that $\langle\mathbf{y}| \mathrm{A}\left(y_{k}\right)$ and $\langle\mathbf{y}| \mathrm{D}\left(y_{k}\right)$ are eigenstates of $\mathrm{B}(u)$ with eigenvalues $b\left(u \mid \mathbf{y}^{\prime}\right)$, with $y_{k}^{\prime}=y_{k} \mp i b$, respectively, $y_{l}^{\prime}=y_{l}$ otherwise. This, along with relations 100, leads to the conclusion that the action of the operators $\mathrm{A}\left(\mathrm{y}_{k}\right)$ and $\mathrm{D}\left(\mathrm{y}_{k}\right)$ on wave-functions $\Psi(\mathbf{y})=\langle\mathbf{y} \mid \Psi\rangle$ can be represented in the form

$$
\mathrm{A}\left(\mathrm{y}_{k}\right) \Psi(\mathbf{y})=a_{\mathrm{N}, k}\left(y_{k}\right) \mathrm{T}_{k}^{-} \Psi(\mathbf{y}), \quad \mathrm{D}\left(\mathrm{y}_{k}\right) \Psi(\mathbf{y})=d_{\mathrm{N}, k}\left(y_{k}\right) \mathrm{T}_{k}^{+} \Psi(\mathbf{y}),
$$

where $\mathrm{T}_{k}^{ \pm}$are the shift operators defined in equation (91). The functions $a_{\mathrm{N}, k}\left(y_{k}\right)$ and $d_{\mathrm{N}, k}\left(y_{k}\right)$ are further restricted by the following identities:

$$
\begin{aligned}
\operatorname{det}_{q} \mathrm{M}^{\mathrm{SG}}(u) & \equiv \mathrm{A}^{\mathrm{SG}}(u) \mathrm{D}^{\mathrm{SG}}(u-i b)-\mathrm{B}^{\mathrm{SG}}(u) \mathrm{C}^{\mathrm{SG}}(u-i b) \\
& =\left(4 e^{-2 \pi b s} \cosh \pi b\left(s+u-i \frac{b}{2}\right) \cosh \pi b\left(s-u+i \frac{b}{2}\right)\right)^{\mathrm{N}} .
\end{aligned}
$$

These identities are proven in Appendix C.2 It follows then from 104 that $\operatorname{det}_{q} \mathrm{M}\left(y_{k}\right)=$ $a_{\mathrm{N}, k}\left(y_{k}\right) d_{\mathrm{N}, k}\left(y_{k}-i b\right)$. Not having specified the measure $\mu(\mathbf{y})$ yet leaves us the freedom to multiply all wave-functions $\Psi(\mathbf{y})$ by functions of the form $\prod_{k} f_{k}\left(y_{k}\right)$. This allows us to choose

$$
\begin{aligned}
& \begin{aligned}
a_{\mathrm{N}, k}\left(y_{k}\right)=\left(a\left(y_{k}\right)\right)^{\mathrm{N}}, & d_{\mathrm{N}, k}\left(y_{k}\right)=\left(d\left(y_{k}\right)\right)^{\mathrm{N}}
\end{aligned} \\
& \text { where } a^{\mathrm{SG}}(u)=d^{\mathrm{SG}}(-u)=e^{-\pi b s} 2 \cosh \pi b\left(u-s-i \frac{b}{2}\right) \\
& =e^{-\pi b\left(u-i \frac{b}{2}\right)}+\left(\frac{m \Delta}{4}\right)^{2} e^{\pi b\left(u-i \frac{b}{2}\right)} .
\end{aligned}
$$

We have used that the sinh-Gordon parameters $m, \Delta$ are related to $s$ as in (49).

In the special case that $\Psi(\mathbf{y})$ is an eigenfunction of the transfer-matrix $\mathrm{T}(u)$ with eigenvalue $t(u)$ we get the Baxter equations (90) from $\mathrm{T}\left(\mathrm{y}_{k}\right)=\mathrm{A}\left(\mathrm{y}_{k}\right)+\mathrm{D}\left(\mathrm{y}_{k}\right)$ and equations (104) and (107), as advertised.

It will be useful for us to have explicit formulae for $\mathrm{A}(u)$ and $\mathrm{D}(u)$ in terms of the operators $\mathrm{y}_{k}$ and $\mathrm{T}_{k}^{ \pm}$. In the case of $\mathrm{N}$ odd we may use the following formulae:

$$
\begin{aligned}
& \mathrm{A}^{\mathrm{SG}}(u)=\sum_{k=1}^{\mathrm{N}} \prod_{l \neq k} \frac{\sinh \pi b\left(u-\mathrm{y}_{l}\right)}{\sinh \pi b\left(\mathrm{y}_{k}-\mathrm{y}_{l}\right)}\left(a^{\mathrm{SG}}\left(\mathrm{y}_{k}\right)\right)^{\mathrm{N}} \mathrm{T}_{k}^{-}, \\
& \mathrm{D}^{\mathrm{SG}}(u)=\sum_{k=1}^{\mathrm{N}} \prod_{l \neq k} \frac{\sinh \pi b\left(u-\mathrm{y}_{l}\right)}{\sinh \pi b\left(\mathrm{y}_{k}-\mathrm{y}_{l}\right)}\left(d^{\mathrm{SG}}\left(\mathrm{y}_{k}\right)\right)^{\mathrm{N}} \mathrm{T}_{k}^{+} .
\end{aligned}
$$

These formulae are easily verified by noting that the number of variables $\mathrm{y}_{k}$ coincides with the number of coefficients in the expansion (C.6) and (C.8). It follows that the polynomials $A^{\mathrm{SG}}(u)$ and $\mathrm{D}^{\mathrm{SG}}(u)$ are uniquely determined by their values $\mathrm{A}^{\mathrm{SG}}\left(\mathrm{y}_{k}\right)$ and $\mathrm{D}^{\mathrm{SG}}\left(\mathrm{y}_{k}\right), k=1, \ldots, \mathrm{N}$. 


\subsection{Sklyanin measure}

We furthermore know that the operators $A_{m}$ and $D_{m}$ which are defined by the expansion

$$
\begin{aligned}
& \mathrm{A}^{\mathrm{SG}}(u)=-i e^{-\pi b u} i^{\mathrm{N}} e^{\pi b \mathrm{~N}(u-s)} \sum_{m=0}^{\mathrm{N}-1}(-)^{m} e^{-2 m \pi b u} \mathrm{~A}_{m}, \\
& \mathrm{D}^{\mathrm{SG}}(u)=-i e^{-\pi b u} i^{\mathrm{N}} e^{\pi b \mathrm{~N}(u-s)} \sum_{m=0}^{\mathrm{N}-1}(-)^{m} e^{-2 m \pi b u} \mathrm{D}_{m}
\end{aligned}
$$

are positive (Lemma 5 in Appendix C).

Proposition 2. There exists a unique measure $d \mu(\mathbf{y})$ such that the operators $\mathrm{A}_{m}$ and $\mathrm{D}_{m}$ on $L^{2}\left(\mathbb{R}^{\mathrm{N}} ; d \mu\right)$ are positive. This measure $d \mu$ can be represented explicitly as

$$
d \mu(\mathbf{y})=\prod_{k=1}^{\mathrm{N}} d y_{k} \prod_{l<k} 4 \sinh \pi b\left(y_{k}-y_{l}\right) \sinh \pi b^{-1}\left(y_{k}-y_{l}\right)
$$

Proof. The similarity transformation $\Psi(\mathbf{y})=\chi_{\mathrm{A}}(\mathbf{y}) \Phi(\mathbf{y})$, where

$$
\chi_{\mathbf{A}}(\mathbf{y})=\prod_{k=1}^{\mathrm{N}}\left(e^{\pi i y_{k} s} w_{b}\left(y_{k}-s\right)\right)^{-\mathrm{N}} \prod_{l<k}\left(w_{b}\left(y_{k}-y_{l}+\frac{i}{2} Q\right)\right)^{-1},
$$

maps to a representation in which the operator $\mathrm{A}^{\mathrm{SG}}(u)$ is represented as

$$
\mathrm{A}^{\mathrm{SG}}(u)=\sum_{k=1}^{\mathrm{N}} \prod_{l \neq k} \sinh \pi b\left(u-\mathrm{y}_{l}\right) \mathrm{T}_{k}^{-} .
$$

Expanding in powers of $e^{\pi b u}$ yields a representation for the coefficients $\mathrm{A}_{m}^{\mathrm{SG}}$ that appear in the expansion (C.3 which takes the form

$$
\mathrm{A}_{m}^{\mathrm{SG}}=\sum_{k=1}^{\mathrm{N}} p_{m k}(\mathbf{y}) \mathrm{T}_{k}^{-},
$$

The coefficients $p_{m k}(\mathbf{y})$ in (116) are positive for all $\mathbf{y} \in \mathbb{R}^{\mathrm{N}}$, and $p_{m k}(\mathbf{y})$ does not depend on $y_{k}$. We are next going to show that the positivity of $A_{m}^{S G}$ implies that $\mathrm{T}_{k}^{-}$must be a positive operator in $L^{2}\left(\mathbb{R}^{\mathrm{N}} ; d \mu\right)$. Let us keep in mind that $\mathrm{T}_{k}^{-}$satisfies the commutation relations

$$
e^{-i t \mathrm{y}_{l}} \mathrm{~T}_{k}^{-} e^{i t \mathrm{y}_{l}}=e^{b t \delta_{k l}} \mathrm{~T}_{k}^{-} .
$$

If there was any negative contribution to the expectation value $\left\langle\Phi\left|\sum_{k=1}^{\mathrm{N}} p_{m k}(\mathbf{y}) \mathrm{T}_{k}^{-}\right| \Phi\right\rangle$ we could make it arbitrarily large by means of the unitary transformation $|\Phi\rangle \rightarrow e^{i t y_{k}}|\Phi\rangle$. It follows that $\left\langle\Phi\left|p_{m k}(\mathbf{y}) \mathrm{T}_{k}^{-}\right| \Phi\right\rangle>0$ for any $k=1, \ldots, \mathrm{N}$.

It remains to notice that, since the $p_{m k}(\mathbf{y})$ are non-vanishing, vectors of the form $\sqrt{p_{m k}(\mathbf{y})}|\Phi\rangle$ form a dense subset in $L^{2}\left(\mathbb{R}^{\mathrm{N}} ; d \mu\right)$. This finally allows us to conclude that $\mathrm{T}_{k}^{-}$must be a positive operator. But this furthermore implies that $\left(\mathrm{T}_{k}^{-}\right)^{i l}$ is a unitary operator which satisfies the commutation relations

$$
\left(\mathrm{T}_{k}^{-}\right)^{i l} e^{i t y_{m}}=\exp \left(i b l t \delta_{k m}\right) e^{i t y_{m}}\left(\mathbf{T}_{k}^{-}\right)^{i l}
$$


It is well-known that the representation of these commutation relations by unitary operators is essentially unique. The measure which defines the corresponding Hilbert space is just $d \nu(\mathbf{y})=$ $\prod_{k=1}^{\mathrm{N}} d y_{k}$.

It remains to return in (98) to the original representation via (114), that is to compute $d \mu(\mathbf{y})=\left|\chi_{\mathbf{A}}(\mathbf{y})\right|^{2} d \nu(\mathbf{y})$. Using A.7 A.10 to simplify the resulting expression yields the formula for $d \mu(\mathbf{y})$ stated in Proposition 2

For the operator $D^{\mathrm{SG}}(u)$, a completely analogous consideration applies with the transformation $\Psi(\mathbf{y})=\chi_{\mathrm{D}}(\mathbf{y}) \Phi(\mathbf{y})$, where

$$
\chi_{\mathrm{D}}(\mathbf{y})=\prod_{k=1}^{\mathrm{N}}\left(e^{-\pi i y_{k} s} w_{b}\left(y_{k}+s\right)\right)^{\mathrm{N}} \prod_{l<k}\left(w_{b}\left(y_{k}-y_{l}+\frac{i}{2} Q\right)\right)^{-1} .
$$

Thanks to the relation A.10) and the Conjecture 2 we have $\left|\chi_{\mathrm{A}}(\mathbf{y})\right|^{2}=\left|\chi_{\mathrm{D}}(\mathbf{y})\right|^{2}$. This leads to the same measure $d \mu(\mathbf{y})$ given by (113).

\subsection{Remaining cases}

To end this section let us briefly discuss the necessary modification in the cases of the modular XXZ magnet and the lattice sinh-Gordon model with even $\mathrm{N}$. The main new feature that arises in these cases is the existence of a quasi-momentum $y_{0}$ which first appears in the expansions

$$
\begin{aligned}
& b^{\mathrm{xxz}}(u)=2^{\mathrm{N}-1} i e^{\pi b\left(u+y_{\mathrm{o}}\right)} \prod_{k=1}^{\mathrm{N}-1} \sinh \pi b\left(u-y_{k}\right), \\
& b^{\mathrm{SG}}(u)=-(2 i)^{\mathrm{N}-1} e^{\pi b\left(y_{\mathrm{o}}-\mathrm{N} s\right)} \prod_{k=1}^{\mathrm{N}-1} \sinh \pi b\left(u-y_{k}\right), \quad \mathrm{N}-\text { even },
\end{aligned}
$$

The variable $y_{\mathrm{o}}$ requires a slightly different treatment compared to the $y_{k}, k \geq 1$. Considering the states (where $\kappa=0$ for the modular magnet and $\kappa=i / 2+b s$ for the sinh-Gordon model with even $\mathrm{N}$ )

$$
\begin{aligned}
& \langle\mathbf{y}| \mathrm{A}_{\mathcal{o}} \equiv \lim _{u \rightarrow+\infty} e^{\pi \mathrm{N}(\kappa-b u)}\langle\mathbf{y}| \mathrm{A}(u), \\
& \langle\mathbf{y}| \mathrm{D}_{\mathcal{o}} \equiv \lim _{u \rightarrow+\infty} e^{\pi \mathrm{N}(\kappa-b u)}\langle\mathbf{y}| \mathrm{D}(u), \\
& \langle\mathbf{y}| \mathrm{A}_{\mathrm{N}} \equiv \lim _{u \rightarrow-\infty}(-)^{\mathrm{N}} e^{\pi \mathrm{N}(\kappa+b u)}\langle\mathbf{y}| \mathrm{A}(u), \\
& \langle\mathbf{y}| \mathrm{D}_{\mathrm{N}} \equiv \lim _{u \rightarrow-\infty}(-)^{\mathrm{N}} e^{\pi \mathrm{N}(\kappa+b u)}\langle\mathbf{y}| \mathrm{D}(u),
\end{aligned}
$$

and taking into account the asymptotic behaviour of $\mathrm{B}(u)$ and of the coefficients 103 at $u \rightarrow \pm \infty$, we infer from the relations 101 102 that

$$
\begin{array}{cc}
\mathrm{A}_{\mathfrak{o}} \Psi(\mathbf{y})=a_{\mathfrak{o}}\left(y_{\mathfrak{o}}\right) \mathrm{T}_{\mathfrak{o}}^{+} \Psi(\mathbf{y}), & \mathrm{D}_{\mathcal{o}} \Psi(\mathbf{y})=d_{\mathfrak{o}}\left(y_{\mathfrak{o}}\right) \mathrm{T}_{\mathfrak{o}}^{-} \Psi(\mathbf{y}), \\
\mathrm{A}_{\mathrm{N}} \Psi(\mathbf{y})=a_{\mathrm{N}}\left(y_{\mathfrak{o}}\right) \mathrm{T}_{\mathcal{o}}^{-} \Psi(\mathbf{y}), & \mathrm{D}_{\mathrm{N}} \Psi(\mathbf{y})=d_{\mathrm{N}}\left(y_{\mathfrak{o}}\right) \mathrm{T}_{\mathfrak{o}}^{+} \Psi(\mathbf{y}),
\end{array}
$$

where the shift operators $\mathrm{T}_{\mathrm{o}}^{ \pm}$are defined analogously to 91 for the variable $y_{\mathrm{o}}$. It follows from (C.13) and (C.15) that we have $a_{\mathfrak{o}}(y)=d_{\mathrm{N}}(y), d_{\mathfrak{o}}(y)=a_{\mathrm{N}}(y), a_{\mathfrak{o}}(y) a_{\mathrm{N}}(y+i b)=d_{\mathfrak{o}}(y) d_{\mathrm{N}}(y-$ 
$i b)=1$. Noting that

$$
\begin{aligned}
\Psi(\mathbf{y}) & =\lim _{u \rightarrow \pm \infty}\left[e^{\pi b \mathrm{~N}(2 \kappa \pm(i b-2 u))} \operatorname{det}_{q} \mathrm{M}(u)\right] \Psi(\mathbf{y})= \\
& = \begin{cases}\mathrm{A}_{\mathfrak{o}} \mathrm{D}_{\mathfrak{o}} \Psi(\mathbf{y})=a_{\mathfrak{o}}\left(y_{\mathfrak{o}}-i b\right) d_{\mathfrak{o}}\left(y_{\mathfrak{o}}\right) \Psi(\mathbf{y}), & u \rightarrow+\infty \\
\mathrm{A}_{\mathrm{N}} \mathrm{D}_{\mathrm{N}} \Psi(\mathbf{y})=a_{\mathrm{N}}\left(y_{\mathfrak{o}}+i b\right) d_{\mathrm{N}}\left(y_{\mathfrak{o}}\right) \Psi(\mathbf{y}), & u \rightarrow-\infty\end{cases}
\end{aligned}
$$

allows us to choose

$$
a_{\mathrm{o}}=d_{\mathrm{o}}=a_{\mathrm{N}}=d_{\mathrm{N}}=1
$$

The resulting equation for the $y_{\mathrm{o}}$-dependence may therefore be written as

$$
\lim _{u \rightarrow \pm \infty}\left[( \pm)^{\mathrm{N}} e^{\pi \mathrm{N}(\kappa \mp b u)} t(u)\right] \Psi(\mathbf{y})=\left(\mathrm{T}_{\mathcal{o}}^{+}+\mathrm{T}_{\mathcal{o}}^{-}\right) \Psi(\mathbf{y})
$$

This relation supplements the Baxter equations (90) in the cases of the modular XXZ magnet and the sinh-Gordon model with even $\mathrm{N}$.

In the case of the modular XXZ magnet we furthermore find a small modification in the form of the coefficient functions $a(u)$ and $d(u)$ which appear in the Baxter equations. These follow from the following formula for the q-determinant (see Appendix C.2):

$$
\begin{aligned}
\operatorname{det}_{q} \mathrm{M}^{\mathrm{xxz}}(u) & \equiv A(u) D(u-i b)-q^{-1} B(u) C(u-i b) \\
& =\left(4 \cosh \pi b\left(s+u-i \frac{b}{2}\right) \cosh \pi b\left(s-u+i \frac{b}{2}\right)\right)^{\mathrm{N}},
\end{aligned}
$$

The resulting expressions for $a^{\mathrm{xxz}}(u)$ and $d^{\mathrm{xxz}}(u)$ will be

$$
a^{\mathrm{xxz}}(u)=d^{\mathrm{xxz}}(-u)=-2 i \cosh \pi b\left(u-s-i \frac{b}{2}\right) .
$$

The existence of the "zero mode" $y_{0}$ also leads to modifications in the formulae for $\mathrm{A}(u)$ and $\mathrm{D}(u)$. For the modular magnet and the sinh-Gordon model with even $\mathrm{N}$, the number of coefficients in the expansion (C.6) and (C.8) exceeds by two the number of the operators $\mathrm{y}_{k}$. However, in these cases we know the asymptotics of $\mathrm{A}(u)$ and $\mathrm{D}(u)$ and therefore we will need the following interpolation formula.

Lemma 2. Let $e^{\mathrm{N} \pi b u} P(u)$ be a polynomial in $e^{2 \pi b u}$ such that

$$
P(u) \sim \begin{cases}e^{+\pi b\left(\mathrm{~N} u+p_{\mathrm{o}}\right)}, & \text { for } u \rightarrow+\infty \\ (-)^{\mathrm{N}} e^{-\pi b\left(\mathrm{~N} u+p_{\mathfrak{o}}\right)}, & \text { for } u \rightarrow-\infty\end{cases}
$$

For an arbitrary set of variables $y_{1}, \ldots, y_{\mathrm{N}-1}$ such that $y_{k} \neq y_{l}$ for all $k \neq l$ we may then write

$$
P(u)=\sinh \pi b\left(u+p_{\mathcal{o}}+\rho_{\mathrm{N}}\right) \prod_{k=1}^{\mathrm{N}-1} \sinh \pi b\left(u-y_{k}\right)+\sum_{k=1}^{\mathrm{N}-1} \prod_{l \neq k} \frac{\sinh \pi b\left(u-y_{l}\right)}{\sinh \pi b\left(y_{k}-y_{l}\right)} P\left(y_{k}\right),
$$

where $\rho_{\mathrm{N}} \equiv \sum_{k=1}^{\mathrm{N}-1} y_{k}$. 
Thus, for the modular magnet and the sinh-Gordon model with even N, we have the following formulae for the operators $\mathrm{A}(u)$ and $\mathrm{D}(u)$ (recall that $\kappa=0$ for the modular magnet and $\kappa=i / 2+b s$ for the sinh-Gordon model with even $\mathrm{N}$ )

$$
\begin{array}{r}
\mathrm{A}(u)=2^{\mathrm{N}} e^{-\pi \kappa \mathrm{N}} \sinh \pi b\left(u+\mathrm{p}_{\mathrm{N}}+\rho_{\mathrm{N}}\right) \prod_{k=1}^{\mathrm{N}-1} \sinh \pi b\left(u-\mathrm{y}_{k}\right) \\
\quad+\sum_{k=1}^{\mathrm{N}-1} \prod_{l \neq k} \frac{\sinh \pi b\left(u-\mathrm{y}_{l}\right)}{\sinh \pi b\left(\mathrm{y}_{k}-\mathrm{y}_{l}\right)}\left(a\left(\mathrm{y}_{k}\right)\right)^{\mathrm{N}} \mathrm{T}_{k}^{-}, \\
\mathrm{D}(u)=2^{\mathrm{N}} e^{-\pi \kappa \mathrm{N}} \sinh \pi b\left(u+\mathrm{p}_{\mathrm{N}}+\rho_{\mathrm{N}}\right) \prod_{k=1}^{\mathrm{N}-1} \sinh \pi b\left(u-\mathrm{y}_{k}\right) \\
+\sum_{k=1}^{\mathrm{N}-1} \prod_{l \neq k} \frac{\sinh \pi b\left(u-\mathrm{y}_{l}\right)}{\sinh \pi b\left(\mathrm{y}_{k}-\mathrm{y}_{l}\right)}\left(d\left(\mathrm{y}_{k}\right)\right)^{\mathrm{N}} \mathrm{T}_{k}^{+},
\end{array}
$$

where now $\rho_{\mathrm{N}} \equiv \sum_{k=1}^{\mathrm{N}-1} \mathrm{y}_{k}$; notice that $\left[\mathrm{p}_{\mathrm{N}}, \rho_{\mathrm{N}}\right]=0$ and $\left[\mathrm{p}_{\mathrm{N}}, \mathrm{y}_{\mathrm{o}}\right]=\frac{i}{\pi b}$. Furthermore, comparision with (C.13) and (C.15) shows that $\mathrm{p}_{\mathrm{N}}=-\sum_{k=1}^{\mathrm{N}} \mathrm{p}_{k}$ for the modular magnet and $\mathrm{p}_{\mathrm{N}}=\sum_{k=1}^{\mathrm{N}}(-)^{k} \mathrm{p}_{k}$ for the sinh-Gordon model.

\section{Concluding remarks - outlook}

\subsection{On the Baxter equations}

Summarizing the results of Sections 4 and [5] we arrive at the main result of the present article. We will formulate it only for the case of the sinh-Gordon model with $\mathrm{N}$ odd for which our analysis is most complete, but from our previous discussions and remarks it seems clear that very similar results should hold in the other cases as well.

Main result: A function $t(u)$ is an eigenvalue of the transfer-matrix $\mathrm{T}^{\mathrm{SGo}}(u)$ if and only if there exists a function $q_{t}(u)$ which satisfies the following conditions

(i) $q_{t}(u)$ is meromorphic in $\mathbb{C}$, with poles of maximal order $\mathrm{N}$ in $\Upsilon_{-s} \cup \bar{\Upsilon}_{s}$,

(ii) $q_{t}(u) \sim\left\{\begin{array}{lll}\exp \left(+i \pi \mathrm{N} \sigma u-i \frac{\pi}{2} \mathrm{~N} u^{2}\right) & \text { for }|u| \rightarrow \infty, & |\arg (u)|<\frac{\pi}{2}, \\ \exp \left(-i \pi \mathrm{N} \sigma u-i \frac{\pi}{2} \mathrm{~N} u^{2}\right) & \text { for }|u| \rightarrow \infty, \quad|\arg (u)|>\frac{\pi}{2}\end{array}\right.$

(iii) $t(u) q_{t}(u)=(\check{a}(u))^{\mathrm{N}} q_{t}(u-i b)+(\check{d}(u))^{\mathrm{N}} q_{t}(u+i b)$,

where $\check{d}(u)=\check{a}(-u)=1+\left(\frac{m \Delta}{4}\right)^{2} e^{-\pi b(2 u+i b)}$,

(iv) $q_{t}(u)$ satisfies the following quantum Wronskian relation

$$
q_{t}\left(u+i \delta_{+}\right) q_{t}\left(u-i \delta_{+}\right)-q_{t}\left(u+i \delta_{-}\right) q_{t}\left(u-i \delta_{-}\right)=W_{\mathrm{N}}(u),
$$

where $W_{\mathrm{N}}(u)=e^{-i \pi \mathrm{N}\left(u^{2}+\sigma^{2}\right)}\left(D_{\sigma}(u)\right)^{-\mathrm{N}}$.

The corresponding eigenstate $\Psi_{t}$ in the SOV representation defined in Section 5 is represented as in (92).

We have therefore succeeded in reformulating the spectral problem for $\mathrm{T}(u)$ as the problem to determine the set $\mathfrak{S}$ of solutions to the Baxter equation which possess the properties (i)-(iv) above. 
It should be observed that conditions (i)-(iii) already constrain the possible functions $q_{t}(u)$ rather strongly. Let us consider

$$
Q_{t}(u)=\left(\Gamma_{b}\left(\frac{Q}{2}-i(u+s)\right) \Gamma_{b}\left(\frac{Q}{2}-i(u-s)\right)\right)^{-\mathrm{N}} q_{t}(u),
$$

where $\Gamma_{b}(x) \equiv \Gamma_{2}\left(x \mid b^{-1}, b\right)$, with $\Gamma_{2}$ being the Barnes Double Gamma function defined in Appendix A.1 The function $Q_{t}(u)$ will then have the properties

$$
\left[\begin{array}{l}
\text { (a) } Q_{t}(u) \text { is entire analytic of order } 2 \text { in } \mathbb{C} \\
\text { (b) } t(u) Q_{t}(u)=(A(u))^{\mathrm{N}} Q_{t}(u-i b)+(D(u))^{\mathrm{N}} Q_{t}(u+i b),
\end{array}\right]
$$

The explicit form of the coefficients $A(u)$ and $D(u)$ can easily be figured out with the help of the formula 69, and the functional relations A.4.

Property (a) combined with the Hadamard factorization theorem (see e.g. [Ti]) imply that $Q_{t}(u)$ can be represented by a product representation of the form

$$
Q_{t}(u)=e^{r(u)} \prod_{k=1}^{\infty}\left(1-\frac{u}{u_{k}}\right)
$$

where the prime indicates the canonical Weierstrass regularization of the infinite product. The function $r(u)$ in the prefactor is a second order polynomial which can be worked out explicitly. The Baxter equation (57) then implies that the zeros $u_{k}$ must satisfy an infinite set of equations,

$$
-1=\frac{\left(A\left(u_{k}\right)\right)^{\mathrm{N}}}{\left(D\left(u_{k}\right)\right)^{\mathrm{N}}} \frac{Q_{t}\left(u_{k}-i b\right)}{Q_{t}\left(u_{k}+i b\right)}, \quad k \in \mathbb{N},
$$

which may be regarded as a generalization of the Bethe ansatz equations. However, as it stands it is not quite clear if these equations represent an efficient starting point for the investigation of the spectrum of our models.

The quantum Wronskian relation (iv) encodes remarkable additional information which can not easily be extracted from the conditions (i)-(iii) above. We plan to discuss its implications elsewhere.

\subsection{Continuum limit}

It is certainly interesting to discuss the consistency of our results with existing results and conjectures on the sinh-Gordon model in continuous space-time. Let us therefore now show that our findings are consistent with Lukyanov's remarkable conjecture [ $[\mathrm{Lu}]$ on the ground state wavefunction for the sinh-Gordon model in the SOV representation.

Recall from the Subsection 3.3 that we are interested in the limit $N \rightarrow \infty, \Delta \rightarrow 0, s \rightarrow \infty$ such that $m=\frac{4}{\Delta} e^{-\pi b s}$ and $R=\mathrm{N} \Delta / 2 \pi$ are kept finite in the limit. We are interested in the limiting behavior of the Baxter equation and of its solutions. Let us first note that the poles of $q_{t}(u)$ move out to infinity when $s \rightarrow \infty$. Also note that according to property (129-ii) rapid decay is found only within the strip $\mathcal{S}=\{u \in \mathbb{C} ;|\operatorname{Im}(u)|<Q / 2\}$. By noting that $m \Delta=\mathcal{O}(1 / N)$ in the limit under consideration one sees that the coefficients $\check{a}(u), \breve{d}(u)$ in the Baxter equation (129-iii) become unity when $\mathrm{N} \rightarrow \infty$. Most importantly, let us finally observe that the right hand side of the Wronskian relation (129-iv) approaches a constant for $\mathrm{N} \rightarrow \infty$. 
Our results therefore strongly suggest the following conjecture on the conditions which characterize the spectrum of the continuum sinh-Gordon model in the SOV representation:

(i) $q_{t}(u)$ is entire analytic,

(ii) $q_{t}(u)$ decays rapidly for $|\operatorname{Re}(u)| \rightarrow \infty, u \in \mathcal{S}$,

(iii) $q_{t}(u)$ satisfies a difference equation of the form

$$
t(u) q_{t}(u)=q_{t}(u-i b)+q_{t}(u+i b)
$$

where $t(u)$ is periodic under $u \rightarrow u+i b^{-1}$,

(iv) $q_{t}(u)$ satisfies the following quantum Wronskian relation

$$
q_{t}\left(u+i \delta_{+}\right) q_{t}\left(u-i \delta_{+}\right)-q_{t}\left(u+i \delta_{-}\right) q_{t}\left(u-i \delta_{-}\right)=1 .
$$

Our next aim will be to show that, by adding one supplementary condition, one gets a complete characterization of the function $q_{0}(u)$ which was proposed in [Lu] to describe the ground state for the continuum sinh-Gordon model in the SOV representation:

(v) $\quad q_{0}(u)$ is nonvanishing within $\mathcal{S}$.

We claim that the solution to conditions (i)-(v) is essentially unique and given by the formula

$$
\log q_{0}(u)=-\frac{m R}{2} \frac{\cosh \frac{\pi}{Q} u}{\sin \frac{\pi}{Q} b}+\int_{\mathbb{R}} \frac{d v}{2 Q} \frac{\log (1+Y(v))}{\cosh \frac{\pi}{Q}(u-v)},
$$

which expresses $q_{0}(u)$ in terms of the solution $Y(u)$ to the nonlinear integral equation

$$
\log Y(u)=-m R \cosh \frac{\pi}{Q} u+\int_{\mathbb{R}} \frac{d v}{2 Q} S(u-v) \log (1+Y(v)),
$$

where the kernel $S(u-v)$ is explicitly given as follows:

$$
S(u)=\frac{2 \sin \frac{\pi}{Q} b \cosh \frac{\pi}{Q} u}{\sinh \frac{\pi}{Q}(u+i b) \sinh \frac{\pi}{Q}(u-i b)} .
$$

These equations form the basis for the calculation of the ground state energy [Za $]$ and other local conserved quantities of the continuum sinh-Gordon model $[\mathrm{Lu}]$ within the thermodynamic Bethe ansatz framework.

For the reader's convenience we will present the outline of an argument ${ }^{4}$ which establishes the equivalence between (i)-(v) and (136), (137). Let us define an auxilliary function $Y(u)$ by the formula

$$
1+Y(u)=q_{0}\left(u+i \delta_{+}\right) q_{0}\left(u-i \delta_{+}\right) .
$$

Assuming that $q_{0}(u)$ satisfies the properties (i), (ii), and (v), one can take the logarithm of (139) and then solve the resulting difference equation by Fourier transform, which leads to the representation (136). Re-inserting this representation into the Wronskian relation (iv) shows that $Y(u)$ must satisfy 137 .

A proof of Lukyanov's conjecture $[\overline{\mathrm{Lu}}]$ therefore amounts to showing that $q_{0}(u)$ must satisfy the properties (i)-(v). We find it very encouraging that our study of the lattice sinh-Gordon model gave us strong support for the necessity of properties (i)-(iv).

\footnotetext{
${ }^{4}$ This argument is inspired by the considerations in [Za] and a suggestion of F. Smirnov (private communication, see also [Sm2]). However, the key point of our argument, namely the origin of the quantum Wronskian relation (iv) seems to be new.
} 


\subsection{Connection with lattice Liouville model}

Relations between the compact XXZ chain, lattice sine-Gordon model, and the (imaginary field) Liouville model was investigated in $[\mathrm{FT}]$ from the view point of the QISM. Let us show that similar connections exist between the modular XXZ magent, lattice sinh-Gordon model, and the (real field) Liouville model. Following [FT], we introduce the L-matrix

$$
L^{\zeta}(u)=e^{-\frac{1}{2} \pi b \zeta \sigma_{3}} L^{\mathrm{SG}}(u+\zeta) e^{\frac{1}{2} \pi b \zeta \sigma_{3}},
$$

where $\zeta$ is related to the representation parameter $s$ and the lattice spacing $\Delta$ as $e^{\pi b \zeta}=\Delta e^{\pi b s}$, and the operators $p$ and $x$ in (33) are related to the discretized field and its conjugate momentum as follows

$$
2 \pi b \mathrm{p}=2 \pi b \zeta-\beta \Phi, \quad 4 \pi b \mathrm{x}=2 \pi b \zeta+\beta\left(\frac{1}{2} \Pi-\Phi\right), \quad \beta=b \sqrt{8 \pi} .
$$

Comparison with (47) and (49) shows that the new variables defined by (141) are related to these of the sinh-Gordon model via a canonical transformation,

$$
\Pi=\Pi^{\mathrm{SG}}, \quad \beta \Phi=\beta \Phi^{\mathrm{SG}}+2 \pi b \zeta, \quad e^{-\pi b \zeta}=\frac{m}{4} .
$$

In the $\zeta \rightarrow+\infty$ limit L-matrix (140) turns into

$$
L^{\mathrm{L}}(u) \equiv \lim _{\zeta \rightarrow+\infty} L^{\zeta}(u)=\left(\begin{array}{cc}
e^{\frac{\beta}{8} \Pi_{n}}\left(1+\Delta^{2} e^{-\beta \Phi_{n}}\right) e^{\frac{\beta}{8} \Pi_{n}} & -i \Delta e^{\pi b u-\frac{\beta}{2} \Phi_{n}} \\
-2 i \Delta \sinh \left(\pi b u+\frac{\beta}{2} \Phi_{n}\right) & e^{-\frac{\beta}{4} \Pi_{n}}
\end{array}\right) .
$$

It is natural to expect that this L-matrix describes some massless limit of the sinh-Gordon model. The corresponding U-matrix obtained according to formula (52),

$$
U^{\mathrm{L}}(u)=\left(\begin{array}{cc}
\frac{\beta}{4} \Pi(x) & -i e^{u-\frac{\beta}{2} \Phi(x)} \\
-2 i \sinh \left(u+\frac{\beta}{2} \Phi(x)\right) & -\frac{\beta}{4} \Pi(x)
\end{array}\right),
$$

reproduces the Liouville equations of motion via the zero curvature equation (see [FT] for details in the case of sine-Gordon model and imaginary Liouville field). This observation suggests that (143) is a suitable L-matrix for describing the quantum lattice Liouville model in the QISM framework.

Although the limiting procedure in 143 has not been mathematically rigorously developed yet (in particular, there is a subtle question of interchangibility of $\zeta \rightarrow+\infty$ limit with the classical limit), the results of the present article provide further support for the proposed connection between the sinh-Gordon and Liouville models. First, observe that the twist by $e^{\frac{1}{2} \pi b \zeta \sigma_{3}}$ and the shift of the spectral parameter in (140) do not change the corresponding auxiliary R-matrix (34) and the fundamental R-matrix given in Proposition 1 Therefore, the local lattice density of the classical Hamiltonian corresponding to (140) can be obtained by substituting (142) into (46). Taking then the limit $\zeta \rightarrow+\infty$, we obtain the following lattice Hamiltonian density (up to an additive constant)

$$
\begin{aligned}
H_{n, n+1}^{\mathrm{L}, \mathrm{cl}} & \equiv \lim _{\zeta \rightarrow \infty} H_{n, n+1}^{\mathrm{SG}, \mathrm{cl}}=\frac{1}{\gamma} \log \left(\frac{1}{2} \cosh \frac{\beta}{4}\left(\Pi_{n}+\Pi_{n+1}\right)+\frac{1}{2} \cosh \frac{\beta}{2}\left(\Phi_{n}-\Phi_{n+1}\right)\right. \\
& \left.+\frac{\Delta^{2}}{2} e^{-\frac{\beta}{2}\left(\Phi_{n}+\Phi_{n+1}\right)}\left(1+e^{\frac{\beta}{4}\left(\Pi_{n}+\Pi_{n+1}\right)} \cosh \frac{\beta}{2}\left(\Phi_{n}-\Phi_{n+1}\right)\right)\right)
\end{aligned}
$$


which in the continuum limit (50) yields the Liouville Hamiltonian:

$$
\sum_{n} \frac{1}{\Delta} H_{n, n+1}^{\mathrm{L}, \mathrm{cl}} \rightarrow \mathrm{const}+\int_{0}^{2 \pi R} d x\left(\frac{1}{2} \Pi^{2}+\frac{1}{2}\left(\partial_{x} \Phi\right)^{2}+\frac{1}{\gamma} e^{-\beta \Phi}\right)
$$

The second observation that we can make to support the proposed relationship between the lattice sinh-Gordon and Liouville models is the following. The transfer-matrix corresponding to (140) is given by $\mathrm{T}^{\zeta}(u)=\mathrm{T}^{\mathrm{SG}}(u+\zeta)$. Therefore, $\mathrm{Q}_{ \pm}^{\zeta}(u) \equiv \check{Q}_{ \pm}^{\mathrm{SG}}(u+\zeta)$ satisfy (cf. (79)) the Baxter equation

$$
\begin{aligned}
& \mathrm{T}^{\zeta}(u) \cdot \mathrm{Q}_{ \pm}^{\zeta}(u)=\left(a_{\zeta}(u)\right)^{\mathrm{N}} \mathrm{Q}_{ \pm}^{\zeta}(u-i b)+\left(d_{\zeta}(u)\right)^{\mathrm{N}} \mathrm{Q}_{ \pm}^{\zeta}(u+i b), \\
& \text { where } \quad a_{\zeta}(u)=1+\Delta^{2} e^{\pi b(2 u-i b)}, \quad d_{\zeta}(u)=1+\Delta^{2} e^{-\pi b(2 u+4 \zeta+i b)} .
\end{aligned}
$$

Hence for $\mathrm{T}^{\mathrm{L}}(u) \equiv \lim _{\zeta \rightarrow+\infty} \mathrm{T}^{\zeta}(u)$ and $\mathrm{Q}_{ \pm}^{\mathrm{L}}(u) \equiv \lim _{\zeta \rightarrow+\infty} \mathrm{Q}_{ \pm}^{\zeta}(u)$ (the limits are meaningful if $\mathrm{T}^{\zeta}(u)$ and $\mathrm{Q}_{ \pm}^{\zeta}(u)$ are expressed in terms of $\Phi$ and $\left.\Pi\right)$, we obtain the Baxter equation

$$
\mathrm{T}^{\mathrm{L}}(u) \cdot \mathrm{Q}_{ \pm}^{\mathrm{L}}(u)=\left(1+\Delta^{2} e^{\pi b(2 u-i b)}\right)^{\mathrm{N}} \mathrm{Q}_{ \pm}^{\mathrm{L}}(u-i b)+\mathrm{Q}_{ \pm}^{\mathrm{L}}(u+i b)
$$

which coincides ${ }^{5}$ with the Baxter equation derived for the lattice Liouville model by a different method in [K1] FKV].

We finish by noting that in the continuum limit, $N \rightarrow \infty, \Delta=\mathcal{O}(1 / N)$, the coefficient $\left(1+\Delta^{2} e^{\pi b(2 u-i b)}\right)^{\mathrm{N}}$ becomes unity. This suggests that the Baxter equation for the eigenvalue $q_{t}^{\mathrm{L}}(u)$ of the $\mathrm{Q}-$ operator for the continuum Liouville model is

$$
t^{\mathrm{L}}(u) q_{t}^{\mathrm{L}}(u)=q_{t}^{\mathrm{L}}(u-i b)+q_{t}^{\mathrm{L}}(u+i b), \quad t^{\mathrm{L}}\left(u+i b^{-1}\right)=t^{\mathrm{L}}(u),
$$

which coincides with that for the continuum sinh-Gordon model (134-iii).

However, it seems to be crucial to observe that the asymptotic properties of the function $q_{t}^{\mathrm{L}}(u)$ will certainly differ from those found in the case of the sinh-Gordon model. Indeed, for any model, the asymptotic properties of $q_{t}(u)$ are related to these of $t(u)$. Comparing the structure of the L-matrices (33) and (143), we see that the transfer-matrix $\mathrm{T}^{\mathrm{L}}(u)$ corresponding to the latter L-matrix has asymmetric asymptotics for $\operatorname{Re}(u) \rightarrow \pm \infty$. This seems to be related to the fact that the sinh-Gordon potential $\cosh \beta \Phi(x)$ is spatially symmetric while the Liouville potential $e^{-\beta \Phi(x)}$ is asymmetric. As a consequence, we expect that the set of solutions to the Baxter equation (149) describing the spectrum of the continuum Liouville model will be quite different from the set of solutions to the same Baxter equation which characterizes the spectrum of the continuum sinhGordon model.

These observations seem to offer a key to the understanding of the relation between massive and massless theories from the point of view of their integrable structure.

\footnotetext{
${ }^{5}$ modulo notations, in particular, $\Delta=1$ in $[\mathrm{K} 1$ FKV]
} 


\section{A Special functions}

\section{A.1 Double Gamma function}

All the special functions that we have to deal with can be obtained from the Barnes Double Gamma function $\Gamma_{2}\left(x \mid \omega_{1}, \omega_{2}\right)[\mathrm{Ba}]$, which may be defined by

$$
\log \Gamma_{2}\left(x \mid \omega_{1}, \omega_{2}\right)=\left(\frac{\partial}{\partial t} \sum_{n_{1}, n_{2}=0}^{\infty}\left(x+n_{1} \omega_{1}+n_{2} \omega_{2}\right)^{-t}\right)_{t=0} .
$$

The infinite sum in (A.1) is defined by analytic continuation from its domain of convergence $(\operatorname{Re}(t)>2)$ to the point of interest $(t=0)$. One may alternatively use the integral representation

$$
\log \Gamma_{2}\left(x \mid \omega_{1}, \omega_{2}\right)=\frac{C}{2} B_{2,2}\left(x \mid \omega_{1}, \omega_{2}\right)+\frac{1}{2 \pi i} \int_{\mathcal{C}} \frac{e^{-x t} \log (-t)}{\left(1-e^{-\omega_{1} t}\right)\left(1-e^{-\omega_{2} t}\right)} \frac{d t}{t}
$$

where the contour $\mathcal{C}$ goes from $+\infty$ to $+\infty$ encircling 0 counterclockwise, $C$ is the Euler's constant and

$$
B_{2,2}\left(x \mid \omega_{1}, \omega_{2}\right)=\frac{\left(2 x-\omega_{1}-\omega_{2}\right)^{2}}{4 \omega_{1} \omega_{2}}-\frac{\omega_{1}^{2}+\omega_{2}^{2}}{12 \omega_{1} \omega_{2}}
$$

The integral is well defined if $\operatorname{Re}\left(\omega_{1}\right)>0, \operatorname{Re}\left(\omega_{2}\right)>0$, and $\operatorname{Re}(x)>0$. It satisfies the basic functional relations

$$
\frac{\Gamma_{2}\left(x+\omega_{1} \mid \omega_{1}, \omega_{2}\right)}{\Gamma_{2}\left(x \mid \omega_{1}, \omega_{2}\right)}=\sqrt{2 \pi} \frac{\omega_{2}^{\frac{1}{2}-\frac{x}{\omega_{2}}}}{\Gamma\left(x / \omega_{2}\right)}, \quad \frac{\Gamma_{2}\left(x+\omega_{2} \mid \omega_{1}, \omega_{2}\right)}{\Gamma_{2}\left(x \mid \omega_{1}, \omega_{2}\right)}=\sqrt{2 \pi} \frac{\omega_{1}^{\frac{1}{2}-\frac{x}{\omega_{1}}}}{\Gamma\left(x / \omega_{1}\right)} .
$$

$\left(\Gamma_{2}\left(x \mid \omega_{1}, \omega_{2}\right)\right)^{-1}$ is an entire analytic function of order 2 w.r.t. its variable $x$ with simple zeros at $x=-m \omega_{1}-n \omega_{2}$, where $m$ and $n$ are non-negative integers.

\section{A.2 Function $w_{b}(x)$}

In what follows we will be dealing with

$$
w_{b}(x) \equiv \frac{\Gamma_{2}\left(\frac{Q}{2}-i x \mid b^{-1}, b\right)}{\Gamma_{2}\left(\frac{Q}{2}+i x \mid b^{-1}, b\right)} .
$$

In the strip $|\operatorname{Im}(x)|<\frac{Q}{2}$, function $w_{b}(x)$ has the following integral representation

$$
w_{b}(x)=\exp \left\{\frac{i \pi}{2} x^{2}+\frac{i \pi}{24}\left(b^{2}+b^{-2}\right)-\int_{\mathbb{R}+i 0} \frac{d t}{4 t} \frac{e^{-2 i t x}}{\sinh b t \sinh \frac{t}{b}}\right\}
$$

where the integration contour goes around the pole $t=0$ in the upper half-plane. This function is closely related (cf. Eq. (D.7)) to the remarkable special function introduced under the name of quantum dilogarithm in [FK2] and studied in the context of quantum groups and integrable models in [F2] Ru Wo PT2 K1 K2, BT] T2, I2]. 
Analytic continuation of $w_{b}(x)$ to the entire complex plane is a meromorphic function with the following properties

$$
\begin{aligned}
& \text { self-duality } \quad w_{b}(x)=w_{b^{-1}}(x), \\
& \text { functional equation } \frac{w_{b}\left(x+\frac{i}{2} b^{ \pm 1}\right)}{w_{b}\left(x-\frac{i}{2} b^{ \pm 1}\right)}=2 \cosh \left(\pi b^{ \pm 1} x\right) \text {, } \\
& \text { reflection property } w_{b}(x) w_{b}(-x)=1, \\
& \text { complex conjugation } \overline{w_{b}(x)}=w_{b}(-\bar{x}) \text {, } \\
& \text { zeros / poles }\left(w_{b}(x)\right)^{ \pm 1}=0 \Leftrightarrow \pm x \in\left\{i \frac{Q}{2}+n b+m b^{-1} ; n, m \in \mathbb{Z}^{\geq 0}\right\}, \\
& \text { residue } \operatorname{Res}_{x=-i \frac{Q}{2}} w_{b}(x)=\frac{i}{2 \pi} \\
& \text { asymptotics } \quad w_{b}(x) \sim \begin{cases}e^{-\frac{i \pi}{2}\left(x^{2}+\frac{1}{12}\left(b^{2}+b^{-2}\right)\right)} & \text { for }|u| \rightarrow \infty,|\arg (u)|<\frac{\pi}{2}, \\
e^{+\frac{i \pi}{2}\left(x^{2}+\frac{1}{12}\left(b^{2}+b^{-2}\right)\right)} \text { for }|u| \rightarrow \infty,|\arg (u)|>\frac{\pi}{2} .\end{cases}
\end{aligned}
$$

Notice that $\left|w_{b}(x)\right|=1$ if $x \in \mathbb{R}$. Therefore, $w_{b}(\mathrm{O})$ is unitary if $\mathrm{O}$ is a self-adjoint operator.

The function $w_{b}(x)$ allows us to define a whole class of new special functions. In Appendix D we will use in particular the following b-analogues of the hypergeometric functions defined by

$$
\Phi_{r}\left(U_{1} \ldots U_{r} ; V_{1} \ldots V_{r} ; x\right) \equiv \frac{1}{i} \int_{i \mathbb{R}-0} d \tau e^{\pi \tau x} \prod_{k=1}^{r} \frac{S_{b}\left(U_{k}+\tau\right)}{S_{b}\left(V_{k}+\tau\right)}
$$

where the special function $S_{b}(x)$ is defined by

$$
S_{b}(x)=w_{b}\left(i x-\frac{i}{2} Q\right)
$$

and has the properties

$$
\begin{aligned}
\text { self-duality } & S_{b}(x)=S_{b^{-1}}(x), \\
\text { functional equation } & S_{b}\left(x+b^{ \pm 1}\right)=2 \sin \left(\pi b^{ \pm 1} x\right) S_{b}(x), \\
\text { reflection property } & S_{b}(x) S_{b}(Q-x)=1 .
\end{aligned}
$$

\section{A.3 Function $D_{\alpha}(x)$}

Let us also introduce another useful function

$$
D_{\alpha}(x)=\frac{w_{b}(x+\alpha)}{w_{b}(x-\alpha)} .
$$

Combining A.6 with A.22, we derive the integral representation

$$
D_{\alpha}(x)=\exp \left\{i \int_{\mathbb{R}+i 0} \frac{d t}{2 t} \frac{\cos (2 t x) \sin (2 \alpha t)}{\sinh b t \sinh \frac{t}{b}}\right\} .
$$


$D_{\alpha}(x)$ is a meromorphic function with zeros at $\pm x \in \Upsilon_{-\alpha}$ and poles at $\pm x \in \Upsilon_{\alpha}$, where the set $\Upsilon_{\alpha}$ is defined in B.1. The function $D_{\alpha}(x)$ is self-dual in $b$ (but we will omit this index) and has the following properties

$$
\begin{aligned}
& \text { functional equation } \frac{D_{\alpha}\left(x+\frac{i}{2} b^{ \pm 1}\right)}{D_{\alpha}\left(x-\frac{i}{2} b^{ \pm 1}\right)}=\frac{\cosh \pi b^{ \pm 1}(x+\alpha)}{\cosh \pi b^{ \pm 1}(x-\alpha)}, \\
& x \text {-parity } D_{\alpha}(x)=D_{\alpha}(-x), \\
& \text { reflection property } \frac{D_{\alpha}(x) D_{-\alpha}(x)=1,}{D_{\alpha}(x)}=D_{-\bar{\alpha}}(\bar{x}), \\
& \text { complex conjugation } \\
& x \text {-asymptotics } \quad D_{\alpha}(x) \sim \begin{cases}e^{-2 \pi i \alpha x} \text { for }|u| \rightarrow \infty,|\arg (u)|<\frac{\pi}{2}, \\
e^{+2 \pi i \alpha x} \text { for }|u| \rightarrow \infty,|\arg (u)|>\frac{\pi}{2},\end{cases} \\
& \qquad \text {-asymptotics } \quad D_{\alpha}(x) \sim \begin{cases}e^{-i \pi\left(x^{2}+\alpha^{2}+\frac{1}{12}\left(b^{2}+b^{-2}\right)\right)} \text { if }|u| \rightarrow \infty,|\arg (u)|<\frac{\pi}{2}, \\
e^{+i \pi\left(x^{2}+\alpha^{2}+\frac{1}{12}\left(b^{2}+b^{-2}\right)\right)} \text { if }|u| \rightarrow \infty,|\arg (u)|>\frac{\pi}{2} .\end{cases}
\end{aligned}
$$

Also, the following identity is obvious from the definition A.19

$$
D_{\alpha}(x) D_{\beta}(y)=D_{\frac{\alpha+\beta+x-y}{2}}\left(\frac{x+y+\alpha-\beta}{2}\right) D_{\frac{\alpha+\beta-x+y}{2}}\left(\frac{x+y-\alpha+\beta}{2}\right) .
$$

Notice that $\left|D_{\alpha}(x)\right|=1$ if $\alpha \in \mathbb{R}$ and $x \in \mathbb{R}$ or $x \in i \mathbb{R}$. Therefore, $D_{\alpha}(\mathrm{O})$ is unitary if $\alpha \in \mathbb{R}$ and $\mathrm{O}$ is a self-adjoint or anti-self-adjoint operator.

\section{A.4 Integral identities for $D_{\alpha}(x)$}

Here we will give some integral identities involving products of D-functions. These identities can be regarded as summation formulae for the b-hypergeometric functions $\Phi_{r}$ introduced in (A.14).

Let us denote $\alpha^{\star} \equiv-\frac{i}{2} Q-\alpha$ and introduce the function

$$
A\left(\alpha_{1}, \alpha_{2}, \ldots\right)=w_{b}\left(\alpha_{1}-\alpha_{1}^{\star}\right) w_{b}\left(\alpha_{2}-\alpha_{2}^{\star}\right) \ldots
$$

Notice that $\left(\alpha^{\star}\right)^{\star}=\alpha$ and hence $A\left(\alpha_{1}^{\star}, \alpha_{2}^{\star}, \ldots\right) A\left(\alpha_{1}, \alpha_{2}, \ldots\right)=1$.

Lemma 15 in [PT2] and Eqs. (26)-(27) in [FKV] can be rewritten as the following property of the function $D_{\alpha}(x)$ under the Fourier transform:

$$
\int_{\mathbb{R}} d x e^{2 \pi i x y} D_{\alpha}(x)=A(\alpha) D_{\alpha^{\star}}(y)
$$

Taking into account that $\lim _{\alpha \rightarrow 0} D_{\alpha}(x)=1$, we obtain from A.29) as a special case

$$
\lim _{\alpha \rightarrow-\frac{i}{2} Q} A\left(\alpha^{\star}\right) D_{\alpha}(x)=\delta(x)
$$

Here $\delta(x)$ on the r.h.s. is the Dirac delta-function and this relation should be understood in the sense of distributions. Indeed $\lim _{\alpha \rightarrow-\frac{i}{2} Q} A\left(\alpha^{\star}\right)=w_{b}\left(\frac{i}{2} Q\right)=0$ and the 1.h.s. of A.30 vanishes almost everywhere. On the other hand, the only double pole of $D_{-\frac{i}{2} Q}(x)$ is at $x=0$. 
Using A.29, it is easy to derive the following relation

$$
\begin{aligned}
& \int_{\mathbb{R}} d x e^{2 \pi i z x} D_{\alpha}(x-u) D_{\beta}(x-v)= \\
&=A(\alpha, \beta) e^{\pi i z(u+v)} \int_{\mathbb{R}} d y e^{2 \pi i y(u-v)} D_{\alpha^{\star}}\left(y+\frac{z}{2}\right) D_{\beta^{\star}}\left(y-\frac{z}{2}\right) .
\end{aligned}
$$

Choosing $z=\alpha^{\star}+\beta^{\star}$, we can use A.27) in order to rewrite the product of $D$ 's in the integrand on the r.h.s. as a single function, $D_{\alpha^{\star}+\beta^{\star}}\left(y+\frac{\alpha^{\star}-\beta^{\star}}{2}\right)$, and then apply A.29. This yields

$$
\begin{aligned}
\int_{\mathbb{R}} d x & e^{2 \pi i\left(\alpha^{\star}+\beta^{\star}\right) x} D_{\alpha}(x-u) D_{\beta}(x-v)= \\
& =A\left(\alpha, \beta, \alpha^{\star}+\beta^{\star}\right) e^{2 \pi i\left(v \alpha^{\star}+u \beta^{\star}\right)} D_{\alpha+\beta+\frac{i}{2} Q}(u-v) .
\end{aligned}
$$

In the case $\alpha^{\star}=-\beta^{\star}$, Eq. A.30 can be used and we conclude that

$$
\int_{\mathbb{R}} d x D_{\alpha}(x-u) D_{\beta}(x-v)=A(\alpha, \beta) \delta(u-v)
$$

holds in the sense of distributions provided that $\alpha+\beta=-i Q$.

Lemma 3. The identities

$$
\begin{aligned}
& \int_{\mathbb{R}} d x D_{\alpha}(x-u) D_{\beta}(x-v) D_{\gamma}(x-w) \\
& =A(\alpha, \beta, \gamma) D_{\alpha^{\star}}(w-v) D_{\beta^{\star}}(u-w) D_{\gamma^{\star}}(v-u), \\
& \int_{\mathbb{R}} d x D_{\alpha}(x-u) D_{\beta}(x-v) D_{\gamma}(x-w) D_{\omega}(x-z) \\
& =A(\alpha, \beta, \gamma, \omega) \frac{D_{\alpha+\beta+\frac{i}{2} Q}(u-v)}{D_{\alpha+\beta+\frac{i}{2} Q}(w-z)} \int_{\mathbb{R}} d x D_{\alpha^{\star}}(x-v) D_{\beta^{\star}}(x-u) D_{\gamma^{\star}}(x-z) D_{\omega^{\star}}(x-w)
\end{aligned}
$$

are valid provided that $\alpha+\beta+\gamma=-i Q$ in (A.34), and $\alpha+\beta+\gamma+\omega=-i Q$ in (A.35).

Proof. Relation (A.34) follows straightforwardly from Eq. (19) in [K2], where function $\varphi_{b}(x)$ is our $g_{b}\left(e^{2 \pi b x}\right)$ (cf. Eq. (D.7)). Also, in other notations, relation (A.34) is Eq. (11) in [V2].

Eq. (A.34 provides two expressions for a function which we denote as $I(u, v, w ; \alpha, \beta, \gamma)$. In order to prove A.35 we multiply two copies of A.34 and compute the following integral

$$
\int_{\mathbb{R}} d t I(t, u, v ; \nu, \alpha, \beta) I(t, w, z ; \mu, \gamma, \omega),
$$

where $\nu+\alpha+\beta=\mu+\gamma+\omega=-i Q$ and we impose an additional condition $\nu+\mu=-i Q$. Then the 1.h.s. of A.35 is obtained if we substitute for the $I$ 's the expressions on the 1.h.s. of A.34 and use relation A.33). The r.h.s. of A.35 is obtained directly from A.36 if we substitute for the $I$ 's the expressions on the r.h.s. of A.34 and use also that $\mu^{\star}=-\nu^{\star}$.

\section{B Positivity versus self-duality of the representations $\mathcal{P}_{s}$}

The representations $\mathcal{P}_{s}$ are distinguished by the property that the operators $\pi_{s}(u), u \in\{E, F, K\}$ are positive self-adjoint. We are now going to show that this property is closely related to the 
remarkable self-duality of these representations under $b \rightarrow b^{-1}$ which has such profound consequences for the physics of our models.

To begin with, let us remark that there exists a linear basis $\mathcal{B}_{q}(\mathfrak{s l}(2, \mathbb{R}))$ for $\mathcal{U}_{q}(\mathfrak{s l}(2, \mathbb{R}))$ such that all elements $u$ of $\mathcal{B}_{q}(\mathfrak{s l}(2, \mathbb{R}))$ are realized by positive operators $\pi_{s}(u)$. Such a basis is, e.g., given by the monomials

$$
\begin{aligned}
& q^{+\frac{m n}{2}} C^{l} E^{m} K^{n} \text { represented by } \mathrm{C}_{s}^{l}\left(\mathrm{~K}_{s}\right)^{\frac{n}{2}}\left(\mathrm{E}_{s}\right)^{m}\left(\mathrm{~K}_{s}\right)^{\frac{n}{2}}, \\
& q^{-\frac{m n}{2}} C^{l} F^{m} K^{n} \text { represented by } \mathrm{C}_{s}^{l}\left(\mathrm{~K}_{s}\right)^{\frac{n}{2}}\left(\mathrm{~F}_{s}\right)^{m}\left(\mathrm{~K}_{s}\right)^{\frac{n}{2}},
\end{aligned}
$$

The elements of $\mathcal{U}_{q}(\mathfrak{s l}(2, \mathbb{R}))$ are clearly realized by unbounded operators on $L^{2}(\mathbb{R})$. It is therefore useful to consider suitable subspaces $\mathcal{T}_{s} \subset L^{2}(\mathbb{R})$ of test-functions on which all operators $\pi_{s}(u), u \in \mathcal{U}_{q}(\mathfrak{s l}(2, \mathbb{R}))$ are well-defined. In order to describe a canonical choice for $\mathcal{T}_{s}$ let us represent the elements of $\mathcal{T}_{s}$ by functions $f(k)$ such that $\mathrm{p}$ acts as $(\mathrm{p} f)(k)=k f(k)$.

Definition 5. Let $\mathcal{T}_{s}$ be the space of functions $f(k)$ which satisfy $e^{a|k|} f \in L^{2}(\mathbb{R})$ for all $a>0$, and which have an analytic continuation to $\mathbb{C} \backslash\left(\Upsilon_{s} \cup \bar{\Upsilon}_{-s}\right)$, where

$$
\begin{array}{ll}
\Upsilon_{s}=\left\{s+i\left(\frac{Q}{2}+n b+m b^{-1}\right),\right. & \left.n, m \in \mathbb{Z}^{\geq 0}\right\}, \\
\bar{\Upsilon}_{s}=\left\{s-i\left(\frac{Q}{2}+n b+m b^{-1}\right),\right. & \left.n, m \in \mathbb{Z}^{\geq 0}\right\} .
\end{array}
$$

On the spaces $\mathcal{T}_{s}$ the action of $\mathcal{U}_{q}(\mathfrak{s l}(2, \mathbb{R}))$ is given by

$$
\begin{aligned}
& \mathrm{E}_{s} f(k)=\left[\frac{Q}{2}+i s-i k\right]_{b} f(k+i b), \quad \\
& \mathrm{F}_{s} f(k)=\left[\frac{Q}{2}+i s+i k\right]_{b} f(k-i b),
\end{aligned}
$$

where $[x]_{b} \equiv \frac{\sin \pi b x}{\sin \pi b^{2}}$.

The distinguished role of the space $\mathcal{T}_{s}$ is explained by the following result, which shows that the space $\mathcal{T}_{s}$ is canonically associated to the representation $\pi_{s}$ :

Lemma 4. $\mathcal{T}_{s}$ is the largest space on which all $\pi_{s}(u), u \in \mathcal{U}_{q}(\mathfrak{s l}(2, \mathbb{R}))$ are well-defined, i.e.,

$$
\mathcal{T}_{s}=\bigcap_{u \in \mathcal{B}_{q}(\mathfrak{s}((2, \mathbb{R}))} \mathcal{D}_{u}
$$

where $\mathcal{D}_{u}$ is the domain of the unbounded operator $\pi_{s}(u), u \in \mathcal{B}_{q}(\mathfrak{s l}(2, \mathbb{R}))$. The spaces $\mathcal{T}_{s}, s \in \mathbb{R}$ are Fréchet spaces with topology defined by the family of seminorms

$$
\|f\|_{u} \equiv \sup _{k \in \mathbb{R}}\left|\left(\pi_{s}(u) f\right)(k)\right|, \quad u \in \mathcal{B}_{q}(\mathfrak{s l}(2, \mathbb{R})) .
$$

Proof. It is easy to check that $\pi_{s}(u) f \in \mathcal{T}_{s}$ for all $f \in \mathcal{T}_{s}$. In order to show that the conditions in the definition of $\mathcal{T}_{s}$ are all necessary, let us first observe that $e^{a|k|} f \in L^{2}(\mathbb{R})$ is clearly necessary for $\mathrm{K}_{s}^{n} f$ to be well-defined for all $n \in \mathbb{Z}$. In order to determine the conditions on $f$ for $\mathrm{E}_{s}^{n} f$ to be well-defined, let us consider the unitary operator $\mathrm{U}_{s} \equiv w_{b}(\mathrm{p}-s)$, where the special function $w_{b}(x)$ and its properties are described in Appendix A.2. We then have

$$
\mathrm{U}_{s} \cdot \mathrm{E}_{s}^{n} \cdot \mathbf{U}_{s}^{-1}=e^{2 \pi n b \mathrm{x}} .
$$


The intersection of the domains of $e^{2 \pi n b x}$ for all $n \in \mathbb{N}$ consists of functions $g(k)$ that are analytic in the upper half plane $\mathbb{H}_{+}$, see, e.g., [S, Lemma 1]. The corresponding functions $f(k)=\left(\mathrm{U}_{s}^{-1} g\right)(k)=\left(w_{b}(k-s)\right)^{-1} g(k)$ may have poles in $\Upsilon_{s}$. Similar arguments applied to $\mathrm{F}_{s}^{n}$ allow us to complete the proof of the first statement in Lemma4

In order to verify the second statement, we mainly have to show that the space $\mathcal{T}_{s}$ is complete w.r.t. the topology defined by the seminorms (B.4). This follows from (B.3) together with the observation that the self-adjoint operators $\pi_{s}(u), u \in \mathcal{B}_{q}(\mathfrak{s l}(2, \mathbb{R}))$ are closed on $\mathcal{D}_{u}$.

We regard Lemma 4 as the key to the mathematical understanding of the duality $b \rightarrow b^{-1}$ of our representations $\mathcal{P}_{s}$. Indeed, let us introduce the operators $\tilde{\mathrm{E}}_{s}, \tilde{\mathrm{F}}_{s}, \tilde{\mathrm{K}}_{s}$, obtained by replacing $b \rightarrow b^{-1}$ in [6. These operators generate a representation $\tilde{\mathcal{P}}_{s}$ of $\mathcal{U}_{\tilde{q}}(\mathfrak{s l}(2, \mathbb{R})), \tilde{q}=e^{i \pi b^{-2}}$ on the same space $\mathcal{T}_{s}$. The space $\mathcal{T}_{s}$ is associated to the representation $\tilde{\mathcal{P}}_{s}$ as canonically as it is associated to $\mathcal{P}_{s}$. Moreover, it is easy to see that the representation $\tilde{\mathcal{P}}_{s}$ commutes ${ }^{6}$ with $\mathcal{P}_{s}$ on $\mathcal{T}_{s}$. It is therefore natural to regard $\mathcal{T}_{s}$ as the natural space on which a representation of the modular double $\mathcal{U}_{q}(\mathfrak{s l}(2, \mathbb{R})) \otimes \mathcal{U}_{\tilde{q}}(\mathfrak{s l}(2, \mathbb{R}))[\overline{\mathrm{F}} 3]$ is realized.

Another way to make the self-duality of the representations $\mathcal{P}_{s}$ transparent uses the rescaled generators introduced in (11). These generators and their counterparts $\tilde{\mathrm{e}}_{s}, \tilde{\mathrm{f}}_{s}, \tilde{\mathrm{k}}_{s}$, obtained by replacing $b$ with $b^{-1}$ are related as [BT]

$$
\left(\mathrm{e}_{s}\right)^{\frac{1}{b}}=\left(\tilde{\mathrm{e}}_{s}\right)^{b}, \quad\left(\mathrm{f}_{s}\right)^{\frac{1}{b}}=\left(\tilde{\mathrm{f}}_{s}\right)^{b}, \quad\left(\mathrm{k}_{s}\right)^{\frac{1}{b}}=\left(\tilde{\mathrm{k}}_{s}\right)^{b}
$$

These observations express quite clearly that the representations of the two halves of the modular double, $\mathcal{U}_{q}(\mathfrak{s l}(2, \mathbb{R}))$ and $\mathcal{U}_{\tilde{q}}(\mathfrak{s} l(2, \mathbb{R}))$, are related to each other like the two sides of the same coin.

\section{Structure of the monodromy matrix}

This appendix is devoted to the derivation of some simple, but important structural properties of the monodromy matrix $\mathrm{M}(u)$,

$$
\mathrm{M}(u) \equiv\left(\begin{array}{ll}
\mathrm{A}_{\mathrm{N}}(u) & \mathrm{B}_{\mathrm{N}}(u) \\
\mathrm{C}_{\mathrm{N}}(u) & \mathrm{D}_{\mathrm{N}}(u)
\end{array}\right) \equiv L_{\mathrm{N}}(u) \cdot \ldots \cdot L_{2}(u) \cdot L_{1}(u)
$$

\section{C.1 Expansions in the spectral parameter}

Introduce the following notations for $n \in \mathbb{N}$,

$$
\lceil n\rceil \equiv\left\{\begin{array}{ll}
n-1 & \text { if } n \text {-odd; } \\
n & \text { if } n \text {-even; }
\end{array}, \quad\lfloor n\rfloor \equiv \begin{cases}n & \text { if } n \text {-odd; } \\
n-1 & \text { if } n \text {-even; }\end{cases}\right.
$$

\footnotetext{
${ }^{6}$ Commutativity of $\mathcal{P}_{s}$ and $\tilde{\mathcal{P}}_{s}$ only holds on the dense domain $\mathcal{T}_{s}$ but not in the usual sense of commutativity of spectral projections!
} 
Lemma 5. The elements $\mathrm{A}_{\mathrm{N}}(u), \mathrm{B}_{\mathrm{N}}(u)$, and $\mathrm{D}_{\mathrm{N}}(u)$ of $\mathrm{M}(u)$ have the following form:

$$
\begin{gathered}
\mathrm{A}_{\mathrm{N}}^{\mathrm{Xxz}}(u)=e^{\mathrm{N} \pi b u} \sum_{m=0}^{\mathrm{N}}(-)^{m} e^{-2 m \pi b u} \mathrm{~A}_{\mathrm{N}, m}^{\mathrm{xxz}}, \\
\mathrm{B}_{\mathrm{N}}^{\mathrm{Xxz}}(u)=i e^{\mathrm{N} \pi b u} \sum_{m=0}^{\mathrm{N}-1}(-)^{m} e^{-2 m \pi b u} \mathrm{~B}_{\mathrm{N}, m}^{\mathrm{XXz}}, \\
\mathrm{D}_{\mathrm{N}}^{\mathrm{Xxz}}(u)=e^{\mathrm{N} \pi b u} \sum_{m=0}^{\mathrm{N}}(-)^{m} e^{-2 m \pi b u} \mathrm{D}_{\mathrm{N}, m}^{\mathrm{Xxz}}, \\
\mathrm{A}_{\mathrm{N}}^{\mathrm{SG}}(u)=i^{\lceil\mathrm{N}\rceil} e^{\pi b(\lceil\mathrm{~N}\rceil u-\mathrm{N} s)} \sum_{m=0}^{\lceil\mathrm{N}\rceil}(-)^{m} e^{-2 m \pi b u} \mathrm{~A}_{\mathrm{N}, m}^{\mathrm{SG}}, \\
\mathrm{B}_{\mathrm{N}}^{\mathrm{SG}}(u)=-i^{\lfloor\mathrm{N}\rfloor} e^{\pi b(\lfloor\mathrm{~N}\rfloor u-\mathrm{N} s)} \sum_{m=0}^{\lfloor\mathrm{N}\rfloor}(-)^{m} e^{-2 m \pi b u} \mathrm{~B}_{\mathrm{N}, m}^{\mathrm{SG}}, \\
\mathrm{D}_{\mathrm{N}}^{\mathrm{SG}}(u)=i^{\lceil\mathrm{N}\rceil} e^{\pi b(\lceil\mathrm{~N}\rceil u-\mathrm{N} s)} \sum_{m=0}^{\lceil\mathrm{N}\rceil}(-)^{m} e^{-2 m \pi b u} \mathrm{D}_{\mathrm{N}, m}^{\mathrm{SG}},
\end{gathered}
$$

where $\mathrm{A}_{\mathrm{N}, m}, \mathrm{~B}_{\mathrm{N}, m}$, and $\mathrm{D}_{\mathrm{N}, m}$ are positive self-adjoint operators.

Proof. Let us consider the case of the XXZ chain, the other case being very similar. The definition of the monodromy matrix $\mathrm{M}_{\mathrm{N}}(u)$ yields the following recursion relations

$$
\begin{aligned}
& \mathrm{A}_{\mathrm{N}}^{\mathrm{xxz}}(u)=\left(e^{\pi b u} \mathrm{k}_{\mathrm{N}}-e^{-\pi b u} \mathrm{k}_{\mathrm{N}}^{-1}\right) \mathrm{A}_{\mathrm{N}-1}^{\mathrm{xxz}}(u)+i e^{\pi b u}{ }_{f_{\mathrm{N}}} \mathrm{C}_{\mathrm{N}-1}^{\mathrm{xxz}}(u), \\
& \mathrm{B}_{\mathrm{N}}^{\mathrm{xxz}}(u)=\left(e^{\pi b u} \mathrm{k}_{\mathrm{N}}-e^{-\pi b u} \mathrm{k}_{\mathrm{N}}^{-1}\right) \mathrm{B}_{\mathrm{N}-1}^{\mathrm{xxz}}(u)+i e^{\pi b u} \mathrm{f}_{\mathrm{N}} \mathrm{D}_{\mathrm{N}-1}^{\mathrm{xxz}}(u), \\
& \mathrm{C}_{\mathrm{N}}^{\mathrm{xxz}}(u)=\left(e^{\pi b u} \mathrm{k}_{\mathrm{N}}^{-1}-e^{-\pi b u} \mathrm{k}_{\mathrm{N}}\right) \mathrm{C}_{\mathrm{N}-1}^{\mathrm{xxz}}(u)+i e^{-\pi b u} e_{\mathrm{N}} \mathrm{A}_{\mathrm{N}-1}^{\mathrm{xxz}}(u), \\
& \mathrm{D}_{\mathrm{N}}^{\mathrm{xxz}}(u)=\left(e^{\pi b u} \mathrm{k}_{\mathrm{N}}^{-1}-e^{-\pi b u} \mathrm{k}_{\mathrm{N}}\right) \mathrm{D}_{\mathrm{N}-1}^{\mathrm{xxz}}(u)+i e^{-\pi b u} \mathrm{e}_{\mathrm{N}} \mathrm{B}_{\mathrm{N}-1}^{\mathrm{xx}}(u),
\end{aligned}
$$

where $k_{N} \equiv k_{s} \otimes 1 \otimes \ldots 1$ etc. Using these recursion relations one may inductively show that the operators $\mathrm{A}_{\mathrm{N}, m}, \mathrm{~B}_{\mathrm{N}, m}$ and $\mathrm{D}_{\mathrm{N}, m}$ are linear combinations of monomials of the form

$$
\mathrm{u}_{s}^{(\mathrm{N})} \otimes \ldots \otimes \mathrm{u}_{s}^{(1)}, \quad \mathrm{u}_{s}^{k} \in\left\{\mathrm{e}_{s}, \mathrm{f}_{s}, \mathrm{k}_{s}, \mathrm{k}_{s}^{-1}\right\}
$$

with positive integer coefficients. It remains to note that an operator which is the sum of positive self-adjoint operators will be self-adjoint on the intersection of the domains of the individual summands. These obserations reduce our claim to the self-adjointness and positivity of $\mathrm{e}_{s}, \mathrm{f}_{s}, \mathrm{k}_{s}$.

Lemma 6. The leading terms of $\mathrm{A}_{\mathrm{N}}(u), \mathrm{B}_{\mathrm{N}}(u)$, and $\mathrm{D}_{\mathrm{N}}(u)$ at $e^{\pi b u} \rightarrow \pm \infty$ are given by

$$
\begin{array}{ll}
\mathrm{B}_{\mathrm{N}, \mathrm{o}}^{\mathrm{XXZ}}=\Delta^{(\mathrm{N}-1)} \mathrm{f}, & \mathrm{A}_{\mathrm{N}, \mathrm{o}}^{\mathrm{XXZ}}=\mathrm{D}_{\mathrm{N}, \mathrm{N}}^{\mathrm{XxZ}}=\Delta^{(\mathrm{N}-1)} \mathrm{k}, \\
\mathrm{B}_{\mathrm{N}, \mathrm{N}-1}^{\mathrm{XXZ}}=\bar{\Delta}^{(\mathrm{N}-1)} \mathrm{f}, & \mathrm{A}_{\mathrm{N}, \mathrm{N}}^{\mathrm{XXZ}}=\mathrm{D}_{\mathrm{N}, \mathrm{o}}^{\mathrm{XXZ}}=\Delta^{(\mathrm{N}-1)} \mathrm{k}^{-1},
\end{array}
$$


where $\Delta^{(n)}$ is the $n$-fold co-product defined via $\Delta^{(n+1)}=\left(\Delta^{(n)} \otimes i d\right) \circ \Delta$, with $\Delta^{(0)} \equiv i d$ and $\Delta^{(1)} \equiv \Delta$, and $\bar{\Delta}^{(n)}$ is defined analogously for the opposite co-product $\bar{\Delta}^{(1)} \equiv \Delta^{\prime}$.

$$
\begin{aligned}
\mathrm{N}-\text { odd }: & \begin{cases}\mathrm{B}_{\mathrm{N}, \mathrm{o}}^{\mathrm{SG}}=\theta_{\text {odd }}\left(\Delta^{(\mathrm{N}-1)} \mathrm{k}^{-1}\right), & \mathrm{B}_{\mathrm{N}, \mathrm{N}}^{\mathrm{SG}}=\theta_{\text {odd }}\left(\Delta^{(\mathrm{N}-1)} \mathrm{k}\right), \\
\mathrm{A}_{\mathrm{N}, \mathrm{o}}^{\mathrm{SG}}=\theta_{\text {odd }}\left(\Delta^{(\mathrm{N}-1)} \mathrm{f}\right), & \mathrm{A}_{\mathrm{N}, \mathrm{N}-1}^{\mathrm{SG}}=\theta_{\text {odd }}\left(\bar{\Delta}^{(\mathrm{N}-1)} \mathrm{f}\right), \\
\mathrm{D}_{\mathrm{N}, \mathrm{o}}^{\mathrm{SG}}=\theta_{\text {odd }}\left(\bar{\Delta}^{(\mathrm{N}-1)} \mathrm{e}\right), & \mathrm{D}_{\mathrm{N}, \mathrm{N}-1}^{\mathrm{SG}}=\theta_{\text {odd }}\left(\Delta^{(\mathrm{N}-1)} \mathrm{e}\right),\end{cases} \\
\mathrm{N} \text { - even: } \quad & \begin{cases}\mathrm{B}_{\mathrm{N}, \mathrm{o}}^{\mathrm{SG}}=\theta_{\text {even }}\left(\Delta^{(\mathrm{N}-1)} \mathrm{f}\right), & \mathrm{B}_{\mathrm{N}, \mathrm{N}-1}^{\mathrm{SG}}=\theta_{\text {even }}\left(\bar{\Delta}^{(\mathrm{N}-1)} \mathrm{f}\right), \\
\mathrm{A}_{\mathrm{N}, \mathrm{o}}^{\mathrm{SG}}=\theta_{\text {even }}\left(\Delta^{(\mathrm{N}-1)} \mathrm{k}\right), & \mathrm{A}_{\mathrm{N}, \mathrm{N}}^{\mathrm{SG}}=\theta_{\text {even }}\left(\Delta^{(\mathrm{N}-1)} \mathrm{k}^{-1}\right), \\
\mathrm{D}_{\mathrm{N}, \mathrm{o}}^{\mathrm{SG}}=\theta_{\text {even }}\left(\Delta^{(\mathrm{N}-1)} \mathrm{k}^{-1}\right), & \mathrm{D}_{\mathrm{N}, \mathrm{N}}^{\mathrm{SG}}=\theta_{\text {even }}\left(\Delta^{(\mathrm{N}-1)} \mathrm{k}\right),\end{cases}
\end{aligned}
$$

where $\theta_{\text {odd }} \equiv \theta_{\mathrm{N}-1} \circ \ldots \theta_{3} \circ \theta_{1}$ and $\theta_{\text {even }} \equiv \theta_{\mathrm{N}} \circ \ldots \theta_{4} \circ \theta_{2}$ are compositions of the automorphism (37) at oddleven sites.

Proof. Eqs. C.13 follow easily from the decomposition (14) of the corresponding L-matrix. Eqs. C.15) are obtained by analogous consideration if $L^{\mathrm{SG}}(u)$ is replaced with $\sigma_{1} L^{\prime}(u)$ (see (41)) and formula (42) is used. In order to apply this approach in the $\mathrm{N}$ odd case, one has to multiply the monodromy matrix with an extra $\sigma_{1}$ from the right (which leads to the interchange $\mathrm{A} \leftrightarrow \mathrm{B}$ and $\mathrm{C} \leftrightarrow \mathrm{D})$.

\section{C.2 Quantum determinant}

Let us discuss connection between the so called quantum determinant and coefficients $a(u)$ and $d(u)$ which arise in the Baxter equation (62). Since for the modular magnet we use R-matrix (15) which is not symmetric, the corresponding quantum determinant will differ from the "standard" formula applicable, e.g., for the sinh-Gordon model. Therefore we commence by deriving the required expression.

Lemma 7. Let $L(u)$ be an $L-$ matrix, satisfying the relation (12) with the auxiliary $R$-matrix of the form

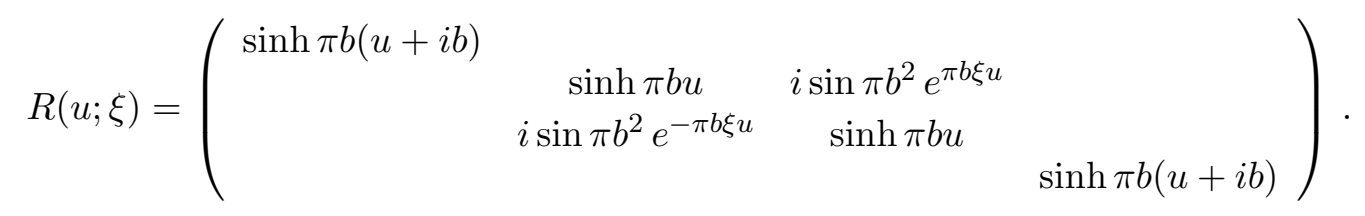

and let $\mathrm{M}(u)$ be the corresponding monodromy matrix defined by (C.1). The following element (quantum determinant)

$$
\operatorname{det}_{q} \mathrm{M}(u)=\mathrm{A}(u) \mathrm{D}(u-i b)-q^{-\xi} \mathrm{B}(u) \mathrm{C}(u-i b)
$$

is central, i.e., $\left[\mathrm{M}(v), \operatorname{det}_{q} \mathrm{M}(u)\right]=0$, and can be written as follows

$$
\operatorname{det}_{q} \mathrm{M}(u)=\left(\operatorname{det}_{q} L(u)\right)^{\mathrm{N}},
$$

where the quantum determinant of $L(u)$ is defined by the same formula (C.17) (with $\mathrm{M}(u)$ replaced by $L(u))$. 
Proof. Existence of the quantum determinant is due to the degeneration of the auxiliary R-matrix $\check{R}(u ; \xi) \equiv \mathrm{P} R(u ; \xi)$ at $u=-i b$,

$$
\check{R}(-i b ; \xi)=i \sin \pi b^{2}\left(\begin{array}{cccc}
0 & & & \\
& q^{\xi} & -1 & \\
& -1 & q^{-\xi} & \\
& & & 0
\end{array}\right) .
$$

It is interesting to notice that this matrix is proportional (in the standard basis) to the onedimensional projector $P_{\xi}^{-}$onto the spin 0 representation in the tensor square of spin $\frac{1}{2}$ representations of $\mathcal{U}_{q^{\xi}}(\mathfrak{s u}(2))$.

For $R(u ; 0)$ the statement of the Lemma is well-known (see, e.g., [KBI]). In the generic case $\xi \neq 0$, one can observe that the gauge transformation

$$
\tilde{L}(u)=g_{u}^{-1} L(u) g_{u}, \quad \tilde{\mathrm{M}}(u)=g_{u}^{-1} \mathrm{M}(u) g_{u}, \quad g_{u}=e^{\frac{1}{2} \pi b \xi u \sigma_{3}}
$$

yields L-matrix and monodromy matrix which satisfy the relation (12) with the auxiliary R-matrix $R(u ; 0)$. Therefore

$$
\operatorname{det}_{q} \tilde{\mathrm{M}}(u)=\tilde{\mathrm{A}}(u) \tilde{\mathrm{D}}(u-i b)-\tilde{\mathrm{B}}(u) \tilde{\mathrm{C}}(u-i b)
$$

commutes with entries of $\tilde{M}(u)$ and hence with entries of $\mathrm{M}(u)$. Using (C.20) in order to rewrite (C.21) in terms of entries of $\mathrm{M}(u)$, we obtain (C.17).

In order to prove (C.18) it suffices to observe that

$$
\operatorname{det}_{q} \mathrm{M}(u) \cdot \check{R}_{12}(-i b ; \xi)=\mathrm{M}_{1}(u) \mathrm{M}_{2}(u-i b) \check{R}_{12}(-i b ; \xi),
$$

which yields also three different expressions equivalent to (C.17) if we take into account the relation $\check{R}_{12}(-i b ; \xi) \mathrm{M}_{1}(u-i b) \mathrm{M}_{2}(u)=\mathrm{M}_{1}(u) \mathrm{M}_{2}(u-i b) \check{R}_{12}(-i b ; \xi)$ which is a particular case of (12). Now if $\mathrm{M}^{\prime}(u), \mathrm{M}^{\prime \prime}(u)$ satisfy (12) with the same R-matrix and their entries commute, then we have

$$
\begin{aligned}
& \operatorname{det}_{q}\left(\mathrm{M}^{\prime}(u) \mathrm{M}^{\prime \prime}(u)\right) \cdot \check{R}_{12}(-i b ; \xi)=\mathrm{M}_{1}^{\prime}(u) \mathrm{M}_{1}^{\prime \prime}(u) \mathrm{M}_{2}^{\prime}(u-i b) \mathrm{M}_{2}^{\prime \prime}(u-i b) \check{R}_{12}(-i b ; \xi) \\
& =\mathrm{M}_{1}^{\prime}(u) \mathrm{M}_{2}^{\prime}(u-i b) \mathrm{M}_{1}^{\prime \prime}(u) \mathrm{M}_{2}^{\prime \prime}(u-i b) \check{R}_{12}(-i b ; \xi)=\operatorname{det}_{q} \mathrm{M}^{\prime}(u) \cdot \operatorname{det}_{q} \mathrm{M}^{\prime \prime}(u) .
\end{aligned}
$$

Whence (C.18 follows immediately.

For the models that we consider, Lemma 7 yields

$$
\begin{aligned}
\operatorname{det}_{q} \mathrm{M}^{\mathrm{xxz}}(u) & =\mathrm{A}(u) \mathrm{D}(u-i b)-q^{-1} \mathrm{~B}(u) \mathrm{C}(u-i b) \\
& =\left(4 \cosh \pi b\left(s+u-i \frac{b}{2}\right) \cosh \pi b\left(s-u+i \frac{b}{2}\right)\right)^{\mathrm{N}}, \\
\operatorname{det}_{q} \mathrm{M}^{\mathrm{SG}}(u) & =\mathrm{A}(u) \mathrm{D}(u-i b)-\mathrm{B}(u) \mathrm{C}(u-i b) \\
& =\left(4 e^{-2 \pi b s} \cosh \pi b\left(s+u-i \frac{b}{2}\right) \cosh \pi b\left(s-u+i \frac{b}{2}\right)\right)^{\mathrm{N}} .
\end{aligned}
$$

Proving the Proposition 5 in Appendix E, we will deal with monodromy matrices $\tilde{\mathrm{M}}(u)$ obtained from $\mathrm{M}(u)$ by a gauge transformation with a matrix $G(u)$. Notice that such $\tilde{\mathrm{M}}(u)$ satisfies the exchange relation (12) with an $\mathrm{R}$-matrix

$$
\tilde{R}_{12}(u, v ; \xi)=G_{1}(u) G_{2}(v) R(u-v ; \xi) G_{1}^{-1}(u) G_{2}^{-1}(v)
$$


which is not of the form (C.16). Therefore, for general $G(u)$, Lemma 7 does not apply to $\tilde{\mathrm{M}}(u)$. Nevertheless, there exists a class of gauge transformations which preserve the quantum determinant in the following sense.

Lemma 8. Let $\mathrm{M}(u)$ satisfy (12) with the auxiliary $R$-matrix $R(u ; \xi)$ of the form (C.16). Let $\tilde{\mathrm{M}}(u)=\left(\begin{array}{cc}\tilde{A}(u) & \tilde{B}(u) \\ \tilde{C}(u) & \tilde{D}(u)\end{array}\right)$ be its gauge transform defined by

$$
\tilde{\mathrm{M}}(u)=G(u) \cdot \mathrm{M}(u) \cdot G^{-1}(u), \quad G(u)=\left(\begin{array}{cc}
1 & 0 \\
\rho & 1
\end{array}\right), \quad \rho=e^{-\pi b \xi u} \rho_{0},
$$

where $\rho_{0}$ is a c-number, which does not depend on $u$. Then

$$
\operatorname{det}_{q} \tilde{\mathrm{M}}(u) \equiv \tilde{\mathrm{A}}(u) \tilde{\mathrm{D}}(u-i b)-q^{-\xi} \tilde{\mathrm{B}}(u) \tilde{\mathrm{C}}(u-i b)=\operatorname{det}_{q} \mathrm{M}(u),
$$

where the r.h.s. is defined according to Lemma 7

Since entries of $\tilde{M}(u)$ are linear combinations of entries of $M(u)$, it follows that $\operatorname{det}_{q} \tilde{M}(u)$ is central, i.e., $\left[\tilde{\mathrm{M}}(v), \operatorname{det}_{q} \tilde{\mathrm{M}}(u)\right]=0$. Thus, the 1.h.s. defines the quantum determinant corresponding to the R-matrix (C.28.

Proof. Although $R(u-v ; \xi)$ and $\tilde{R}(u, v ; \xi)$ are in general not equal, they coincide at $u-v=-i b$. Indeed, using the explicit form of $G(u)$, it is easy to check that

$$
G_{1}(u) G_{2}(u-i b) \check{R}(-i b ; \xi)=\check{R}(-i b ; \xi) .
$$

Observing also that (C.22) holds for $\operatorname{det}_{q} \tilde{\mathrm{M}}(u)$ as well, we derive

$$
\begin{aligned}
& \operatorname{det}_{q} \tilde{\mathrm{M}}(u) \cdot \check{R}_{12}(-i b ; \xi)=\tilde{\mathrm{M}}_{1}(u) \tilde{\mathrm{M}}_{2}(u-i b) \check{R}_{12}(-i b ; \xi) \\
& =G_{1}(u) \mathrm{M}_{1}(u) G_{1}^{-1}(u) G_{2}(u-i b) \mathrm{M}_{2}(u-i b) G_{2}^{-1}(u-i b) \check{R}_{12}(-i b ; \xi) \\
& =G_{1}(u) G_{2}(u-i b) \mathrm{M}_{1}(u) \mathrm{M}_{2}(u-i b) G_{2}^{-1}(u-i b) G_{1}^{-1}(u) \check{R}_{12}(-i b ; \xi) \\
& =G_{1}(u) G_{2}(u-i b) \mathrm{M}_{1}(u) \mathrm{M}_{2}(u-i b) \check{R}_{12}(-i b ; \xi) \\
& =G_{1}(u) G_{2}(u-i b) \check{R}_{12}(-i b ; \xi) \cdot \operatorname{det}_{q} \mathrm{M}(u)=\check{R}_{12}(-i b ; \xi) \cdot \operatorname{det}_{q} \mathrm{M}(u),
\end{aligned}
$$

which proves the assertion of the Lemma.

It is important that the gauge transformations used in the proof of the Proposition 5 belong to the class of gauge transformations described in Lemma 8 , they correspond to $\xi=1$ and $\xi=0$, respectively. This fact allows us to relate the quantum determinants of the models in question and the coefficients of the corresponding Baxter equations. A quick inspection of the proof of Proposition 5 shows that

$$
(a(u) d(u-i b))^{\mathrm{N}}=\operatorname{det}_{q} \mathrm{M}(u),
$$

where the r.h.s. is given by (C.25 and (C.27), respectively.

\section{Construction of the fundamental $\mathbf{R}$-operator $\mathrm{R}(u)$}

For the proof of Theorem 1 we will need the following material from [PT2 [BT]. 


\section{D.1 Clebsch-Gordan maps}

Let the space $\mathcal{M}$ be defined by the direct integral

$$
\mathcal{M} \equiv \int_{\mathbb{R}^{+}}^{\oplus} d s \mathcal{P}_{s}
$$

Realizing elements of $\mathcal{T}_{s}$ as functions $f(k)$ leads us to represent the elements of $\mathcal{M}$ by families of functions $f \equiv\left(f_{s} ; s \in \mathbb{R}^{+}\right)$, where $f_{s} \equiv f_{s}(k) \in \mathcal{T}_{s}$ for all $s \in \mathbb{R}^{+}$. We shall define the multiplication operator s by

$$
\mathrm{s} f=\left(s f_{s} ; s \in \mathbb{R}^{+}\right) .
$$

To any family $\left(\mathrm{O}_{s} ; s \in \mathbb{R}^{+}\right)$of operators on $\mathcal{T}_{s}$ we may then associate an operator $\mathrm{O}_{\mathrm{s}}$ on $\mathcal{M}$ in the obvious manner. We have the corresponding canonical action of $\mathcal{U}_{q}(\mathfrak{s l}(2, \mathbb{R}))$ on $\mathcal{M}$ via

$$
\hat{\pi}_{\mathbf{s}}(X) f=\left(\pi_{s}(X) f_{s} ; s \in \mathbb{R}^{+}\right), \quad \forall X \in \mathcal{U}_{q}(\mathfrak{s l}(2, \mathbb{R}))
$$

The Clebsch-Gordan maps $\mathrm{C}_{s_{2} s_{1}}$ were defined in [PT1, [PT2] as a family of operators

$$
\mathrm{C}_{s_{2} s_{1}}: \mathcal{P}_{s_{2}} \otimes \mathcal{P}_{s_{1}} \longrightarrow \mathcal{M}
$$

The Clebsch-Gordan maps $C_{s_{2} s_{1}}$ intertwine the action of $\mathcal{U}_{q}(\mathfrak{s l}(2, \mathbb{R}))$ on $\mathcal{P}_{s_{2}} \otimes \mathcal{P}_{s_{1}}$ with the canonical action on $\mathcal{M}$ in the sense that

$$
\mathrm{C}_{s_{2} s_{1}} \cdot\left(\pi_{s_{2}} \otimes \pi_{s_{1}}\right) \Delta(X)=\hat{\pi}_{\mathbf{s}}(X) \cdot \mathrm{C}_{s_{2} s_{1}} \quad \forall X \in \mathcal{U}_{q}(\mathfrak{s l}(2, \mathbb{R}))
$$

\section{D.2 R-operator and braiding}

Let us introduce

$$
\mathrm{R}_{s_{2} s_{1}}=q^{\mathrm{H}_{s_{2}} \otimes \mathrm{H}_{s_{1}}} g_{b}\left(\mathrm{e}_{s_{2}} \otimes \mathrm{f}_{s_{1}}\right) q^{\mathrm{H}_{s_{2}} \otimes \mathrm{H}_{s_{1}}} .
$$

Here the anti-self-adjoint operator $\mathrm{H}_{s}$ is defined by $\mathrm{k}_{s}=q^{\mathrm{H}_{s}}$, and $g_{b}(x)$ is the non-compact quantum dilogarithm related to the special function $w_{b}(x)$, defined in Appendix $\mathrm{A}$ via

$$
g_{b}\left(e^{2 \pi b x}\right)=e^{\frac{\pi i}{24}\left(b^{2}+b^{-2}\right)+\frac{\pi i}{2} x^{2}} w_{b}(-x) .
$$

The $\mathrm{R}$-operator $\mathrm{R}_{s_{2} s_{1}}$ satisfies the following relations $[\overline{\mathrm{BT}}]$

$$
\begin{aligned}
& \mathrm{R}_{s_{2} s_{1}} \cdot\left(\pi_{s_{2}} \otimes \pi_{s_{1}}\right) \Delta(X)=\left(\pi_{s_{2}} \otimes \pi_{s_{1}}\right) \Delta^{\prime}(X) \cdot \mathrm{R}_{s_{2} s_{1}}, \\
& \mathrm{R}_{s_{2} s_{1}}\left(\mathrm{e}_{s_{2}} \otimes \mathrm{k}_{s_{1}}^{-1}\right)=\left(\mathrm{e}_{s_{2}} \otimes \mathrm{k}_{s_{1}}\right) \mathrm{R}_{s_{2} s_{1}}, \\
& \mathrm{R}_{s_{2} s_{1}}\left(\mathrm{k}_{s_{2}} \otimes \mathrm{f}_{s_{1}}\right)=\left(\mathrm{k}_{s_{2}}^{-1} \otimes \mathrm{f}_{s_{1}}\right) \mathrm{R}_{s_{2} s_{1}},
\end{aligned}
$$

where $X \in \mathcal{U}_{q}(\mathfrak{s l}(2, \mathbb{R}))$ and $\Delta^{\prime}$ stands for the opposite co-product.

The braiding operator $\mathrm{B}: \mathcal{P}_{s_{2}} \otimes \mathcal{P}_{s_{1}} \rightarrow \mathcal{P}_{s_{1}} \otimes \mathcal{P}_{s_{2}}$ is defined by $\mathrm{B}_{s_{2} s_{1}}=\mathrm{PR}_{s_{2} s_{1}}$, where $\mathrm{P}$ is the operator that permutes the two tensor factors. In what follows we will need the following statement. 
Proposition 3 (Theorem 6 in [BT]). The braiding operator is diagonalized by the ClebschGordan maps in the following sense:

$$
\mathrm{C}_{s_{1} s_{2}} \cdot \mathrm{B}_{s_{2} s_{1}}=\Omega_{s_{2} s_{1}}^{\mathrm{s}} \cdot \mathrm{C}_{s_{2} s_{1}}
$$

where $\Omega_{s_{2} s_{1}}^{\mathrm{s}}$ is the operator on $\mathcal{M}$ associated via (D.2) to the scalar function

$$
\Omega_{s_{2} s_{1}}^{s}=e^{\pi i\left(s_{1}^{2}+s_{2}^{2}-s^{2}+\frac{Q^{2}}{4}\right)} .
$$

In the particular case, $s_{2}=s_{1}$, one can regard the permutation $\mathrm{P}$ as an endomorphism of $\mathcal{P}_{s_{1}} \otimes$ $\mathcal{P}_{s_{1}}$ and then Proposition 3 allows to relate it to the R-operator:

$$
\mathrm{P}=\mathrm{R}_{s_{1} s_{1}} e^{-\pi i\left(\frac{Q^{2}}{4}+2 s_{1}^{2}-\mathrm{s}^{2}\right)} .
$$

\section{D.3 Proof of Theorem 1}

We will consider the more general R-operator defined on $\mathcal{P}_{s_{2}} \otimes \mathcal{P}_{s_{1}}$ by the formula

$$
\mathrm{R}_{s_{2} s_{1}}(u)=\mathrm{R}_{s_{2} s_{1}} e^{+\pi i\left(\mathrm{~s}_{21}^{2}-s_{1}^{2}-s_{2}^{2}-\frac{Q^{2}}{4}\right)} D_{u}\left(\mathrm{~s}_{21}\right),
$$

where the special function $D_{\alpha}(x)$ is defined by Eq. A.19 in Appendix $\mathrm{A}$ and $\mathrm{s}_{21}$ is the unique positive self-adjoint operator such that

$$
4 \cosh ^{2} \pi b s_{21}=\left(\pi_{s_{2}} \otimes \pi_{s_{1}}\right) \Delta(C) .
$$

It follows easily from relation (D.13) that $\mathrm{R}(u) \equiv \mathrm{R}_{s s}(u)$ coincides with the fundamental Roperator defined earlier in (20):

$$
\mathrm{R}(u)=\mathrm{P} w_{b}(u+\mathrm{s}) w_{b}(u-\mathrm{s})=\mathrm{P} D_{u}(\mathrm{~s}) .
$$

To begin the proof of Theorem 11 which is somewhat more involved than the proofs of the analogous results in the case of highest weight representations [Ji] [F1, BD], let us observe that Eq. (19) is equivalent to the following set of equations

$$
\begin{aligned}
& \mathrm{R}_{s_{2} s_{1}}(u) \Delta\left(L^{ \pm}\right)=\Delta^{\prime}\left(L^{ \pm}\right) \mathrm{R}_{s_{2} s_{1}}(u), \\
& \mathrm{R}_{s_{2} s_{1}}(u) \ell_{i j}(u)=\tau\left(\ell_{i j}(-u)\right) \mathrm{R}_{s_{2} s_{1}}(u), \quad i, j=1,2,
\end{aligned}
$$

where $L^{ \pm}$were introduced in (13)-14], $\tau$ is the flip operation: $\tau\left(a_{s_{2}} \otimes b_{s_{1}}\right)=b_{s_{2}} \otimes a_{s_{1}}$, and the operators $\ell_{i j}(u)$ are given by

$$
\begin{aligned}
& \ell_{11}(u)=e^{\pi b u} \mathrm{e}_{s_{2}} \otimes \mathrm{f}_{s_{1}}+e^{\pi b u} \mathrm{k}_{s_{2}}^{-1} \otimes \mathrm{k}_{s_{1}}+e^{-\pi b u} \mathrm{k}_{s_{2}} \otimes \mathrm{k}_{s_{1}}^{-1}, \\
& \ell_{12}(u)=e^{\pi b u} \mathrm{k}_{s_{2}} \otimes \mathrm{f}_{s_{1}}+e^{-\pi b u} \mathrm{f}_{s_{2}} \otimes \mathrm{k}_{s_{1}}^{-1}, \\
& \ell_{21}(u)=e^{\pi b u} \mathrm{e}_{s_{2}} \otimes \mathrm{k}_{s_{1}}^{-1}+e^{-\pi b u} \mathrm{k}_{s_{2}} \otimes \mathrm{e}_{s_{1}}, \\
& \ell_{22}(u)=e^{-\pi b u} \mathrm{f}_{s_{2}} \otimes \mathrm{e}_{s_{1}}+e^{-\pi b u} \mathrm{k}_{s_{2}}^{-1} \otimes \mathrm{k}_{s_{1}}+e^{\pi b u} \mathrm{k}_{s_{2}} \otimes \mathrm{k}_{s_{1}}^{-1} .
\end{aligned}
$$

The verification of the relations $(\mathrm{D} .17$ is easy. Introducing

$$
\mathrm{r}_{s_{2} s_{1}}(u)=\mathrm{R}_{s_{2} s_{1}}^{-1} \cdot \mathrm{R}_{s_{2} s_{1}}(u),
$$


it follows from (D.8) that (D.17) is equivalent to the system of equations

$$
\mathrm{r}_{s_{2} s_{1}}(u) \Delta(\mathrm{X})=\Delta(\mathrm{X}) \mathrm{r}_{s_{2} s_{1}}(u) \text {. }
$$

Validity of the relation (D.24) follows from $[s, \Delta(X)]=0$.

The verification of the relations (D.18) is somewhat harder. To begin with, let us observe that it suffices to verify relation (D.20), say.

Lemma 9. Validity of (D.18) for $(i, j)=(1,2)$ implies that $\mathrm{R}_{s_{2} s_{1}}(u)$ satisfies the three remaining relations in (D.18) as well.

Proof. Equivalence of the $(1,2)$ and $(2,1)$ equations in (D.18) can be established by invoking the automorphism $\theta$, defined in (37). Introduce $\tau_{\theta} \equiv(\theta \otimes \theta) \circ \tau$. It is easy to see that $\tau_{\theta}\left(\mathrm{R}_{s_{2} s_{1}}\right)=\mathrm{R}_{s_{2} s_{1}}$ and $\tau_{\theta}(\Delta(C))=\Delta(C)$. Therefore, $\tau_{\theta}\left(\mathrm{R}_{s_{2} s_{1}}(u)\right)=\mathrm{R}_{s_{2} s_{1}}(u)$. The claimed equivalence of the $(1,2)$ and $(2,1)$ equations in $\left(\mathrm{D} .18\right.$ follows now by observing that $\tau_{\theta}\left(\ell_{12}(u)\right)=\ell_{21}(u)$.

In order to prove that relation (D.18) for $(i, j)=(1,1)$ follows from the validity of relations (D.18) for $(i, j)=(1,2),(i, j)=(2,1)$ and (D.17), let us consider the following object:

$$
\begin{aligned}
\mathrm{X}(u) & =q\left(\mathrm{k}_{s_{2}}^{2} \otimes \mathrm{k}_{s_{1}}^{2}-\mathrm{k}_{s_{2}}^{-2} \otimes \mathrm{k}_{s_{1}}^{-2}\right) \ell_{11}(u)+\mathrm{k}_{s_{2}} \otimes \mathrm{k}_{s_{1}}\left(e^{-\pi b u} \mathrm{C}_{s_{2}} \otimes 1+e^{\pi b u} 1 \otimes \mathrm{C}_{s_{1}}\right) \\
& +\mathrm{k}_{s_{2}}^{-1} \otimes \mathrm{k}_{s_{1}}^{-1}\left(e^{\pi b u} \mathrm{C}_{s_{2}} \otimes 1+e^{-\pi b u} 1 \otimes \mathrm{C}_{s_{1}}\right) .
\end{aligned}
$$

The operator $\left(\mathrm{k}_{s_{2}}^{2} \otimes \mathrm{k}_{s_{1}}^{2}-\mathrm{k}_{s_{2}}^{-2} \otimes \mathrm{k}_{s_{1}}^{-2}\right)$ does not have a normalizable zero mode. Validity of relation (D.18) for $(i, j)=(1,1)$ therefore follows from

$$
\mathrm{R}_{s_{2} s_{1}}(u) \mathrm{X}(u)=\tau(\mathrm{X}(-u)) \mathrm{R}_{s_{2} s_{1}}(u) .
$$

Validity of this relation follows from the observation that $\mathrm{X}(u)$ can be represented in the form

$$
\mathrm{X}(u)=\left(\mathrm{k}_{s_{2}} \otimes \mathrm{k}_{s_{1}}\right) \ell_{12}(u)\left(\pi_{s_{2}} \otimes \pi_{s_{1}}\right) \Delta(e)-\left(\mathrm{k}_{s_{2}}^{-1} \otimes \mathrm{k}_{s_{1}}^{-1}\right) \ell_{21}(u)\left(\pi_{s_{2}} \otimes \pi_{s_{1}}\right) \Delta(f) .
$$

Exchanging $\mathrm{k} \leftrightarrow \mathrm{k}^{-1}$ in (D.27), we can derive relation (D.18) for $(i, j)=(2,2)$ in a completely analogous way.

It remains to verify relation $(\mathrm{D} .18$ for $(i, j)=(1,2)$. This relation may be rewritten in terms of the operator $\mathrm{r}_{s_{2} s_{1}}(u)$ defined in (D.23) by using the relation (D.9). We conclude that validity of (D.18) follows from the validity of

$$
\mathrm{r}_{s_{2} s_{1}}(u)\left(e^{2 \pi b u} \mathrm{O}_{1}+\mathrm{O}_{2}\right)=\left(e^{2 \pi b u} \mathrm{O}_{1}+\mathrm{O}_{3}\right) \mathrm{r}_{s_{2} s_{1}}(u),
$$

where we have introduced the convenient abbreviations

$$
\begin{aligned}
& \mathrm{O}_{1} \equiv \mathrm{e}_{s_{2}} \otimes \mathrm{k}_{s_{1}}^{-1}, \\
& \mathrm{O}_{2} \equiv \mathrm{k}_{s_{2}} \otimes \mathrm{e}_{s_{1}},
\end{aligned}
$$

In order to prove that $r_{s_{2} s_{1}}(u)$ satisfies (D.28) it will be convenient to use the Clebsch-Gordan maps $\mathrm{C}_{s_{2} s_{1}}$ introduced in Subsection D.1 Let us observe that (D.24) implies that the $\mathrm{r}-$ matrix is diagonalized by the Clebsch-Gordan maps:

$$
\mathrm{C}_{s_{2} s_{1}} \cdot \mathrm{r}_{s_{2} s_{1}}(u)=r_{s_{2} s_{1}}^{\mathrm{s}}(u) \cdot \mathrm{C}_{s_{2} s_{1}}
$$

where $r_{s_{2} s_{1}}^{\mathrm{s}}(u)$ is the operator on $\mathcal{M}$ associated to the scalar function $r_{s_{2} s_{1}}^{s}(u)$ via (D.2).

In order to further evaluate equation (D.28) we will need to describe the images $\tilde{O}_{\ell}, \ell=1,2,3$ of the operators $\mathrm{O}_{\ell}$ under the maps $\mathrm{C}_{s_{2} s_{1}}$ which are defined by $\mathrm{C}_{s_{2} s_{1}} \cdot \mathrm{O}_{\ell} \equiv \tilde{\mathrm{O}}_{\ell} \cdot \mathrm{C}_{s_{2} s_{1}}$. 
Proposition 4. The operators $\tilde{\mathrm{O}}_{\ell}$ can be represented as follows: If $g \equiv\left(g_{s} ; s \in \mathbb{R}^{+}\right) \in \mathcal{M}$ then $\tilde{\mathrm{O}}_{\ell} g \equiv\left(\left(\tilde{\mathrm{O}}_{\ell} g\right)_{s} ; s \in \mathbb{R}^{+}\right)$, where

$$
\left(\tilde{\mathrm{O}}_{\ell} g\right)_{s}(k) \equiv \sum_{\nu=-1}^{1} A_{\ell ; s}^{\nu ; s_{2} s_{1}}(k) \mathrm{T}_{s}^{\nu} g_{s}(k+i b),
$$

where $\mathrm{T}_{s}^{\nu} g_{s}=g_{s+i \nu b}$. The coefficients $A_{\ell ; s}^{\nu ; s_{2} s_{1}}(k)$ are symmetric under exchange of $s_{2}$ and $s_{1}$,

$$
A_{\ell ; s}^{\nu ; s_{2} s_{1}}(k)=A_{\ell ; s}^{\nu ; s_{1} s_{2}}(k)
$$

and are otherwise related to each other by

$$
\begin{aligned}
& A_{2 ; s}^{\nu ; s_{2} s_{1}}(k)=e^{+2 \pi b \nu s+i \pi b^{2} \nu^{2}} A_{1 ; s}^{\nu ; s_{1} s_{2}}(k), \\
& A_{3 ; s}^{\nu ; s_{2} s_{1}}(k)=e^{-2 \pi b \nu s-i \pi b^{2} \nu^{2}} A_{1 ; s}^{\nu ; s_{1} s_{2}}(k) .
\end{aligned}
$$

The proof of Proposition 4 which is somewhat technical, is given in Subsection (D.5).

Proposition 4 together with equation (D.30) allows us to rewrite the defining relation (D.28) as a commutation relation satisfied by the corresponding operators on $\mathcal{M}$. Applying (D.28) to a function $g_{s}$ and matching the coefficients in front of $g_{s+i \nu}(k+i b)$ in the resulting equation, we derive functional equations on $r_{s_{2} s_{1}}^{s}(u)$,

$$
\begin{aligned}
r_{s_{2} s_{1}}^{s+i b \nu}(u) & \left(e^{2 \pi b u} A_{1 ; s}^{\nu ; s_{2} s_{1}}(k)+A_{2 ; s}^{\nu ; s_{2} s_{1}}(k)\right) \\
& =\left(e^{2 \pi b u} A_{1 ; s}^{\nu ; s_{2} s_{1}}(k)+A_{3 ; s}^{\nu ; s_{2} s_{1}}(k)\right) r_{s_{2} s_{1}}^{s}(u) .
\end{aligned}
$$

The case $\nu=0$ holds trivially. Taking into account (D.32) and (D.33), Eqs. (D.34) for $\nu= \pm 1$ are equivalent to a single functional equation,

$$
r_{s_{2} s_{1}}^{s+i b}(u)\left(e^{2 \pi b u}+\zeta\right)=\left(e^{2 \pi b u}+\zeta^{-1}\right) r_{s_{2} s_{1}}^{s}(u),
$$

where $\zeta=e^{i \pi b^{2}+2 \pi b s}$. In terms of $\tilde{r}_{s_{2} s_{1}}^{s}(u)=e^{-i \pi s^{2}} r_{s_{2} s_{1}}^{s}(u)$ one may rewrite this functional relation as follows

$$
\tilde{r}_{s_{2} s_{1}}^{s+i \frac{b}{2}}(u) \cosh \pi b(s+u)=\tilde{r}_{s_{2} s_{1}}^{s-i \frac{b}{2}}(u) \cosh \pi b(s-u) .
$$

Recalling that the special function $w_{b}(x)$ satisfies $\mathrm{A} .8$, we conclude that equation (D.35) is solved by the expression for $r_{s_{2} s_{1}}^{s}$ which follows from eqn. (D.14) via (D.23) and (D.30), namely

$$
r_{s_{2} s_{1}}^{s}(u)=e^{\pi i\left(s^{2}-s_{1}^{2}-s_{2}^{2}-\frac{Q^{2}}{4}\right)} \frac{w_{b}(u+s)}{w_{b}(s-u)}=e^{\pi i\left(s^{2}-s_{1}^{2}-s_{2}^{2}-\frac{Q^{2}}{4}\right)} D_{u}(s),
$$

where $D_{\alpha}(x)$ is defined in A.19.

Property A.24) implies that $\left|r_{s_{2} s_{1}}^{s}(u)\right|=1$ if $u, s \in \mathbb{R}$. Since $\mathrm{s}_{21}$ is self-adjoint, we infer that $r_{s_{2} s_{1}}^{\mathrm{s}}(u)$ is a unitary operator for $u \in \mathbb{R}$. On the other hand property A.10 implies that $g_{b}(x)$ given by (D.7) satisfies $\left|g_{b}(x)\right|=1$ for $x \in \mathbb{R}^{+}$. Since $\mathrm{e}_{2} \otimes \mathrm{f}_{\mathbf{1}}$ in (D.6) is positive self-adjoint, we infer that $\mathrm{R}_{s_{2} s_{1}}$ is unitary. The unitarity of $\mathrm{R}_{s_{2} s_{1}}(u)$ for $u \in \mathbb{R}$ follows.

Let us finally remark that relation A.26 leads to the following asymptotics of $\mathrm{R}_{s_{2} s_{1}}(u)$

$$
\begin{array}{ll}
\mathrm{R}_{s_{2} s_{1}}(u) \sim e^{-i \pi\left(u^{2}+\ldots\right)} \mathrm{R}_{s_{2} s_{1}} & \text { for } \operatorname{Re}(u) \rightarrow+\infty \\
\mathrm{R}_{s_{2} s_{1}}(u) \sim e^{+i \pi\left(u^{2}+\ldots\right)} \overline{\mathrm{R}}_{s_{2} s_{1}} & \text { for } \operatorname{Re}(u) \rightarrow-\infty,
\end{array}
$$

where $\overline{\mathrm{R}}_{s_{2} s_{1}} \equiv \mathrm{P} \cdot \mathrm{R}_{s_{2} s_{1}}^{-1} \cdot \mathrm{P}$. 


\section{D.4 Clebsch-Gordan and Racah-Wigner coefficients for $\mathcal{P}_{s}$}

In order to prove Proposition 4 we will need to describe the Clebsch-Gordan maps $\mathrm{C}_{s_{2} s_{1}}$ more explicitly. For the following it will be convenient to use the variables $\alpha_{r}=\frac{Q}{2}+i s_{r}$ in order parameterize the representations $\mathcal{P}_{s_{r}}, r=0,1,2 \ldots$ The Clebsch-Gordan maps $\mathrm{C}_{s_{2} s_{1}}$ can then be represented explicitly as an integral transformation [PT2] BT]:

$$
\tilde{f}_{s}(k) \equiv \int d k_{2} d k_{1}\left[\begin{array}{c|cc}
\alpha_{3} & \alpha_{2} & \alpha_{1} \\
k_{3} & k_{2} & k_{1}
\end{array}\right] f\left(k_{2}, k_{1}\right)
$$

The kernel which appears on the right hand side, the so-called b-Clebsch-Gordan kernel, was calculated in [PT2, BT]. It is of the general form

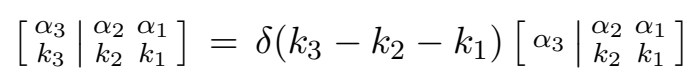

where the function $\left[\begin{array}{l|ll}\alpha_{3} & \alpha_{2} & \alpha_{1} \\ k_{2} & k_{1}\end{array}\right]$ is given as

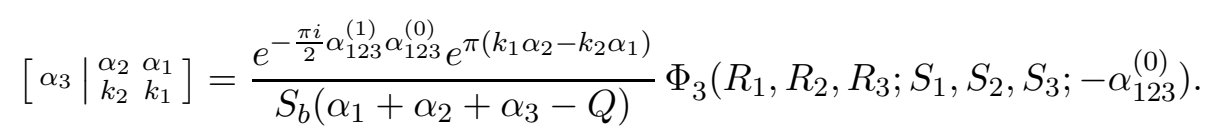

In equation (D.40) we have used the special function $\Phi_{3}\left(R_{1}, R_{2}, R_{3} ; S_{1}, S_{2}, S_{3} ; x\right)$ defined in A.14 whose arguments have been chosen as follows:

$$
\begin{array}{ll}
R_{1}=\alpha_{1}+i k_{1} & S_{1}=\alpha_{123}^{(1)}+\alpha_{1}+i k_{1} \\
R_{2}=\alpha_{2}-i k_{2} & S_{2}=\alpha_{123}^{(2)}+\alpha_{2}-i k_{2} \\
R_{3}=\alpha_{123}^{(0)} & S_{3}=Q .
\end{array}
$$

Here and below we are using the following notations:

$$
\begin{aligned}
& \alpha_{i j k}^{(1)}=\alpha_{j}+\alpha_{k}-\alpha_{i}, \\
& \alpha_{i j k}^{(2)}=\alpha_{k}+\alpha_{i}-\alpha_{j}, \quad \alpha_{i j k}^{(0)}=\alpha_{i}+\alpha_{j}+\alpha_{k}-Q . \\
& \alpha_{i j k}^{(3)}=\alpha_{i}+\alpha_{j}-\alpha_{k},
\end{aligned}
$$

The precise relation to the $\mathrm{b}-\mathrm{Clebsch}-$ Gordan coefficients from $[\overline{\mathrm{BT}}]$ is as follows:

$$
\left[\begin{array}{l|ll}
\alpha_{3} & \alpha_{2} & \alpha_{1} \\
k_{3} & k_{2} & k_{1}
\end{array}\right]_{\text {here }} \equiv \nu_{\alpha_{2} \alpha_{1}}^{\alpha_{3}}\left[\begin{array}{l|ll}
\alpha_{3} & \alpha_{2} & \alpha_{1} \\
k_{3} & k_{2} & k_{1}
\end{array}\right]_{\mathrm{BT}}
$$

with phases $\nu_{\alpha_{2} \alpha_{1}}^{\alpha_{3}}$ chosen as

$$
\left(\nu_{\alpha_{2} \alpha_{1}}^{\alpha_{3}}\right)^{-1}=S_{b}\left(\alpha_{3}+\alpha_{1}-\alpha_{2}\right) S_{b}\left(\alpha_{3}+\alpha_{2}-\alpha_{1}\right) .
$$

The b-Racah-Wigner coefficients are then defined by the relation

$$
\begin{aligned}
& {\left[\begin{array}{l|ll}
\alpha_{s} & \alpha_{1} & \alpha_{0} \\
k_{s} & k_{1} & k_{0}
\end{array}\right]\left[\begin{array}{l|ll}
\alpha_{3} & \alpha_{2} & \alpha_{s} \\
k_{3} & k_{2} & k_{s}
\end{array}\right]} \\
& =\frac{1}{i} \int_{\mathbb{S}} d \alpha_{t}\left\{\begin{array}{ll|l}
\alpha_{0} & \alpha_{1} & \alpha_{s} \\
\alpha_{2} & \alpha_{3} & \alpha_{t}
\end{array}\right\}\left[\begin{array}{l|ll}
\alpha_{3} & \alpha_{t} & \alpha_{0} \\
k_{3} & k_{t} & k_{0}
\end{array}\right]\left[\begin{array}{l|ll}
\alpha_{t} & \alpha_{2} & \alpha_{1} \\
k_{t} & k_{2} & k_{1}
\end{array}\right],
\end{aligned}
$$


where $\mathbb{S} \equiv \frac{Q}{2}+i \mathbb{R}_{+}$. The coefficients $\{\ldots\}$ can be represented explicitly by the following formula

$$
\begin{aligned}
\left\{\begin{array}{cc|c}
\alpha_{0} & \alpha_{1} \\
\alpha_{2} & \alpha_{3} & \alpha_{s} \\
\alpha_{t}
\end{array}\right\}= & \frac{S_{b}\left(\alpha_{t}+\alpha_{0}+\alpha_{3}-Q\right) S_{b}\left(\alpha_{3}+\alpha_{0}-\alpha_{t}\right) S_{b}\left(\alpha_{3}+\alpha_{t}-\alpha_{0}\right)}{S_{b}\left(\alpha_{s}+\alpha_{2}+\alpha_{3}-Q\right) S_{b}\left(\alpha_{3}+\alpha_{2}-\alpha_{s}\right) S_{b}\left(\alpha_{3}+\alpha_{s}-\alpha_{2}\right)} \\
& \times \frac{S_{b}\left(\alpha_{t}+\alpha_{2}-\alpha_{1}\right)}{S_{b}\left(\alpha_{s}+\alpha_{0}-\alpha_{1}\right)} \Phi_{4}\left(U_{1}, U_{2}, U_{3}, U_{4} ; V_{1}, V_{2}, V_{3}, V_{4} ; 0\right)
\end{aligned}
$$

where

$$
\begin{array}{ll}
U_{1}=\alpha_{s}+\alpha_{0}-\alpha_{1} & V_{1}=Q-\alpha_{t}+\alpha_{s}+\alpha_{3}-\alpha_{1} \\
U_{2}=\alpha_{s}+Q-\alpha_{0}-\alpha_{1} & V_{2}=\alpha_{t}+\alpha_{s}+\alpha_{3}-\alpha_{1} \\
U_{3}=\alpha_{s}+\alpha_{2}+\alpha_{3}-Q & V_{3}=2 \alpha_{s} \\
U_{4}=\alpha_{s}+\alpha_{3}-\alpha_{2} & V_{4}=Q .
\end{array}
$$

For completeness let us note that the b-Racah-Wigner coefficients $\{\cdots\}$ are related to the corresponding objects from [PT2] via

$$
\left\{\begin{array}{ll|l}
\alpha_{0} & \alpha_{1} & \alpha_{s} \\
\alpha_{2} & \alpha_{3} & \alpha_{t}
\end{array}\right\}_{\text {here }}=\frac{\nu_{\alpha_{1} \alpha_{0}}^{\alpha_{s}} \nu_{\alpha_{2} \alpha_{s}}^{\alpha_{3}}}{\nu_{\alpha_{2} \alpha_{1}}^{\alpha_{t}} \nu_{\alpha_{t} \alpha_{0}}^{\alpha_{3}}}\left\{\begin{array}{cc|c}
\alpha_{0} & \alpha_{1} & \alpha_{s} \\
\alpha_{2} & \alpha_{3} & \alpha_{t}
\end{array}\right\}_{\mathrm{PT} 2}
$$

The b-Clebsch-Gordan and b-Racah-Wigner coefficients are meromorphic functions of all of their arguments. The complete set of poles may be described as follows [PT2 [BT] ${ }^{7}$

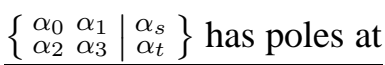

$$
\begin{array}{ll}
Q-\alpha_{32 s}^{(\iota)}=-n b-m b^{-1}, & \alpha_{3 t 0}^{(\iota)}=-n b-m b^{-1} \\
Q-\alpha_{s 10}^{(\iota)}=-n b-m b^{-1}, & \alpha_{t 21}^{(\iota)}=-n b-m b^{-1}
\end{array}
$$

where $n, m \in \mathbb{Z}^{\geq 0}$.

$\underline{\left[\begin{array}{l|ll}\alpha_{3} & \alpha_{2} & \alpha_{1} \\ k_{3} & k_{2} & k_{1}\end{array}\right] \text { has poles at }}$

$$
\begin{aligned}
& Q-\alpha_{321}^{(\iota)}=-n b-m b^{-1}, \quad \iota=0,1,2,3, \\
& \pm i k_{i}=\alpha_{i}+n b+m b^{-1}, \quad i=1,2, \\
& \pm i k_{3}=Q-\alpha_{3}+n b+m b^{-1},
\end{aligned}
$$

where again $n, m \in \mathbb{Z}^{\geq 0}$.

\section{D.5 Proof of Proposition 4}

Our proof of Proposition 4 will be based on the following nontrivial identity satisfied by the b-Clebsch-Gordan kernel.

Lemma 10.

$$
\begin{aligned}
& {\left[[ \begin{array} { l | l } 
{ \alpha _ { 1 } } & { \begin{array} { c c } 
{ \alpha _ { 1 } } & { - b } \\
{ k _ { 1 } } & { i b }
\end{array} ] }
\end{array} ] \left[\begin{array}{l|l}
\alpha_{3} & \left.\begin{array}{ll}
\alpha_{2} & \alpha_{1} \\
k_{2} & k_{1}+i b
\end{array}\right]
\end{array}\right.\right.}
\end{aligned}
$$

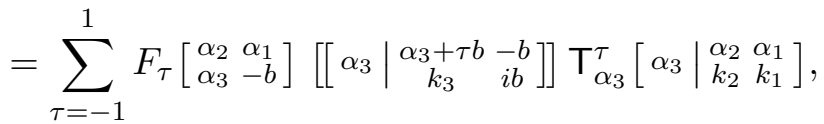

\footnotetext{
${ }^{7}$ We take the opportunity to correct some typos in these references.
} 
where $\mathrm{T}_{\alpha_{3}}^{\tau} f\left(\alpha_{3}\right)=f\left(\alpha_{3}+\tau b\right), k_{3}=k_{2}+k_{1}$ and we have furthermore used the notation

$$
\begin{aligned}
& {\left[\left[\begin{array}{c|cc}
\alpha_{3} & \alpha_{2} & \alpha_{1} \\
k_{2} & -i \alpha_{1}
\end{array}\right]\right]=2 \pi i \operatorname{Res}_{k_{1}=-i \alpha_{1}}\left[\begin{array}{l|l|l}
\alpha_{3} & \alpha_{2} & \alpha_{1} \\
k_{2} & k_{1}
\end{array}\right],} \\
& F_{\tau}\left[\begin{array}{cc}
\alpha_{2} & \alpha_{1} \\
\alpha_{3} & -b
\end{array}\right]=2 \pi i \underset{\alpha_{\tau}=\alpha_{3}+b \tau}{\operatorname{Res}}\left\{\begin{array}{lll}
-b & \alpha_{1} & \alpha_{1} \\
\alpha_{2} & \alpha_{3} & \alpha_{\tau}
\end{array}\right\},
\end{aligned}
$$

We have the explicit formulae

$$
\begin{aligned}
{\left[\left[\alpha_{3} \mid \begin{array}{cc}
\alpha_{2} & \alpha_{1} \\
k_{2} & -i \alpha_{1}
\end{array}\right]=\right.} & \frac{e^{\frac{\pi i}{2}\left(\Delta_{\alpha_{3}}-\Delta_{\alpha_{2}}-\Delta_{\alpha_{1}}\right)}}{S_{b}\left(\alpha_{3}+\alpha_{2}-\alpha_{1}\right)} e^{-\pi k_{2} \alpha_{1}} \frac{S_{b}\left(\alpha_{2}-i k_{2}\right)}{S_{b}\left(\alpha_{1}+\alpha_{3}-i k_{2}\right)}, \\
F_{-}\left[\begin{array}{cc}
\alpha_{2} & \alpha_{1} \\
\alpha_{3} & -b
\end{array}\right]= & \frac{S_{b}\left(2 \alpha_{3}-2 b-Q\right)}{S_{b}\left(2 \alpha_{1}+b\right)} \sin \pi b\left(\alpha_{2}+\alpha_{1}-\alpha_{3}\right), \\
F_{+}\left[\begin{array}{cc}
\alpha_{2} & \alpha_{1} \\
\alpha_{3} & -b
\end{array}\right]= & \frac{S_{b}\left(2 \alpha_{3}-Q\right)}{S_{b}\left(2 \alpha_{1}+b\right)} \sin \pi b\left(\alpha_{3}+\alpha_{2}+\alpha_{1}-Q\right) \\
& \times \sin \pi b\left(\alpha_{3}+\alpha_{1}-\alpha_{2}\right) \sin \pi b\left(\alpha_{3}+\alpha_{2}-\alpha_{1}\right),
\end{aligned}
$$

where $\Delta_{\alpha}=\alpha(Q-\alpha)$.

Given that Lemma 10 holds, it becomes easy to complete the proof of Proposition 4 as follows: Notice that the left hand side of (D.49) can be written as

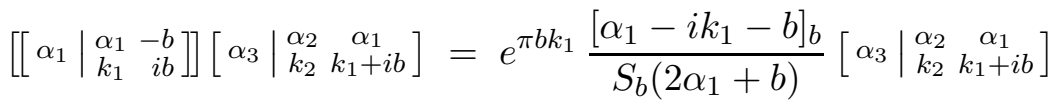

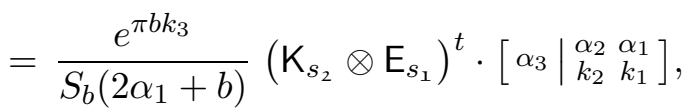

where $\mathrm{O}^{t}$ denotes the transpose of an operator on $\mathcal{P}_{s_{2}} \otimes \mathcal{P}_{s_{1}}$ defined by

$$
\int d k_{2} d k_{1} f\left(k_{2}, k_{1}\right)(\mathrm{O} g)\left(k_{2}, k_{1}\right) \equiv \int d k_{2} d k_{1}\left(\mathrm{O}^{t} f\right)\left(k_{2}, k_{1}\right) g\left(k_{2}, k_{1}\right) .
$$

Equation (D.49) may therefore be written in the form

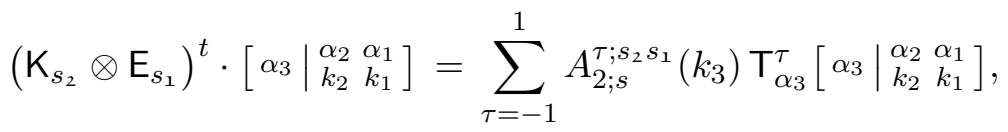

where

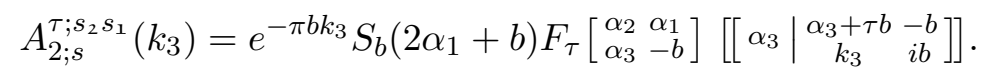

By using the explicit expressions D.51, D.52 one may easily verify that the coefficients $A_{2 ; s}^{t ; s_{2} s_{1}}$ are symmetric under the exchange of $s_{2}$ and $s_{1}$, as claimed. This completes the proof of all the relevant statements of Proposition 4 for the case of the operator $\mathrm{O}_{2}$.

In order to cover the remaining cases let us observe that

$$
\begin{aligned}
& \mathrm{O}_{2} \equiv \mathrm{K}_{s_{2}} \otimes \mathrm{E}_{s_{1}}=\mathrm{B}_{s_{1} s_{2}} \mathrm{O}_{1} \mathrm{~B}_{s_{1} s_{2}}^{-1}, \\
& \mathrm{O}_{3} \equiv \mathrm{R}_{s_{2} s_{1}}^{-1}\left(\mathrm{~K}_{s_{2}}^{-1} \otimes \mathrm{E}_{s_{1}}\right) \mathrm{R}_{s_{2} s_{1}}=\mathrm{B}_{s_{2} s_{1}}^{-1} \mathrm{O}_{1} \mathrm{~B}_{s_{2} s_{1}},
\end{aligned}
$$

where $\mathrm{B}=\mathrm{PR}$ is the braiding operator. 
Therefore, invoking Proposition 3 , we conclude that $\mathrm{O}_{1}$ and $\mathrm{O}_{3}$ also satisfy Proposition 4 with coefficients $A_{r ; s}^{\nu ; s_{2} s_{1}}(k), r=1,2,3$ being related by

$$
A_{2 ; s}^{\nu ; s_{2} s_{1}}(k)=\frac{\Omega_{s_{1} s_{2}}^{s+i b \nu}}{\Omega_{s_{1} s_{2}}^{s}} A_{1 ; s}^{\nu ; s_{1} s_{2}}(k), \quad A_{3 ; s}^{\nu ; s_{2} s_{1}}(k)=\frac{\Omega_{s_{2} s_{1}}^{s}}{\Omega_{s_{2} s_{1}}^{s+i b \nu}} A_{1 ; s}^{\nu ; s_{1} s_{2}}(k),
$$

respectively. The proof of Proposition 4 is complete.

Proof of Lemma 10. Our starting point is the defining relation for the b-Racah-Wigner symbols, equation (D.44). Our claim will follow from (D.44) as an identity satisfied by the residues of the meromorphic continuation of (D.44). We need to analyze the relevant limits step by step.

$\underline{U_{1} \equiv \alpha_{s}+\alpha_{0}-\alpha_{1} \rightarrow-b:}$

Note that in the limit $U_{1} \equiv \alpha_{s}+\alpha_{0}-\alpha_{1} \rightarrow-b$ the contour of integration in the definition of $\Phi_{4}$, eqn. A.14, gets pinched between the poles of the integrand $s=Q-V_{4} \equiv 0$ and $s=-U_{1}-b$ as well as between $s=Q-V_{4}+b \equiv b$ and $s=-U_{1}$. This implies that $\Phi_{4}$ has a pole when $U_{1}=-b$. In order extract the part which gets singular in the limit under consideration one may deform the contour of integration in A.14 to the sum of two circles around $s=0$ and $s=b$ plus a contour which passes to the right of the pole at $s=b$ and which approaches the imaginary axis at infinity. The residue is given as

$$
-\frac{1}{2 \pi} \sin \pi b^{2} \frac{S_{b}\left(U_{2}\right) S_{b}\left(U_{3}\right) S_{b}\left(U_{4}\right)}{S_{b}\left(V_{1}\right) S_{b}\left(V_{2}\right) S_{b}\left(V_{3}\right)}\left(1+\frac{\sin \pi b U_{2} \sin \pi b U_{3} \sin \pi b U_{4}}{\sin \pi b V_{1} \sin \pi b V_{2} \sin \pi b V_{3}}\right) .
$$

Considering the behavior of $\{\cdots\}$ at $U_{1}=-b$, one finds that the pole of $\Phi_{4}$ at $U_{1}=-b$ is canceled by the zero from the prefactor $\left(S_{b}\left(\alpha_{s}+\alpha_{0}-\alpha_{1}\right)\right)^{-1}$. Taken together one obtains the following special value for $\{\cdots\}$ at $\alpha_{s}+\alpha_{0}-\alpha_{1}=-b$ :

$$
\begin{aligned}
\left\{\begin{array}{cc|c}
\alpha_{0} & \alpha_{1} \\
\alpha_{2} & \alpha_{3} & \alpha_{2}-\alpha_{1}-b \\
\alpha_{t}
\end{array}\right\}= & \frac{S_{b}\left(Q-2 \alpha_{0}-b\right)}{S_{b}\left(2\left(\alpha_{1}-\alpha_{0}-b\right)\right)} \frac{S_{b}\left(\alpha_{t}+\alpha_{2}-\alpha_{1}\right)}{S_{b}\left(\alpha_{3}+\alpha_{2}-\alpha_{1}+\alpha_{0}+b\right)} \\
& \frac{S_{b}\left(\alpha_{3}+\alpha_{t}-\alpha_{0}\right)}{S_{b}\left(\alpha_{3}+\alpha_{t}-\alpha_{0}-b\right)} \frac{S_{b}\left(\alpha_{3}+\alpha_{0}-\alpha_{t}\right)}{S_{b}\left(Q+\alpha_{3}-\alpha_{t}-\alpha_{0}-b\right)} \\
& S_{b}\left(\alpha_{3}+\alpha_{0}+\alpha_{t}-Q\right)\left(1+\frac{\sin \pi b U_{2} \sin \pi b U_{3} \sin \pi b U_{4}}{\sin \pi b V_{1} \sin \pi b V_{2} \sin \pi b V_{3}}\right) .
\end{aligned}
$$

The parameters $U_{2}, U_{3}, U_{4}$ and $V_{1}, V_{2}, V_{3}$ are now given by

$$
\begin{array}{ll}
U_{2}=Q-2 \alpha_{0}-b & V_{1}=Q-\alpha_{t}+\alpha_{3}-\alpha_{0}-b \\
U_{3}=\alpha_{1}-\alpha_{0}-b+\alpha_{3}+\alpha_{2}-Q & V_{2}=\alpha_{t}+\alpha_{3}-\alpha_{0}-b \\
U_{4}=\alpha_{1}-\alpha_{0}-b+\alpha_{3}-\alpha_{2} & V_{3}=2\left(\alpha_{1}-\alpha_{0}-b\right) .
\end{array}
$$

$\underline{k_{0} \rightarrow i \alpha_{0}}:$

In the same way as in the previous paragraph one may show that the b-Clebsch-Gordan coefficients $\left[\begin{array}{l|ll}\alpha_{s} & \alpha_{1} & \alpha_{0} \\ k_{s} & k_{1} & k_{0}\end{array}\right]$ and $\left[\begin{array}{l|ll}\alpha_{3} & \alpha_{t} & \alpha_{0} \\ k_{3} & k_{t} & k_{0}\end{array}\right]$ develop poles, with residues given by (D.50).

Continuation to $\operatorname{Re}\left(\alpha_{0}\right)=-b$ with $\operatorname{Re}\left(\alpha_{3}\right)=\frac{Q}{2}-\delta, 0<\delta<b<b^{-1}$.

The left hand side of D.44 is analytic in the range under consideration. In order to describe the analytic continuation of the right hand side let us note that in the continuation from $\operatorname{Re}\left(\alpha_{0}\right)=Q / 2$ 
to $\operatorname{Re}\left(\alpha_{0}\right)=-b$ exactly three poles $\alpha_{t}=\alpha_{t}^{(k)}, k=-1,0,1$ cross the contour of integration, namely

$$
\begin{aligned}
k=-1: & \alpha_{t}^{(k)}=\alpha_{3}+\alpha_{0}, \\
k=0: & \alpha_{t}^{(k)}=\alpha_{3}, \\
k=1: & \alpha_{t}^{(k)}=\alpha_{3}-\alpha_{0} .
\end{aligned}
$$

The analytic continuation of the right hand side of (D.44) may therefore be represented by replacing the integration contour $\mathbb{S}$ in $(\mathrm{D} .44)$ by $\mathcal{C}=\mathbb{S} \cup \bigcup_{k=-1}^{1} \mathcal{C}_{k}$, with $\mathcal{C}_{t}$ being a small circle around the poles at $\alpha_{t}=\alpha_{t}^{(k)}$.

$\underline{\text { Limit } \alpha_{1} \rightarrow-b \text { with } \operatorname{Re}\left(\alpha_{3}\right)=\frac{Q}{2}-\delta, 0<\delta<b<b^{-1}}$.

We observe that the integral over $\mathbb{S}$ vanishes due to the factor $S_{b}\left(Q-2 \alpha_{1}-b\right)$. This is not the case for contributions from the poles $\alpha_{t}=\alpha_{t}^{(k)}, k=-1,0,1$. Our claim now follows by straightforward computations.

\section{E Construction of the Q-operator $\mathrm{Q}(u)$}

\section{E.1 Preliminaries}

Let us now enter into the construction of the Q-operators. We begin by collecting some useful preliminaries. We will work in the Schrödinger representation where the operators $\mathrm{x}_{r}, r=1, \ldots, \mathrm{N}$ are diagonal. We will need operators $\mathrm{U}, \Omega$, and $\mathrm{J}_{s}$ defined in the Schrödinger representation by the following integral kernels

$$
\begin{gathered}
U\left(\mathbf{x}, \mathbf{x}^{\prime}\right)=\prod_{r=1}^{\mathrm{N}} \delta\left(x_{r+1}-x_{r}^{\prime}\right), \quad \Omega\left(\mathbf{x}, \mathbf{x}^{\prime}\right)=\prod_{r=1}^{\mathrm{N}} \delta\left(x_{r}+x_{r}^{\prime}\right), \\
J_{s}\left(\mathbf{x}, \mathbf{x}^{\prime}\right)=\left(w_{b}\left(i \frac{Q}{2}-2 s\right)\right)^{\mathrm{N}} \prod_{r=1}^{\mathrm{N}} D_{s-\frac{i}{2} Q}\left(x_{r}-x_{r}^{\prime}\right) .
\end{gathered}
$$

$\mathrm{U}$ is the cyclic shift operator defined in $(82) . \Omega$ and $\mathrm{J}_{s}$ are products of local operators,

$$
\Omega=\prod_{r=1}^{\mathrm{N}} \Omega_{r}, \quad \mathrm{~J}_{s}=\prod_{r=1}^{\mathrm{N}} \mathrm{j}_{r} .
$$

Here $\mathrm{j}_{r}$ is the operator which intertwines at the site $r$ the representations $\mathcal{P}_{s}$ and $\mathcal{P}_{-s}$ of $\mathcal{U}_{q}(\mathfrak{s l}(2, \mathbb{R}))$ (see [PT2]), and $\Omega_{r}$ is the operator which realizes at the site $r$ the parity operation: $\Omega_{r} f\left(x_{1}, x_{2}, \ldots, x_{r}, \ldots, x_{\mathrm{N}}\right)=f\left(x_{1}, x_{2}, \ldots,-x_{r}, \ldots, x_{\mathrm{N}}\right)$ (whence $\Omega_{r} \mathrm{p}_{r} \Omega_{r}=\theta\left(\mathrm{p}_{r}\right)$ and $\Omega_{r} \mathrm{x}_{r} \Omega_{r}=\theta\left(\mathrm{x}_{r}\right)$, where the automorphism $\theta$ is defined by (36) $)$.

We will denote the standard scalar product on $L^{2}(\mathbb{R})$ by $\langle f \mid g\rangle=\int_{\mathbb{R}} d \mathbf{x} f(\mathbf{x}) g(\mathbf{x})$. For a given operator $\mathrm{O}$, its transposed $\mathrm{O}^{t}$ and hermitian-conjugated $\mathrm{O}^{*}$ are defined, respectively, by (the bar denotes complex conjugation)

$$
\left\langle\mathrm{O}^{t} f \mid g\right\rangle=\langle f \mid \mathrm{O} g\rangle, \quad\left\langle\overline{\mathrm{O}^{*} f} \mid g\right\rangle=\langle\bar{f} \mid \mathrm{O} g\rangle .
$$

This definition extends to a matrix with operator-valued coefficients as follows

$$
\left(L^{t}\right)_{i j}=\left(L_{i j}\right)^{t}, \quad\left(L^{*}\right)_{i j}=\left(L_{i j}\right)^{*}
$$


i.e., component-wise. If $\mathrm{O}$ is represented by the integral kernel $O\left(\mathbf{x}, \mathbf{x}^{\prime}\right)$, then the kernels of its transposed and hermitian-conjugated are given by

$$
O^{t}\left(\mathbf{x}, \mathbf{x}^{\prime}\right)=O\left(\mathbf{x}^{\prime}, \mathbf{x}\right), \quad O^{*}\left(\mathbf{x}, \mathbf{x}^{\prime}\right)=\overline{O\left(\mathbf{x}^{\prime}, \mathbf{x}\right)} .
$$

In particular, we have

$$
\begin{array}{cc}
\mathrm{U}^{t}=\mathrm{U}^{*}=\mathrm{U}^{-1}, & \Omega^{t}=\Omega^{*}=\Omega, \\
\mathrm{J}_{s}^{t}=\mathrm{J}_{s}, & \mathrm{~J}_{s}^{*}=\mathrm{J}_{-s}=\mathrm{J}_{s}^{-1} .
\end{array}
$$

In the Schrödinger representation we have $x^{t}=x, p^{t}=-p, x^{*}=x, p^{*}=p$ and hence (as seen from (6):

$$
\mathrm{e}_{s}^{t}=\mathrm{e}_{-s}, \quad \mathrm{f}_{s}^{t}=\mathrm{f}_{-s}, \quad \mathrm{k}_{s}^{t}=\mathrm{k}_{-s}^{-1},
$$

Properties of the transfer-matrices of models in question with respect to the transposition and hermitian-conjugation are described by the following statement.

Lemma 11. For the operations defined by (E.4) and (E.5) we have

$$
\begin{array}{rlrl}
\left(\mathrm{T}_{s}^{\mathrm{Xxz}}(u)\right)^{t} & =(-1)^{\mathrm{N}} \mathrm{T}_{-s}^{\mathrm{xxz}}(-u), & & \left(\mathrm{T}_{s}^{\mathrm{SG}}(u)\right)^{t}=\mathrm{T}_{-s}^{\mathrm{SG}}(-u) \\
\left(\mathrm{T}_{s}^{\mathrm{Xxz}}(u)\right)^{*}=\mathrm{T}_{s}^{\mathrm{Xxz}}(\bar{u}), & \left(\mathrm{T}_{s}^{\mathrm{SG}}(u)\right)^{*}=\mathrm{T}_{s}^{\mathrm{SG}}(\bar{u}),
\end{array}
$$

where $\mathrm{T}_{-s}(u) \equiv \mathrm{J}_{s} \mathrm{~T}_{s}(u) \mathrm{J}_{s}^{-1}$.

Proof. Taking into account (E.9), we observe that the L-matrices (10) and (33) satisfy

$$
\begin{aligned}
\left(L_{s}^{\mathrm{Xxz}}(u)\right)^{t} & =-\sigma_{3} e^{\pi b u \sigma_{3}} L_{-s}^{\mathrm{xxz}}(-u) e^{-\pi b u \sigma_{3}} \sigma_{3}, \\
\left(L_{s}^{\mathrm{SG}}(u)\right)^{t} & =\sigma_{3} L_{-s}^{\mathrm{SG}}(-u) \sigma_{3},
\end{aligned}
$$

Substitution of these relation into

$$
\mathrm{T}^{t}(u)=\operatorname{tr}\left(\left(L_{1}^{t}\right)^{T} \cdot\left(L_{2}^{t}\right)^{T} \cdot \ldots \cdot\left(L_{\mathrm{N}}^{t}\right)^{T}\right)=\operatorname{tr}\left(L_{\mathrm{N}}^{t} \cdot \ldots \cdot L_{2}^{t} \cdot L_{1}^{t}\right)
$$

yields $(\mathrm{E} .10)$. Relations $\left(\mathrm{E} .11\right.$ are derived analogously by noticing that we have $(L(u))^{*}=$ $\sigma_{3} L(\bar{u}) \sigma_{3}$ for both models in question.

A consequence of this Lemma is that it suffices to prove Theorem 2 only for $Q_{+}^{b}(u)$. Indeed, using (E.6) and (A.24), it is easy to conclude that

$$
\mathrm{Q}_{-}^{b}(u)=\left(D_{-s}(u)\right)^{\mathrm{N}}\left(\mathrm{Q}_{+}^{b}(\bar{u})\right)^{*} .
$$

Therefore relations (55-i)-(55-iii) for $\mathrm{Q}_{-}^{b}(u)$ then follow immediately if we take (E.11) into account. To check the Baxter equation (55-iv) for $Q_{-}^{b}(u)$, we take hermitian-conjugation of (55-iv) for $\mathrm{Q}_{+}^{b}(u)$, using (55-ii) and the property (E.10). After replacement of $\bar{u}$ by $u$ this yields for $\tilde{\mathrm{Q}}_{-}^{b}(u) \equiv\left(\mathrm{Q}_{+}^{b}(\bar{u})\right)^{*}$ the following equation

$$
\mathbf{T}^{b}(u) \cdot \tilde{\mathbf{Q}}_{-}^{b}(u)=(\overline{a(\bar{u})})^{\mathrm{N}} \tilde{\mathbf{Q}}_{-}^{b}(u+i b)+(\overline{d(\bar{u})})^{\mathrm{N}} \tilde{\mathbf{Q}}_{-}^{b}(u-i b) .
$$

Using relations A.21 and A.23, we observe that

$$
\overline{a(\bar{u})}=\frac{D_{-s}(u+i b)}{D_{-s}(u)} d(u), \quad \overline{d(\bar{u})}=\frac{D_{-s}(u-i b)}{D_{-s}(u)} a(u) .
$$

Whence we conclude that $\mathrm{Q}_{-}^{b}(u)$ defined by (E.14) satisfies 55-iv) (with the same coefficients $a(u), d(u)$ as $\mathrm{Q}_{+}^{b}(u)$ does). 


\section{E.2 Construction of Q-operators}

In order to construct the Q-operators explicitly let us consider the following general ansatz for the Q-operator:

$$
\mathrm{Q}(u)=\mathrm{Y}(u) \cdot \mathrm{Z} .
$$

We will prove Theorem 2 for $Q_{+}^{b}(u)$ in three steps: first constructing a suitable solution for $Y^{b}(u)$ by requiring the Baxter equation to hold, then determining the form of $Z^{b}$, and finally checking that the obtained Q-operator satisfies (55). The first step in this proof is based on the idea to find such a gauge transformation of the L-matrix that it becomes effectively upper-triangular. This approach was originally applied by Pasquier and Gaudin [PG] to the Toda chain. Our computation has many similarities with the modification of this approach developed in [De, DKM] for the non-compact XXX magnet.

Proposition 5. Let $\mathrm{T}^{\mathrm{b}}(u), b=\mathrm{xxz}, \mathrm{Sg}$ be the transfer-matrices corresponding to the L-matrices (10) and (33). Let $\mathrm{Y}^{b}(u)$ be defined in the Schrödinger representation by the kernel

$$
Y_{u}\left(\mathbf{x}, \mathbf{x}^{\prime}\right)=\prod_{r=1}^{\mathrm{N}} D_{\frac{1}{2}(u-\sigma)}\left(x_{r}-\varepsilon_{b} x_{r+1}^{\prime}\right) D_{-\frac{1}{2}(u+\sigma)}\left(x_{r}-x_{r}^{\prime}\right),
$$

where $\varepsilon_{\mathrm{xxz}}=1, \varepsilon_{\mathrm{SG}}=-1$. Then $\mathrm{Q}^{b}(u)$ of the form (E.17) satisfies the Baxter equation (55-iv) with coefficients $a(u), d(u)$ as specified in eq. 62 of Theorem 2

Proof. Let us introduce the gauge-transformed Lax operators (the transformation depends on the site number $r$ ):

$$
\begin{aligned}
\widetilde{L}_{r}^{b}(u) & =G_{r+1}^{b} \cdot L_{r}^{b}(u) \cdot\left(G_{r}^{b}\right)^{-1}, \quad G_{r}^{b}=\left(\begin{array}{cc}
1 & 0 \\
\rho_{r}^{\mathrm{b}} & 1
\end{array}\right), \\
\rho_{r}^{\mathrm{Xxz}} & =e^{\pi b\left(2 x_{r}^{\prime}-u\right)}, \quad \rho_{r}^{\mathrm{SG}}=e^{2 \pi b x_{r}^{\prime}} .
\end{aligned}
$$

The relevant matrix elements of the new Lax matrices are given by

$$
\begin{aligned}
\left(\widetilde{L}_{r}^{b}(u)\right)_{21}= & 4 \varkappa_{b}\left(\rho_{r} \rho_{r+1}\right)^{\frac{1}{2}} \\
& \times\left(\cosh \pi b\left(\mathrm{x}_{r}-\varepsilon_{b} x_{r+1}^{\prime}+\frac{1}{2}(\sigma-u)\right) \cosh \pi b\left(\mathrm{x}_{r}-x_{r}^{\prime}+\frac{1}{2}(\sigma+u)\right) \mathrm{k}_{r}\right. \\
& \left.-\cosh \pi b\left(\mathrm{x}_{r}-\varepsilon_{b} x_{r+1}^{\prime}-\frac{1}{2}(\sigma-u)\right) \cosh \pi b\left(\mathrm{x}_{r}-x_{r}^{\prime}-\frac{1}{2}(\sigma+u)\right) \mathrm{k}_{r}^{-1}\right), \\
\left(\widetilde{L}_{r}^{b}(u)\right)_{11}= & 2 \varkappa_{b} e^{\pi b\left(x_{r}^{\prime}-\varepsilon_{b} \mathrm{x}_{r}\right)}\left(e^{\frac{1}{2} \varepsilon_{b} \pi b(u-\sigma)} \cosh \pi b\left(\mathrm{x}_{r}-x_{r}^{\prime}+\frac{1}{2}(\sigma+u)\right) \mathrm{k}_{r}\right. \\
& \left.-e^{\frac{1}{2} \varepsilon_{b} \pi b(\sigma-u)} \cosh \pi b\left(\mathrm{x}_{r}-x_{r}^{\prime}-\frac{1}{2}(\sigma+u)\right) \mathrm{k}_{r}^{-1}\right) \\
\left(\widetilde{L}_{r}^{b}(u)\right)_{22}= & 2 \varkappa_{b} e^{\pi b\left(x_{r+1}^{\prime}-\mathrm{x}_{r}\right)}\left(e^{\frac{1}{2} \pi b(\sigma+u)} \cosh \pi b\left(\mathrm{x}_{r}-\varepsilon_{b} x_{r+1}^{\prime}+\frac{1}{2}(u-\sigma)\right) \mathrm{k}_{r}^{-1}\right. \\
& \left.-e^{-\frac{1}{2} \pi b(u+\sigma)} \cosh \pi b\left(\mathrm{x}_{r}-\varepsilon_{b} x_{r+1}^{\prime}+\frac{1}{2}(\sigma-u)\right) \mathrm{k}_{r}\right)
\end{aligned}
$$

where $\varkappa_{\mathrm{xxz}}=1, \varkappa_{\mathrm{SG}}=-i e^{-\pi b s}$.

In the Schrödinger representation, operators $\mathrm{k}_{r}, \mathrm{k}_{r}^{-1}$ act as shifts of $x_{r}$ by $\pm \frac{i}{2} b$. Using the functional relation (A.21), it is straightforward to apply E.21) to $Y_{u}^{b}\left(\mathbf{x}, \mathbf{x}^{\prime}\right)$ and verify that the condition

$$
\left(\widetilde{L}_{r}^{b}(u)\right)_{21} Y_{u}^{b}\left(\mathbf{x}, \mathbf{x}^{\prime}\right)=0
$$


is satisfied for all $\mathbf{x}^{\prime} \in \mathbb{R}^{\mathrm{N}}$. This implies that $\widetilde{L}_{r}^{b}(u)$ becomes upper triangular when acting on $Y_{u}^{b}\left(\mathbf{x}, \mathbf{x}^{\prime}\right)$ so that we can calculate the action of $\widetilde{\mathrm{M}}^{b}(u)$ on $Y_{u}^{b}\left(\mathbf{x}, \mathbf{x}^{\prime}\right)$ as

$$
\widetilde{\mathrm{M}}^{b}(u) Y_{u}^{b}\left(\mathbf{x}, \mathbf{x}^{\prime}\right)=\left(\begin{array}{cc}
\prod_{r=1}^{\mathrm{N}}\left(\widetilde{L}_{r}^{b}(u)\right)_{11} & * \\
0 & \prod_{r=1}^{\mathrm{N}}\left(\widetilde{L}_{r}^{b}(u)\right)_{22}
\end{array}\right) Y_{u}^{b}\left(\mathbf{x}, \mathbf{x}^{\prime}\right) .
$$

Hence, taking into account the periodicity condition, $G_{\mathrm{N}+1}^{b}=G_{1}^{b}$, we have

$$
\mathrm{T}^{b}(u) Y_{u}^{b}\left(\mathbf{x}, \mathbf{x}^{\prime}\right)=\left(\prod_{r=1}^{\mathrm{N}}\left(\widetilde{L}_{r}^{b}(u)\right)_{11}+\prod_{r=1}^{\mathrm{N}}\left(\widetilde{L}_{r}^{b}(u)\right)_{22}\right) Y_{u}^{b}\left(\mathbf{x}, \mathbf{x}^{\prime}\right) .
$$

Applying (E.22), E.23) to $Y_{u}^{b}\left(\mathbf{x}, \mathbf{x}^{\prime}\right)$ and using (E.24), we derive

$$
\begin{aligned}
& \left(\widetilde{L}_{r}^{b}(u)\right)_{11} Y_{u}^{b}\left(\mathbf{x}, \mathbf{x}^{\prime}\right)=2 \varepsilon_{b} \varkappa_{b} e^{\pi b\left(x_{r}^{\prime}-x_{r+1}^{\prime}\right)} \sinh \pi b(u-\sigma) Y_{u-i b}^{b}\left(\mathbf{x}, \mathbf{x}^{\prime}\right), \\
& \left(\widetilde{L}_{r}^{b}(u)\right)_{22} Y_{u}^{b}\left(\mathbf{x}, \mathbf{x}^{\prime}\right)=2 \varkappa_{b} e^{\pi b\left(x_{r+1}^{\prime}-x_{r}^{\prime}\right)} \sinh \pi b(u+\sigma) Y_{u+i b}^{b}\left(\mathbf{x}, \mathbf{x}^{\prime}\right) .
\end{aligned}
$$

Here we have used that $Y_{u}^{b}\left(\mathbf{x}, \mathbf{x}^{\prime}\right)=\prod_{r=1}^{\mathrm{N}} Y^{\mathrm{b}}\left(u, x_{r}\right)$, where each factor satisfies the relation

$$
\frac{Y^{b}\left(u, x_{r} \pm i \frac{b}{2}\right)}{Y^{b}\left(u \pm i b, x_{r}\right)}=\left(\frac{\cosh \pi b\left(x_{r}-x_{r}^{\prime}-\frac{1}{2}(\sigma+u)\right)}{\cosh \pi b\left(x_{r}-\varepsilon_{b} x_{r+1}^{\prime}+\frac{1}{2}(\sigma-u)\right)}\right)^{ \pm 1} .
$$

Combining (E.26) with (E.27), we obtain

$$
\begin{aligned}
\mathrm{T}^{b}(u) Y_{u}^{b}\left(\mathbf{x}, \mathbf{x}^{\prime}\right)= & \left(2 \varkappa_{b} \sinh \pi b(u+\sigma)\right)^{\mathrm{N}} Y_{u+i b}^{b}\left(\mathbf{x}, \mathbf{x}^{\prime}\right) \\
& +\left(2 \varepsilon_{b} \varkappa_{b} \sinh \pi b(u-\sigma)\right)^{\mathrm{N}} Y_{u-i b}^{b}\left(\mathbf{x}, \mathbf{x}^{\prime}\right),
\end{aligned}
$$

which implies that the Baxter equation (55-iv) holds with the coefficients $a(u), d(u)$ as specified in eq. 62.

The possible form of $\mathrm{Z}$ can be found from the requirement that [55-iii) holds.

Proposition 6. Let $\mathrm{Y}^{b}(u)$ be chosen as in Proposition 5 Then the commutativity condition

$$
\mathrm{Q}^{b}(u) \mathrm{T}^{b}(u)=\mathrm{T}^{b}(u) \mathrm{Q}^{b}(u)
$$

holds for $\mathrm{Q}^{b}(u)$ of the form (E.17) provided that the corresponding operator $Z^{b}$ satisfies the following relation

$$
\mathrm{Z}^{b} \mathrm{~T}_{s}^{b}(u)=\mathrm{T}_{-s}^{b}(u) \mathrm{Z}^{b}
$$

where the $-s$ is understood in the sense specified in Lemma 17

Proof. In order to treat both models in a uniform way, let us introduce the operator

$$
\Omega^{b}=\left\{\begin{array}{ll}
1, & b=\mathrm{xxz} \\
\Omega, & b=\mathrm{SG}
\end{array},\right.
$$

where the parity operator was defined in (E.1). Then, using the explicit expressions (E.18) and taking A.22 into account, it is easy to verify that (the subscript $\mathbf{x}$ or $\mathrm{x}^{\prime}$ of an operator specifies the argument on which it acts)

$$
\Omega_{\mathbf{x}}^{b} Y_{u}^{b}\left(\mathbf{x}, \mathbf{x}^{\prime}\right)=\Omega_{\mathbf{x}^{\prime}}^{b} Y_{u}^{b}\left(\mathbf{x}, \mathbf{x}^{\prime}\right)=\mathrm{U}_{\mathbf{x}}^{-1} Y_{-u}^{b}\left(\mathbf{x}^{\prime}, \mathbf{x}\right) .
$$


Now we derive

$$
\begin{aligned}
& \mathrm{T}_{s ; \mathbf{x}}^{b}(u) Y_{u}^{b}\left(\mathbf{x}, \mathbf{x}^{\prime}\right) \stackrel{\text { E.29 }}{=}(a(u))^{\mathrm{N}} Y_{u-i b}^{b}\left(\mathbf{x}, \mathbf{x}^{\prime}\right)+(d(u))^{\mathrm{N}} Y_{u+i b}^{b}\left(\mathbf{x}, \mathbf{x}^{\prime}\right) \\
& \stackrel{\text { E.33 }}{=}\left(-\varepsilon_{b}\right)^{\mathrm{N}} \Omega_{\mathbf{x}}^{b} \mathrm{U}_{\mathbf{x}}^{-1}\left((d(-u))^{\mathrm{N}} Y_{-u+i b}^{b}\left(\mathbf{x}^{\prime}, \mathbf{x}\right)+(a(-u))^{\mathrm{N}} Y_{-u-i b}^{b}\left(\mathbf{x}^{\prime}, \mathbf{x}\right)\right) \\
& \stackrel{\text { E.29 }}{=}\left(-\varepsilon_{b}\right)^{\mathrm{N}} \Omega_{\mathbf{x}}^{b} \mathrm{U}_{\mathbf{x}}^{-1} \mathrm{~T}_{s ; \mathbf{x}^{\prime}}^{b}(-u) Y_{-u}^{b}\left(\mathbf{x}^{\prime}, \mathbf{x}\right) \stackrel{\text { E.33 }}{=}\left(-\varepsilon_{b}\right)^{\mathrm{N}} \mathrm{T}_{s ; \mathbf{x}^{\prime}}^{b}(-u) Y_{u}^{b}\left(\mathbf{x}, \mathbf{x}^{\prime}\right) \\
& =\left(-\varepsilon_{b}\right)^{\mathrm{N}} Y_{u}^{b}\left(\mathbf{x}, \mathbf{x}^{\prime}\right)\left(\mathrm{T}_{s ; \mathbf{x}^{\prime}}^{\mathrm{SG}}(-u)\right)^{t} \stackrel{\mathrm{E} .10}{=} Y_{u}^{b}\left(\mathbf{x}, \mathbf{x}^{\prime}\right) \mathrm{T}_{-s ; \mathbf{x}^{\prime}}^{b}(u) .
\end{aligned}
$$

Thus, we verified that $\mathrm{T}_{s}^{b}(u) \mathrm{Y}(u)=\mathrm{Y}(u) \mathrm{T}_{-s}^{b}(u)$, which is equivalent to E.30 if relation E.31 is satisfied.

Proposition 6 implies that we can choose

$$
\mathrm{Z}^{b}=\mathrm{J}_{s}
$$

where $J_{s}$ was defined in $E .2$. Then we compute the integral kernel of $Q_{+}^{b}(u)$ :

$$
\begin{aligned}
& Q_{+; u}^{b}\left(\mathbf{x}, \mathbf{x}^{\prime}\right)=\left(w_{b}\left(i \frac{Q}{2}-2 s\right)\right)^{\mathrm{N}} \int_{\mathbb{R}^{\mathrm{N}}} d z_{1} \ldots d z_{\mathrm{N}} \prod_{r=1}^{\mathrm{N}} D_{\frac{u-\sigma}{2}}\left(z_{r}-\varepsilon_{b} x_{r-1}\right) \\
& \times D_{-\frac{u+\sigma}{2}}\left(z_{r}-x_{r}\right) D_{\bar{\sigma}}\left(z_{r}-x_{r}^{\prime}\right) \\
&=\left(D_{-s}(u)\right)^{\mathrm{N}} \prod_{r=1}^{\mathrm{N}} D_{\frac{\bar{\sigma}-u}{2}}\left(x_{r}-x_{r}^{\prime}\right) D_{\frac{\bar{\sigma}+u}{2}}\left(x_{r-1}-\varepsilon_{b} x_{r}^{\prime}\right) D_{-s}\left(x_{r}-\varepsilon_{b} x_{r-1}\right) .
\end{aligned}
$$

Equivalence of (E.35) and (E.36) is due to the identity A.34.

Remark 9. The Baxter equation (55-iv) along with the self-commutativity (55-ii) relation, which will be proven below, imply that $\left(\right.$ E.30) extends to commutativity of $\mathrm{Q}_{+}^{b}(u)$ with $\mathrm{T}^{b}(v)$ for those values of $v$, where $\mathrm{Q}_{+}^{b}(v)$ is invertible.

\section{E.3 Proof of commutativity relations}

To complete the proof of Theorem 2 we have to establish relations (55-i) and [55-ii).

Lemma 12. Let $\mathrm{Y}^{b}(u)$ be chosen as in Proposition 5 Then the following identities hold

$$
\begin{aligned}
& \left(\mathrm{Y}^{b}(\bar{u})\right)^{*} \cdot \mathrm{Y}^{b}(v)=\left(D_{s}(u) D_{-s}(v)\right)^{\mathrm{N}}\left(\mathrm{Y}^{b}(\bar{v})\right)^{*} \cdot \mathrm{Y}^{b}(u), \\
& \mathrm{Y}^{b}(u) \cdot\left(\mathrm{Y}^{b}(\bar{v})\right)^{*}=\left(D_{s}(v) D_{-s}(u)\right)^{\mathrm{N}} \mathrm{Y}^{b}(v) \cdot\left(\mathrm{Y}^{b}(\bar{u})\right)^{*} .
\end{aligned}
$$

Proof. These identities are just particular cases of the integral identity A.35. Indeed, let us denote $\alpha_{u} \equiv \frac{1}{2}(u-\sigma), \beta_{u} \equiv-\frac{1}{2}(u+\sigma)$. We will also use the notation $\alpha^{\star} \equiv-\frac{i}{2} Q-\alpha$. Let us consider the operator $\mathrm{V}^{b}(u, v)=\left(\mathrm{Y}^{b}(\bar{u})\right)^{*} \cdot \mathrm{Y}^{b}(v)$. Its kernel is given by

$$
\begin{aligned}
V_{u, v}^{b}\left(\mathbf{x}, \mathbf{x}^{\prime}\right)=\int_{\mathbb{R}^{\mathrm{N}}} d z_{1} \ldots d z_{\mathrm{N}} \prod_{r=1}^{\mathrm{N}} D_{\alpha_{u}^{\star}}\left(z_{r}-\varepsilon_{b} x_{r+1}\right) D_{\beta_{u}^{\star}}\left(z_{r}-x_{r}\right) \\
\times D_{\alpha_{v}}\left(z_{r}-\varepsilon_{\mathrm{b}} x_{r+1}^{\prime}\right) D_{\beta_{v}}\left(z_{r}-x_{r}^{\prime}\right),
\end{aligned}
$$


Now we can apply identity A.35 choosing $\alpha=\alpha_{u}^{\star}, \beta=\alpha_{v}, \gamma=\beta_{u}^{\star}, \omega=\beta_{v}$, and $u=\varepsilon_{b} x_{r+1}$, $v=\varepsilon_{b} x_{r+1}^{\prime}, w=x_{r}, z=x_{r}^{\prime}$. This yields

$$
\begin{aligned}
V_{u, v}^{b}\left(\mathbf{x}, \mathbf{x}^{\prime}\right)= & \left(A\left(\alpha_{u}^{\star}, \alpha_{v}, \beta_{u}^{\star}, \beta_{v}\right)\right)^{\mathrm{N}} \prod_{r=1}^{\mathrm{N}} D_{\frac{v-u}{2}}\left(\varepsilon_{b}\left(x_{r+1}-x_{r+1}^{\prime}\right)\right) D_{\frac{u-v}{2}}\left(x_{r}-x_{r}^{\prime}\right) \\
& \times \int_{\mathbb{R}^{\mathrm{N}}} d z_{1} \ldots d z_{\mathrm{N}} \prod_{r=1}^{\mathrm{N}} D_{\alpha_{u}}\left(z_{r}-\varepsilon_{b} x_{r+1}^{\prime}\right) D_{\beta_{u}}\left(z_{r}-x_{r}^{\prime}\right) \\
& \times D_{\alpha_{v}^{\star}}\left(z_{r}-\varepsilon_{b} x_{r+1}\right) D_{\beta_{v}^{\star}}\left(z_{r}-x_{r}\right) \\
= & \left(D_{s}(u) D_{-s}(v)\right)^{\mathrm{N}} V_{v, u}^{b}\left(\mathbf{x}, \mathbf{x}^{\prime}\right) .
\end{aligned}
$$

Here we used A.22, the definition A.28 of the function $A\left(\alpha_{1}, \alpha_{2}, \ldots\right)$, and took into account the periodic boundary conditions.

Identity (E.38) can be proven absolutely analogously.

Proposition 7. The operators $Q_{+}^{b}(u)$ and $Q_{-}^{b}(u)$ with the kernels given in Theorem 2 by eqs. (60) and (61), respectively, satisfy the following commutativity and exchange relations

$$
\begin{gathered}
Q_{+}^{b}(u) Q_{+}^{b}(v)=Q_{+}^{b}(v) Q_{+}^{b}(u), \quad Q_{-}^{b}(u) Q_{-}^{b}(v)=Q_{-}^{b}(v) Q_{-}^{b}(u), \\
Q_{+}^{b}(u) Q_{-}^{b}(v)=Q_{-}^{b}(u) Q_{+}^{b}(v)=Q_{+}^{b}(v) Q_{-}^{b}(u)=Q_{-}^{b}(v) Q_{+}^{b}(u) .
\end{gathered}
$$

for all $u, v \in \mathbb{C}$.

Proof. Observe that, using E.6 and A.24, the equality of E.35 and E.36 can be written in the following operator form:

$$
\mathrm{Q}_{+}^{b}(u)=\mathrm{Y}^{b}(u) \cdot \mathrm{J}_{s}=\left(D_{-s}(u)\right)^{\mathrm{N}}\left(\mathrm{X}_{s}^{b}\right)^{-1} \cdot \Omega^{b} \cdot \mathrm{U}^{-1} \cdot\left(\mathrm{Y}^{b}(\bar{u})\right)^{*},
$$

where we used the notation $(E .32$ and introduced

$$
\mathrm{X}_{s}^{b}=\prod_{r=1}^{\mathrm{N}} D_{s}\left(\mathrm{x}_{r}-\varepsilon_{b} \mathrm{x}_{r-1}\right) .
$$

Using Lemma 12, we can write down the product of two such Q-operators as follows

$$
\begin{aligned}
& \mathrm{Q}_{+}^{b}(u) \mathrm{Q}_{+}^{b}(v)=\left(D_{-s}(u)\right)^{\mathrm{N}}\left(\mathrm{X}_{s}^{b}\right)^{-1} \cdot \Omega^{b} \cdot \mathrm{U}^{-1} \cdot\left(\mathrm{Y}^{b}(\bar{u})\right)^{*} \cdot \mathrm{Y}^{b}(v) \cdot \mathrm{J}_{s} \\
& \stackrel{\mathrm{E} .37}{=}\left(D_{-s}(v)\right)^{\mathrm{N}}\left(\mathrm{X}_{s}^{b}\right)^{-1} \cdot \Omega^{b} \cdot \mathrm{U}^{-1} \cdot\left(\mathrm{Y}^{b}(\bar{v})\right)^{*} \cdot \mathrm{Y}^{b}(u) \cdot \mathrm{J}_{s}=\mathrm{Q}_{+}^{b}(v) \mathrm{Q}_{+}^{b}(u) .
\end{aligned}
$$

This proves the first relation in E.41) and hence [55-ii) for $Q_{+}^{b}(u)$. As was explained in Subsection E.1 relation (55-ii) for $Q_{-}^{b}(u)$ (i.e., the second relation in (E.41) follows then as a consequence of the relation (E.14) between $Q_{+}^{b}(u)$ and $Q_{-}^{b}(u)$. By the same token, relation (55-i) is equivalent to (E.42). To prove the latter relation, we substitute (E.43) into (E.14) and use (E.7)E.8). This yields the operator $Q_{-}^{b}(u)$ in the following form:

$$
\mathrm{Q}_{-}^{b}(u)=\left(D_{-s}(u)\right)^{\mathrm{N}} \mathrm{J}_{s}^{-1} \cdot\left(\mathrm{Y}^{b}(\bar{u})\right)^{*}=\mathrm{Y}^{b}(u) \cdot \mathrm{U} \cdot \Omega^{b} \cdot \mathrm{X}_{s}^{b} .
$$

As seen from (E.43) and E.46, the two expressions on the 1.h.s. of E.42) are just two ways to write down $\left(D_{-s}(v)\right)^{\mathrm{N}} \mathrm{Y}^{b}(u) \cdot\left(\mathrm{Y}^{\mathrm{b}}(\bar{v})\right)^{*}$. Analogously, the two expressions on the r.h.s. of E.42 are two ways to write down $\left(D_{-s}(u)\right)^{\mathrm{N}} \mathrm{Y}^{\mathrm{b}}(v) \cdot\left(\mathrm{Y}^{\mathrm{b}}(\bar{u})\right)^{*}$. The middle equality in (E.42) is due to the identity (E.38) in Lemma 12. 


\section{References}

[Ba] E.W. Barnes: Theory of the double gamma function, Phil. Trans. Roy. Soc. A196 (1901) 265-388

[BL] V. Brazhnikov and S. Lukyanov: Angular quantization and form factors in massive integrable models Nucl. Phys. B512 (1998) 616-636

[BD] A.G. Bytsko and A. Doikou: Thermodynamics and conformal properties of XXZ chains with alternating spins, J. Phys. A37 (2004) 4465-4492

[BT] A. Bytsko and J. Teschner: R-operator, co-product and Haar-measure for the modular double of $\mathcal{U}_{q}(\mathfrak{s l}(2, \mathbb{R}))$, Commun. Math. Phys. 240 (2003) 171-196

[De] S.E. Derkachov: Baxter's Q-operator for the homogeneous XXX spin chain, J. Phys. A32 (1999) $5299-5316$

[DKK] S.E. Derkachov, D. Karakhanyan, and R. Kirschner: Baxter Q-operators of the XXZ chain and R-matrix factorization, Nucl. Phys. B738 (2006) 368-390

[DKM] S.E. Derkachov, G.P. Korchemsky, and A. N. Manashov: Noncompact Heisenberg spin magnets from high-energy QCD. I. Baxter Q-operator and separation of variables, Nucl. Phys. B617 (2001) 375-440; Separation of variables for the quantum $S L(2, \mathbb{R})$ spin chain, JHEP 0307 (2003) 047

[F1] L.D. Faddeev: How algebraic Bethe ansatz works for integrable model. In: Symétries quantiques (North-Holland, 1998), 149-219 |hep-th/9605187|

[F2] L.D. Faddeev: Discrete Heisenberg-Weyl group and modular group, Lett. Math. Phys. 34 (1995) 249-254

[F3] L.D. Faddeev: Modular double of a quantum group, Math. Phys. Stud. 21 (2000) 149-156

[FK2] L.D. Faddeev and R.M. Kashaev: Quantum dilogarithm, Mod. Phys. Lett. A9 (1994) 427-434

[FKV] L.D. Faddeev, R.M. Kashaev, and A.Yu. Volkov: Strongly coupled quantum discrete Liouville theory. I: Algebraic approach and duality, Commun. Math. Phys. 219 (2001) 199-219

[FK1] L.D. Faddeev and G.P. Korchemsky: High energy QCD as a completely integrable model, Phys. Lett. B342 (1995) 311-322

[FST] L.D. Faddeev, E.K. Sklyanin, and L.A. Takhtajan: Quantum inverse problem method. I, Theor. Math. Phys. 40 (1979) 688-706

[FTT] L.D. Faddeev, V.O. Tarasov, and L.A. Takhtajan: Local Hamiltonians for integrable quantum models on a lattice, Theor. Math. Phys. 57 (1983) 1059-1073

[FT] L.D. Faddeev and O. Tirkkonen: Connections of the Liouville model and XXZ spin chain, Nucl. Phys. B453 (1995) 647-669

[FV] L.D. Faddeev and A.Yu. Volkov: Yang-Baxterization of the quantum dilogarithm, Zapiski nauch. semin. POMI 224 (1995) 146-154 [Engl. transl.: J. Math. Sci. 88 (1998) 202-207]

[FMS] A. Fring, G. Mussardo, and P. Simonetti: Form-factors for integrable Lagrangian field theories, the sinh-Gordon theory, Nucl. Phys. B393 (1993) 413-441

[IK] A.G. Izergin and V.E. Korepin: Lattice versions of quantum field theory models in two dimensions, Nucl. Phys. B205 (1982) 401-413

[Ji] M. Jimbo: A q-difference analogue of $U(g l(N+1))$ and the Yang-Baxter equations, Lett. Math. Phys. 10 (1985) 63-69

[K1] R.M. Kashaev: The non-compact quantum dilogarithm and the Baxter equations, J. Stat. Phys. 102 (2001) 923-936

[K2] R.M. Kashaev: The quantum dilogarithm and Dehn twists in quantum Teichmüller theory, In: Integrable structures of exactly solvable two-dimensional models of quantum field theory (Kiev, 2000), 211-221 (NATO Sci.Ser.II Math.Phys.Chem., 35, Kluwer Acad. Publ., Dordrecht, 2001)

[KL] S. Kharchev, D. Lebedev: Integral representation for the eigenfunctions of quantum periodic Toda chain, Lett. Math. Phys. 50 (1999) 53-77 
[KLS] S. Kharchev, D. Lebedev, and M. Semenov-Tian-Shansky: Unitary representations of $\mathcal{U}_{q}(\mathfrak{s t}(2, \mathbb{R}))$, the modular double, and the multiparticle q-deformed Toda chains, Commun. Math. Phys. 225 (2002) 573-609

[KM] M. Kirch and A.N. Manashov: Noncompact $S L(2, \mathbb{R})$ spin chain, JHEP 0406 (2004) 035

[KBI] V. Korepin, N. Bogoliubov, and A. Izergin: Quantum inverse scattering method and correlation functions (Cambridge U. Press, 1993)

[KMu] A. Koubek and G. Mussardo: On the operator content of the sinh-Gordon model, Phys. Lett. B311 (1993) 193-201

[Le] G. Lechner: An existence proof for interacting quantum field theories with a factorising S-matrix, math-ph/0601022

[Li] L.N. Lipatov: High energy asymptotics of multi-colour QCD and exactly solvable lattice models, JETP Lett. 59 (1994) 596-599

[Lu] S. Lukyanov: Finite temperature expectation values of local fields in the sinh-Gordon model, Nucl. Phys. B612 (2001) 391-412

[PG] V. Pasquier and M. Gaudin: The periodic Toda chain and a matrix generalization of the Bessel function, J. Phys. A25 (1992) 5243-5252

[PT1] B. Ponsot and J. Teschner: Liouville bootstrap via harmonic analysis on a non-compact quantum group, hep-th/9911110

[PT2] B. Ponsot and J. Teschner: Clebsch-Gordan and Racah-Wigner coefficients for a continuous series of representations of $\mathcal{U}_{q}(\mathfrak{s l}(2, \mathbb{R}))$, Commun. Math. Phys. 224 (2001) 613-655

$[\mathrm{Ru}]$ S.N.M. Ruijsenaars: First order analytic difference equations and integrable quantum systems, J. Math. Phys. 38 (1997) 1069-1146

[S] K. Schmüdgen: Integrable operator representations of $\mathbb{R}_{q}^{2}, X_{q, \gamma}$ and $\mathrm{SL}_{q}(2, \mathbb{R})$, Commun. Math. Phys. 159 (1994) 217-237

[Sh] T. Shintani: On a Kronecker limit formula for real quadratic fields, J. Fac. Sci. Univ. Tokyo Sect. 1A Math. 24 (1977) 167-199

[S1] E.K. Sklyanin: Exact quantization of the sinh-Gordon model, Nucl. Phys. B326 (1989) 719-736

[S2] E.K. Sklyanin: The quantum Toda chain, Lect. Notes Phys. 226 (1985) 196-233

[S3] E.K. Sklyanin: Quantum inverse scattering method. Selected topics. In: Quantum groups and quantum integrable systems (World Scientific, 1992) 63-97 |hep-th/9211111|; Separation of variables new trends, Prog. Theor. Phys. Suppl. 118 (1995) 35-60

[Sm1] F.A. Smirnov: Quasi-classical study of form factors in finite volume. In: L.D. Faddeev's seminar on mathematical physics (AMS Transl. Ser. 2, 201, AMS, Providence, RI, 2000) 283-307 |hep-th/9802132|

[Sm2] F.A. Smirnov: Baxter equations and deformation of abelian differentials, Int. J. Mod. Phys. A19S2 (2004) 396-417.

[Ta] V.O. Tarasov: Irreducible monodromy matrices for the $R$ matrix of the XXZ model and local lattice quantum Hamiltonians, Theor. Math. Phys. 63 (1985) 440-454

[T1] J. Teschner: On structure constants and fusion rules in the $S L(2, \mathbb{C}) / S U(2)-W Z N W$ model, Nucl. Phys. B546 (1999) 390-422; Operator product expansion and factorization in the $\mathrm{H}_{3}^{+}-W Z N W$ model, Nucl. Phys. B571 (2000) 555-582; Crossing symmetry in the $H_{3}^{+}$WZNW model, Phys. Lett. B521 (2001) 127-132

[T2] J. Teschner: Liouville theory revisited, Class. Quant. Grav. 18 (2001) R153-R222; A lecture on the Liouville vertex operators, Int. J. Mod. Phys. A19S2 (2004) 436-458

[Ti] E.C. Titchmarsh: The theory of functions, 2nd ed. (Oxford Univ. Press, Oxford, 1975)

[VG] S.N. Vergeles and V.M. Gryanik: Two-dimensional quantum field theories which admit exact solutions, Sov. J. Nucl. Phys. 23 (1976) 704-709 
[V1] A.Yu. Volkov: Quantum Volterra model, Phys. Lett. A167 (1992) 345-355

[V2] A.Yu. Volkov: Noncommutative hypergeometry, Commun. Math. Phys. 258 (2005) 257-273

[Wo] S.L. Woronowicz: Quantum exponential function, Rev. Math. Phys. 12 (2000) 873-920

[Za] Al. Zamolodchikov: On the thermodynamic Bethe Ansatz equation in sinh-Gordon model, hep-th/0005181

[ZZ] A.B. Zamolodchikov and Al.B. Zamolodchikov: Structure constants and conformal bootstrap in Liouville field theory, Nucl. Phys. B477 (1996) 577-605 